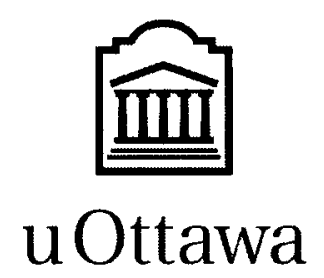

L'Université canadrenne' Canada's university 
FACULTÉ DES ÉTUDES SUPÉRIEURES ET POSTOCTORALES

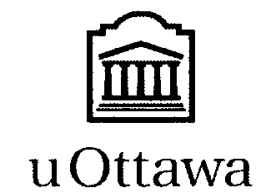

uOttawa

Unimexsite canddienne

C'dnadd's unversity

\section{FACULTY OF GRADUATE AND POSDOCTORAL STUDIES}

Stéphane Lozier

AUTEUR DE LA THESE / AUTHORO OF THESIS

M.Sc. (Mathematics) GRADE/DEGREE

Faculty of Science - Department of Mathematics

FACULLTE, ECOOLE, DEPARTEMMENT / FACULTY, SCHOOOL, DEPARTMENENT

On Simultaneous Approximation to a Real Number and its Cube by Rational Numbers

TITRE DE LA THÉSE/TITLE OF THESIS

D. Roy

DIRECTEUA (DIRECTAICE) DE LA THESE/ THESIS SUPERVISOR

CO-DIRECTEUA (CO-DIRECTRICE) DE LA THESE / THESIS CO-SUPERVISOR

A. Sebbar

S. Alaca

Gary W. Slater

Le Doyen de la Faculté des études supérieures et postdoctorales / Dean of the Faculty of Graduate and Postdoctoral Studies 


\title{
On simultaneous approximation to a real number and its cube by rational numbers
}

\author{
Stéphane Lozier
}

Thesis Submitted to the Faculty of Graduate and Postdoctoral Studies In partial fulfilment of the requiremonts for the degree of Master of Science in Mathematics ${ }^{1}$

Department of Mathematics and Statistics

Faculty of Srience

University of Ottawa.

(C) Stéphane Lozier, Ottawa, Canada, 2010

\footnotetext{
${ }^{1}$ The M.Sc. program is a joint program with Carleton University, administered by the OttawaCarleton Institute of Mathematics and Statistics
} 
Library and Archives Canada

Published Heritage Branch

395 Wellington Street Ottawa ON K1A ON4 Canada
Bibliothèque et

Archives Canada

Direction du

Patrimoine de l'édition

395 , rue Wellington

Ottawa ON K1A ON4

Canada
Your file Votre référence

ISBN: 978-0-494-73781-1

Our file Notre référence

ISBN: 978-0-494-73781-1
NOTICE:

The author has granted a nonexclusive license allowing Library and Archives Canada to reproduce, publish, archive, preserve, conserve, communicate to the public by telecommunication or on the Internet, loan, distribute and sell theses worldwide, for commercial or noncommercial purposes, in microform, paper, electronic and/or any other formats.

The author retains copyright ownership and moral rights in this thesis. Neither the thesis nor substantial extracts from it may be printed or otherwise reproduced without the author's permission.
AVIS:

L'auteur a accordé une licence non exclusive permettant à la Bibliothèque et Archives Canada de reproduire, publier, archiver, sauvegarder, conserver, transmettre au public par télécommunication ou par l'Internet, prêter, distribuer et vendre des thèses partout dans le monde, à des fins commerciales ou autres, sur support microforme, papier, électronique et/ou autres formats.

L'auteur conserve la propriété du droit d'auteur et des droits moraux qui protège cette thèse. Ni la thèse ni des extraits substantiels de celle-ci ne doivent être imprimés ou autrement reproduits sans son autorisation.
In compliance with the Canadian Privacy Act some supporting forms may have been removed from this thesis.

While these forms may be included in the document page count, their removal does not represent any loss of content from the thesis.
Conformément à la loi canadienne sur la protection de la vie privée, quelques formulaires secondaires ont été enlevés de cette thèse.

Bien que ces formulaires aient inclus dans la pagination, il n'y aura aucun contenu manquant. 


\section{Abstract}

One of the fundamental problems in Diophantine approximation is approximation to real numbers by algebraic numbers of bounded degree. In 1969. II. Davenport and W. M. Schmidt developed a new method to approach the problem. This method combines a result on simultaneous approximation to successive powers of a real number $\xi$ with geometry of numbers For now, the only case where the estimates are optimal is the case of two consecutive powers. Davenport and Schmidt show that if a real number $\xi$ is such that $1 . \xi, \xi^{2}$ are linearly independent over $\mathbb{Q}$. then the exponent of smultaneous approximation to $\xi$ and $\xi^{2}$ by rational numbers with the same denominator is at most $(\sqrt{5}-1) / 2=0.618 \ldots$ the inverse of the Golden ratio. In this thesis, we consider the case of a number and its cube. Our main result is that if a real number $\xi$ is such that $1, \xi, \xi^{3}$ are linearly independent over $\mathbb{Q}$, then the exponent of simultaneous approximation to $\xi$ and $\xi^{3}$ by rational numbers with the same denominator is at most $5 / 7=0.714 \ldots$. As corollaries, we deduce a result on approximation by algebraic numbers and a version of Gel'fond's lemma for polynomials of the form $a+b T+c T^{3}$. 


\section{Résumé}

Un des problèmes fondamentaux en approximation diophantionne est celu de l'approximation des nombres réels par des nombres algébriques de degré borné. En 1969. H. Davenport et W. M. Schmidt ont développé une nouvelle méthode pour approcher ce problème. Elle combine un résultat d’approximation simultané de puissances consécutives d un nombre réel $\xi$ avec des méthodes de géométric des nombres. Pour l'instant le seul cas où les estimations sont optimales est celui de deux puissances consćcutives. Davenport et Schmidt ont démontré que si un nombre réel $\xi$ est tel que $1 . \xi . \xi^{2}$ sont linćrairement indépendants sur $\mathbb{Q}$. alors l'exposant d'approximation simultané de $\xi$ et de $\xi^{2}$ par des nombres rationnels de même dénominateur est an plus $(\sqrt{5}-1) / 2=0.618 \ldots$ soit linverse du nombre d'or. Dans ce mémoire, nous considérons le cas d'un nombre et de son cube. Notre résultat principal est que si un nombre réel $\xi$ est tel que $1, \xi, \xi^{3}$ sont linéairement indépendants sur $\mathbb{Q}$. alors l'exposant d'approximation simultané do $\xi$ et de $\xi^{3}$ par des nombres ratiomels de même dénominateur est au plus $5 / 7=0.714 \ldots$ Nous en déduisons des corollaires sous la forme d'un résultat d'approximation par des nombres algébriques ct d'une version du lemme de Gel'fond concernant les polynômes de la forme $a+b T+c T^{3}$. 


\section{Contents}

Abstract

Résumé

1 Introduction 1

1.1 History ........................... 1

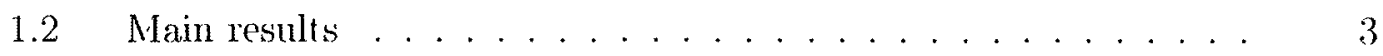

1.3 Outline of the thesis and methodology .......... 6

1.4 Notation ....................... 8

2 Minimal points and sequences of minimal points 9

2.1 Minimal points . . . . . . . . . . . . . . . 9

2.2 Sequences of minimal points .............. 11

2.3 Some key lemmas . . . . . . . . . . . . . . 14

3 The function $\varphi$ and a first estimate $\quad 19$

4 A family of planes in $\mathbb{R}^{3} \quad 23$

5 The function $\Phi$ and the point $\psi \quad 27$

6 Intermediate points and large systems of inequalities 32 
7 The functions $f$ and $g \quad 43$

8 The point $S$ and estimates for $g \quad 49$

8.1 Various new points . . . . . . . . . . . . 49

8.2 The point $S \ldots \ldots \ldots \ldots \ldots$

8.3 New estimates for $g \ldots \ldots \ldots . \ldots . \ldots . \ldots 5$

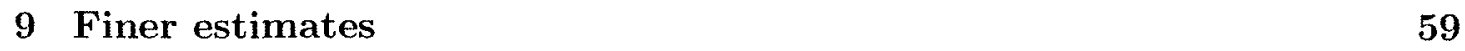

9.1 Finer estimates for $p \ldots \ldots \ldots$. . . . . . . . . . . 60

9.2 Finer estimates for $g \ldots \ldots \ldots 2 \ldots \ldots \ldots$

9.3 A further consequence of the vanishing of $f \ldots 64$

9.4 Finer estimates for $S \ldots \ldots \ldots 5$

10 The function $k$ and the last estimate $\quad 69$

$\begin{array}{ll}\text { A Finding new relations } & 74\end{array}$

$\begin{array}{ll}\text { B Polynomials } & 77\end{array}$ 


\section{Chapter 1}

\section{Introduction}

\subsection{History}

In therr 1969 paper [4], II. Davenport and W. M. Schmidt established the following result, where

$$
\gamma=\frac{1+\sqrt{5}}{2}=1+\frac{1}{\gamma}=1.618
$$

denotes the golden ratio.

Theorem 1.1.1 (Davenport-Schmidt) Suppose that $\xi \in \mathbb{R}$ is nether rational nor a quadratic irrational. Then there are arbitrarly large values of $X$ such that the inequalities

$$
\left|x_{0}\right| \leq X, \quad\left|x_{0} \xi-x_{1}\right| \leq c_{1} X^{-1 / \gamma}, \quad\left|x_{0} \xi^{2}-x_{2}\right| \leq c_{1} X^{-1 / \gamma}
$$

where $c_{1}$ is a suitable positive number depending only on $\xi$, have no solution in integers $x_{0} \cdot x_{1} \cdot x_{2}$ not all zero.

If $\left(x_{0}, x_{1}, x_{2}\right) \in \mathbb{Z}^{3}$ is a non-zero integer solution of the inequalities (1.1.1) of Theorem 1.1.1 for some large value of $X$, then we must have $x_{0} \neq 0$ and we obtain

$$
\max \left\{\left|\xi-\frac{x_{1}}{x_{0}}\right|,\left|\xi^{2}-\frac{x_{2}}{x_{0}}\right|\right\} \leq c_{1} \frac{X^{-1 / \gamma}}{\left|x_{0}\right|} \leq c_{1}\left|x_{0}\right|^{-\gamma}
$$


which means that the pair $\left(x_{1} / x_{0}, x_{2} / x_{0}\right)$ is a good approximation to $\left(\xi, \xi^{2}\right)$ by rational numbers with the same denominator.

By an argument based on geometry of numbers, Davenport and Schmidt deduce the following corollary where the height $H(\alpha)$ of an algebraic number $\alpha$ is defined as the height of its minimal polynomial in $\mathbb{Z}[T]$, the height $H(P)$ of a polynomial $P \in \mathbb{R}[T]$ being defined as the largest absolute value of its coefficients.

Corollary 1.1.2 (Davenport-Schmidt) Let $\xi$ be as in Theorem 1.1.1. Then there are infinitely many algebraic integers $\alpha$ of dcgree at most 3 over $\mathbb{Q}$ which satisfy

$$
|\xi-\alpha| \leq c_{2} H(\alpha)^{-\gamma-1}
$$

for a suitable constant $c_{2}=c_{2}(\xi)>0$.

The same method also leads to a similar result of approximation by algebraic numbers of degree at most 2 :

Corollary 1.1.3 Let $\xi$ be as in Theorem 1.1.1. Then there are infinitely many algebraic numbers $\alpha$ of degree at most 2 over $\mathbb{Q}$ which satisfy

$$
|\xi-\alpha| \leq c_{3} H(\alpha)^{-\gamma-1}
$$

for a suitable constant $c_{3}=c_{3}(\xi)>0$.

However, although it is known that the estimates in Theorem 1.1.1 and in Corollary 1.1 .2 are the best possible (see [7] and [8]), this is not the case for Corollary 1.1.3. Indeed, Davenport and Schmidt showed in [3] that in (1.1.2) the exponent $-\gamma-1 \approx-2.618$ can be replaced by -3 , in which case the estimate is optimal.

In another direction, Jarník's transference principle ('Theorem 1 of [5]) also allows one to restate Theorem 1.1.1 in the following dual form:

Corollary 1.1.4 Let $\xi$ be as in Theorem 1.1.1. Then, there are arbitrarty large values of $Y$ such that the inequalities

$$
\left|y_{1}\right| \leq Y, \quad\left|y_{2}\right| \leq Y, \quad\left|y_{0}+y_{1} \xi+y_{2} \xi^{2}\right| \leq c_{4} Y^{-\gamma-1}
$$


where $c_{4}=c_{4}(\xi)>0$ is a suitable positive number depending only on $\xi$, have no solution in integers $y_{0}, y_{1}, y_{2}$ not all zero.

The latter corollary can be viewed as a version of Gel'fond's lemma in degree 2. A sharper result, with an explicit value for the constant $c_{4}$ is given in [1]:

Theorem 1.1.5 (Arbour-Roy) Let $\xi \in \mathbb{R}$. Suppose that for each sufficiently large value of $Y$ there exists a non-zero polynomial $P \in \mathbb{Z}[T]$ of degree $\leq 2$ satisfying

$$
H(P) \leq Y \quad \text { and } \quad|P(\xi)| \leq \frac{1}{4} Y^{-\gamma-1} .
$$

Then $\xi$ is algebraic over $\mathbb{Q}$ of degree $\leq 2$.

\subsection{Main results}

In this thesis. we are looking at simultaneous approximation to a real number and to its cube by rational numbers with the same denominator. Our main result is the following:

Theorem 1.2.1 Let $\xi \in \mathbb{R}$ be such that $1, \xi, \xi^{3}$ are linearly independent over $\mathbb{Q}$, and let $\lambda>5 / 7$. Then there are arbitrarily large values of $X$ for which the inequalities

$$
\left|x_{0}\right| \leq X . \quad\left|x_{0} \xi-x_{1}\right| \leq X^{-\lambda}, \quad\left|x_{0} \xi^{3}-x_{2}\right| \leq X^{-\lambda}
$$

admit no non-zero solution $\left(x_{0}, x_{1}, x_{2}\right) \in \mathbb{Z}^{3}$.

We don't know if this statement remains true for $\lambda=5 / 7$ even at the expense of replacing the upper bound $X^{-\lambda}$ by $c_{5} X^{-\lambda}$ for a suitably small positive constant $c_{5}$.

$\Lambda$ pplying the method of Davenport and Schmidt to the above result, we deduce the following analog of Corollary 1.1.3:

Corollary 1.2.2 Let $\xi$ and $\lambda$ be as in Theorem 1.2.1. Then there are infinitely many alyebraic numbers $\alpha$ which are roots of polynomials of the form $a T^{3}+b T+c$ with 
$a, b, c \in \mathbb{Z}$ and which satisfy

$$
|\xi-\alpha| \leq X^{-1-1 / \lambda}
$$

We do not present the details of the proof of this corollary in this thesis since there are abundant examples of such in the literature, starting from the original work of Davenport and Schmidt in Section 2 of [4] (see for example Section 2 of [9] or Chapter 2 of [11]).

Using Jarnik's transference principle, Theorem 1.2.1 admits the following equivalent form, analogous to Theorem 1.1.5:

Corollary 1.2.3 Let $\xi \in \mathbb{R}$ and let $\tau>7 / 2$. Suppose that, for each sufficiently large $Y$, there exists a non-zero polynomial $P(T) \in \mathbb{Z} T^{3}+\mathbb{Z} T+\mathbb{Z}$ satisfying

$$
H(P) \leq Y \quad \text { and } \quad|P(\xi)| \leq Y^{-\tau}
$$

Then $\xi$ is a root of a non-zero polynomial in $\mathbb{Z} T^{3}+\mathbb{Z} T+\mathbb{Z}$.

We give a direct proof a weaker result in Appendix B (corresponding to the measure found in Chapter 3).

The rest of the thesis is mainly concerned with the proof of Theorem 1.2.1. We proceed in several steps. In order to describe them. it is useful to make the following definitions:

Definition 1.2.4 Let $\xi . \eta \in \mathbb{R}$. We say that a real number $\mu$ is a measure of simultaneous approximation to $(1, \xi, \eta)$ if there exists a constant $c>0$, and arbitrarily large values of $X$, such that the inequalities

$$
\left|x_{0}\right| \leq X, \quad\left|x_{0} \xi-x_{1}\right| \leq c X^{-\mu}, \quad\left|x_{0} \eta-x_{2}\right| \leq c X^{-\mu}
$$

have no non-zero solution $\left(x_{0}, x_{1}, x_{2}\right) \in \mathbb{Z}^{3}$. When this holds, we say that $c$ is a constant associated to the measure $\mu$. 
Definition 1.2.5 Let $\xi, \eta \in \mathbb{R}$. We say that a real number $\lambda$ is an exponent of simultaneous approximation to $(1, \xi, \eta)$ if there exssts a constant $c>0$ such that the inequalities

$$
\left|x_{0}\right| \leq X . \quad\left|x_{0} \xi-x_{1}\right| \leq c X^{-\lambda}, \quad\left|x_{0} \eta-x_{2}\right| \leq c X^{-\lambda}
$$

have a non-zero solution $\left(x_{0}, x_{1}, x_{2}\right) \in \mathbb{Z}^{3}$ for any sufficuently large $X$. In this case, we say that $\mathrm{C}$ is a constant associated to the exponent $\lambda$.

From these definitions. Theorem 1.1 .1 can be restated by saying that $1 / \gamma$ is a measure of approximation to $\left(1, \xi, \xi^{2}\right)$ for any $\xi \in \mathbb{R}$ such that $1 . \xi, \xi^{2}$ are linearly independent over $\mathbb{Q}$. Similarly, Theorem 1.2.1 says that each real $\lambda>5 / 7$ is a measure of approximation to $\left(1, \xi, \xi^{3}\right)$ for anv $\xi \in \mathbb{R}$ such that $1, \xi . \xi^{3}$ are linearly independent over $\mathbb{Q}$.

Moreover, by Minkowski’s Linear Foums Theorem [10. Chapter II, Theorem 2C], we have:

Theorem 1.2.6 Let $\xi, \eta \in \mathbb{R}$. For every $X>1$ the inequalities

$$
\left|x_{0}\right| \leq X . \quad\left|x_{0} \xi-x_{1}\right|<X^{-1 / 2} . \quad\left|x_{0} \eta-x_{2}\right|<X^{-1 / 2}
$$

have a non-zero solution $\left(x_{0}, x_{1}, x_{2}\right) \in \mathbb{Z}^{3}$.

This shows that, for any $\xi . \eta \in \mathbb{R}$. the number $\lambda=1 / 2$ is an exponent of simultaneous approximation to $(1, \xi, \eta)$ and $c=1$ is a constant associated with this exponent. We also note the following properties which follow immediately from the definitions:

- If $\mu \in \mathbb{R}$ is a measure of approximation to a triple $(1, \xi, \eta)$, then any real number $\mu^{\prime}$ with $\mu \leq \mu^{\prime}$ is also a measure of approximation to the same triple.

- If $\lambda \in \mathbb{R}$ is an exponent of approximation to $(1, \xi, \eta)$, then any real number $\lambda^{\prime}$ with $\lambda^{\prime} \leq \lambda$ is another such exponent of approximation. 
- If $\mu$ is a measure of approximation to $(1, \xi, \eta)$ and if $\lambda$ is an exponent of approximation to the same triple, then $\lambda \leq \mu$.

- If $\lambda$ is not a measure of approximation to $(1, \xi . \eta)$, then $\lambda$ is an exponent of approximation to the same triple and. as such, its associated constant can be taken to be arbitrarily small.

- If $\mu$ is not an exponent of approximation to $(1 . \xi . \eta)$. then $\mu$ is a measure of approximation to the same triple and. as such, its associated constant can be taken to be arbitrarily large.

In view of these remarks, Theorem 1.2 .1 can be restated by saying that, if $\lambda$ is an exponent of approximation to $\left(1, \xi . \xi^{3}\right)$ for some $\xi \in \mathbb{R}$ such that $1 . \xi . \xi^{3}$ are linearly independent over $\mathbb{Q}$. then $\lambda \leq 5 / 7$.

The reason why we rest rict to triples $(1, \xi, \eta)$ where $1, \xi, \eta$ are linear ly independent over $\mathbb{Q}$ is that ot helwise, Dirichlet's Theorem [10. Chapter I, Theorem 1A] implies that $\lambda=1$ is an exponent of approximation to $(1, \xi, \eta)$.

\subsection{Outline of the thesis and methodology}

The chapters of this thesis represent the different stages which lead me finally to Theorem 1.2.1. In each one. new tools or new methods are introduced to improve the upper bound on any exponent of approximation $\lambda$ to a triple $\left(1, \xi, \xi^{3}\right)$ with $\mathbb{Q}$-linearly 
independent entries. I find successively:

$$
\begin{array}{llrl}
\lambda & \leq \sqrt{3}-1=0.732 \ldots & & \text { (Chapter 3) } \\
\lambda & \leq 0.7277 \ldots & & \text { (Chapter 5) } \\
\lambda & \leq 8 / 11=0.7272 \ldots & & \text { (Chapter 6) } \\
\lambda & \leq 0.7252 \ldots & & \text { (Chapter } 7) \\
\lambda & \leq 0.7221 \ldots & & \text { (Chapter } 8) \\
\lambda & \leq(9+\sqrt{15}) / 18=0.7151 \ldots & & \text { (Chapter 9) } \\
\lambda \leq 5 / 7=0.7142 \ldots & & \text { (Chapter 10). }
\end{array}
$$

All results rely on the construction of a so-called sequence of minimal points for $\left(1, \xi, \xi^{3}\right)$ following ideas of Davemport and Schmidt in Section 3 of $[4]$. This is a serpence of integer points $\mathbf{x}_{\imath}=\left(x_{\imath, 0}, x_{i, 1}, x_{\imath, 2}\right) \in \mathbb{Z}^{3}$ which is canonically attached to the triple $\left(1, \xi, \xi^{3}\right)$ and from which one can deduce whether or not a given $\lambda>0$ is an exponent of approximation to $\left(1, \xi, \xi^{3}\right)$. Upon putting

$$
\begin{aligned}
& X_{i}=\max \left\{\left|x_{i, 0}\right| \cdot\left|x_{i, 1}\right| \cdot\left|x_{i, 2}\right|\right\}, \\
& L_{i}=\max \left\{\left|x_{i, 0} \xi-x_{i, 1}\right|,\left|x_{i, 0} \xi^{3}-x_{i, 2}\right|\right\},
\end{aligned}
$$

this condition is simply that

$$
L_{i} \leq c X_{i+1}^{-\lambda}
$$

for some constant $c>0$ (by construction, we also have $X_{1}<X_{2}<X_{3}<\ldots$ and $\left.L_{1}>L_{2}>L_{3}>\ldots\right)$.

The main tool is the construction of polynomials with specific properties. If

$$
P\left(\mathbf{u}_{0}, \mathbf{u}_{1}, \ldots, \mathbf{u}_{k}\right) \in \mathbb{Z}\left[\mathbf{u}_{0}, \mathbf{u}_{1}, \ldots, \mathbf{u}_{k}\right]
$$

is a multi-homogeneous polynomial in triples of indeterminates $\mathbf{u}_{j}=\left(u_{j, 0}, u_{j, 1}, u_{j, 2}\right)$, upper bound estimates for

$$
\left|P\left(\mathbf{x}_{i}, \mathbf{x}_{i+1}, \ldots, \mathbf{x}_{1+k}\right)\right|
$$


can be given in terms of $X_{\imath} \ldots X_{\imath+k}, L_{\imath} \ldots, L_{\imath+k}$ (see $\Lambda$ ppendix A). When $P$ is suitably constructed. then, depending on the value of $\lambda$, this upper bound tends to 0 as $\imath \rightarrow \infty$. and therefore the integers (1.3.1) are all zero for each sufficiently large $\imath$ (see Proposition A.1).

Some of these polynomials are relatively easy to find, like the polynomial $\varphi$ from Chapter 3. The polvnomials or polynomial points $w, f, g, S, k$ that appear in the subsequent chapteis came from a computer search. Each new polynomial formd in this way presents a challenge. First. one needs a compact way of writing it (some of them involve many monomials). Then, one has to understand how the polynomials are related with one another, and finally one has to understand what their vanishing means. When we can show that some polynomial $P$ as above does not vanish at $\left(\mathbf{x}_{2} \ldots \mathbf{x}_{1+k}\right)$ for infinitely many values of 1 , then we get an upper bound for $\lambda$. For example. the upper bound $\lambda \leq \sqrt{3}-1=0.732 \ldots$ is obtained in Chapter 3 by showing that for $\lambda>2 / 3$ we have $x_{\imath, 0}^{2} x_{\imath, 2}-x_{\imath .1}^{3} \neq 0$ for each $i$ sufficiently large. The subsequent improvements are more challenging and we let the reader discover them as he goes through the thesis. We simply mention that the final estimate $\lambda \leq 5 / 7$ follows by observing first that if $\lambda>5 / 7$ then $f$ must vanish at certain pairs of points. From this, we deduce that $k$ cannot vanish at related triples of points and this leads to a contradiction.

\subsection{Notation}

Notation 1.4.1 Throughout the text, for fixed real numbers $\xi$ and $\eta$. we will use the notation $A \ll B$ or $B \gg A$. where $A$ and $B$ are non-negative variable real numbers, to mean $A \leq c B$ for some constant $c>0$ depending only on $\xi$ and $\eta$. We will also write $A \asymp B$ if $A \ll B \ll A$. 


\section{Chapter 2}

\section{Minimal points and sequences of minimal points}

In their proof of Theorem 1.1.1, Davenport and Schmidt begin by defining a sequence of minimal points associated to a triple of the form $\left(1, \xi, \xi^{2}\right)$. In this chapter we work in greater generality by considering triples of the form $(1, \xi, \eta)$. The notion of minimal points that we attach to such a triple differs slightly from the definition of Davenport and Schmidt when $\eta=\xi^{2}$, but it is easy to adapt most of their arguments to our setting.

\subsection{Minimal points}

Definition 2.1.1 Let $\xi, \eta \in \mathbb{R}$. For each point $\mathrm{x}=\left(x_{0}, x_{1}, x_{2}\right) \in \mathbb{R}^{3}$, we define the norm and proximity functions:

$$
\|\mathbf{x}\|_{\infty}:=\max \left\{\left|x_{0}\right| .\left|x_{1}\right|,\left|x_{2}\right|\right\}, \quad L_{\xi, \eta}(\mathbf{x}):=\max \left\{\left|x_{0} \xi-x_{1}\right|,\left|x_{0} \eta-x_{2}\right|\right\} .
$$

We usually urite $\|\mathbf{x}\|:=\|\mathbf{x}\|_{\infty}$ and $L(\mathbf{x}):=L_{\xi, \eta}(\mathbf{x})$.

Throughout this section, we fix $\xi, \eta \in \mathbb{R} \backslash \mathbb{Q}$. Then, for any non-zero point $\mathrm{x} \in \mathbb{Z}^{3}$, we have $L(\mathbf{x})>0$. 
Definition 2.1.2 For each real $X \geq 1$, consider the set, of points $\mathrm{x} \in \mathbb{Z}^{3}$ with

$$
1 \leq\|\mathbf{x}\| \leq X, \quad L(\mathbf{x}) \leq 1
$$

A minimal point corresponding to $\mathrm{X}$ is a point $\mathbf{x}$ of thas set for which $L(\mathbf{x})$ is minimal.

Note. that since the set defined by (2.1.1) is finite and non-empty (e.g. it contains $(0,1,1)$ ), for any real $X \geq 1$, there exists a minimal point corresponding to $X$.

Lemma 2.1.3 If $\mathrm{x}=\left(x_{0}, x_{1}, x_{2}\right)$ is minimal point (for some real number $X \geq 1$ ), then $\mathbf{x}$ is primitive, i.e. we have $\operatorname{gcd}\left(x_{0}, x_{1}, x_{2}\right)=1$.

Proof: Indeed, if $a=\operatorname{gcd}\left(x_{0}, x_{1}, x_{2}\right)>1$ then $\mathbf{x}=a \mathbf{x}^{\prime}$ for some point $\mathbf{x}^{\prime} \in \mathbb{Z}^{3}$. This implies that $\left\|\mathbf{x}^{\prime}\right\|<\|\mathbf{x}\|$ and $L\left(\mathbf{x}^{\prime}\right)=\frac{1}{a} L(\mathbf{x})<L(\mathbf{x})$, so $\mathbf{x}$ is not minimal.

Lemma 2.1.4 Let $\tilde{\varepsilon}>0$. Then, for each $X$ sufficiently large, a minimal point $\mathbf{x}$ corresponding to $X$ satisfies $L(\mathbf{x}) \leq \varepsilon$.

Proof: By Theorem 1.2.6, there exists a non-zero $\mathbf{y} \in \mathbb{Z}^{3}$ with $L(\mathbf{y}) \leq \min \{1, \varepsilon\}$. Choose $X \geq\|\mathrm{y}\|$ and let $\mathrm{x}$ be a minimal point corresponding to $X$, then since $\mathrm{y}$ is a solution of the inequalities (2.1.1), we have that $L(\mathbf{x}) \leq L(\mathbf{y}) \leq \varepsilon$.

Lemma 2.1.5 Let $\mathrm{x}=\left(x_{0}, x_{1}, x_{2}\right) \in \mathbb{Z}^{3}$ be a minimal point corresponding to some $X \geq 1$. If $X$ is sufficiently large, then $\mathbf{x}$ has non-zero coordinates and satisfies

$$
\|\mathbf{x}\| \asymp\left|x_{i}\right|
$$

for $i=0,1,2$. 
Proof: We first note that. for each $X \geq 1$, a minimal point $\mathbf{x}=\left(x_{0}, x_{1} \cdot x_{2}\right)$ corresponding to $X$ satisfies

$$
\begin{aligned}
& \left|x_{0} \xi\right|-1 \leq\left|x_{1}\right| \leq\left|x_{0} \xi\right|+1 \\
& \left|x_{0} \eta\right|-1 \leq\left|x_{2}\right| \leq\left|x_{0} \eta\right|+1
\end{aligned}
$$

If such a minimal point $\mathbf{x}$ satisfies $\left|x_{0}\right|<2 / \min \{1 .|\xi|,|\eta|\}$, then, looking at the right-hand side of the above inequalities, we have $\|\mathbf{x}\| \ll 1$. Since there are a finitc number of points which satisfy $\|\mathbf{x}\| \ll 1$, this means that $L(\mathbf{x}) \asymp 1$. But by Lemma 2.1.4, taking $X$ sufficiently large we can always find a minimal point $\mathbf{x}^{\prime}$ conresponding to $X$ with $L\left(\mathbf{x}^{\prime}\right)$ as small as we want. So, for $X$ sufficiently large, we have that $\left|x_{0}\right| \geq 2 / \min \{1 \cdot|\xi| \cdot|\eta|\}$. Then, from the above inequalities we find that the coordinates of $\mathrm{x}$ are non-zero with $\left|x_{0}\right| \asymp\left|x_{1}\right|$ and $\left|x_{0}\right| \asymp\left|x_{2}\right|$.

\subsection{Sequences of minimal points}

From now on. we will suppose that $\xi . \eta \in \mathbb{R}$ are such that $1 . \xi . \eta$ are linearly independent ove1 $\mathbb{Q}$. We begin with a lemma which will be used to constuuct secunences of minimal points.

Lemma 2.2.1 Let $\xi . \eta \in \mathbb{R}$ be such that $1, \xi, \eta$ are lmearly independent over $\mathbb{Q}$. For any $X \geq 1+\max \{|\xi|,|\eta|\}$, there are exactly two minimal points corresponding to $X$. If $\mathbf{x}$ is one of them. then the other is $-\mathbf{x}$.

Proof: $\quad$ Fix some $X \geq 1+\max \{|\xi| \cdot|\eta|\}$. and a minimal point $\mathbf{x}=\left(x_{0}, x_{1}, x_{2}\right)$ colresponding to $X$.

We first show that $L(\mathbf{x})<1 / 2$ and deduce that $x_{0} \neq 0$. Indeed, since $(1,[\xi],[\eta])$ is a solution of the inequalities (2.1.1) of Definition 2.1.2. where $[\xi]$ and $[\eta]$ denote the 
int egers nearest to $\xi$ and $\eta$. respectively, we get $L(\mathbf{x}) \leq \max \{|\xi-[\xi]|,|\eta-[\eta]|\}<1 / 2$. Now, suppose on the contrary that $x_{0}=0$. This gives $1 \leq\|\mathbf{x}\|=\max \left\{\left|x_{1}\right|,\left|x_{2}\right|\right\}=$ $L(\mathbf{x})$, but since $L(\mathbf{x})<1 / 2$ this is impossible. So. we must have $x_{0} \neq 0$.

Now suppose that $\mathrm{x}^{\prime}=\left(x_{0}^{\prime}, x_{1}^{\prime}, x_{2}^{\prime}\right)$ is another minimal point for $X$. It satisfies $L(\mathbf{x})=L\left(\mathbf{x}^{\prime}\right)$. Then we have 4 possible cases.

In the first case we have $L(\mathbf{x})=\left|x_{0} \xi-x_{1}\right|$ and $L\left(\mathbf{x}^{\prime}\right)=\left|x_{0}^{\prime} \eta-x_{2}^{\prime}\right|$. This implies $x_{0} \xi-x_{1}= \pm\left(x_{0}^{\prime} \eta-x_{2}^{\prime}\right) \Rightarrow \mp x_{0}^{\prime} \eta+x_{0} \xi-\left(x_{1} \mp x_{2}^{\prime}\right)=0 \Rightarrow x_{0}=x_{0}^{\prime}=0, x_{1}= \pm x_{2}^{\prime}$

which is impossible since $x_{0} \neq 0$. The second case, when $L(\mathbf{x})=\left|x_{0} \eta-x_{2}\right|$ and $L\left(\mathbf{x}^{\prime}\right)=\left|x_{0}^{\prime} \xi-x_{1}^{\prime}\right|$. leads similarly to a contradiction.

In the third case, $L(\mathbf{x})=\left|x_{0} \xi-x_{1}\right|$ and $L\left(\mathbf{x}^{\prime}\right)=\left|x_{0}^{\prime} \xi-x_{1}^{\prime}\right|$. so we have

$$
x_{0} \xi-x_{1}= \pm\left(x_{0}^{\prime} \xi-x_{1}^{\prime}\right) \Rightarrow\left(x_{0} \mp x_{0}^{\prime}\right) \xi-\left(x_{1} \mp x_{1}^{\prime}\right)=0 \Rightarrow x_{0}= \pm x_{0}^{\prime}, x_{1}= \pm x_{1}^{\prime}
$$

and this implies

$\left|-x_{2} \pm x_{2}^{\prime}\right|=\left|x_{0} \eta-x_{2} \mp\left(x_{0}^{\prime} \eta-x_{2}^{\prime}\right)\right| \leq\left|x_{0} \eta-x_{2}\right|+\left|x_{0}^{\prime} \eta-x_{2}^{\prime}\right| \leq 2 L(\mathbf{x})<1 \Rightarrow x_{2}= \pm x_{2}^{\prime}$.

Thus $\mathbf{x}= \pm \mathbf{x}^{\prime}$. Finally, in the fourth case we have $L(\mathbf{x})=\left|x_{0} \eta-x_{2}\right|$ and $L\left(\mathbf{x}^{\prime}\right)=$ $\left|x_{0}^{\prime} \eta-x_{2}^{\prime}\right|$ and by a similar argument we again find that $\mathbf{x}= \pm \mathbf{x}^{\prime}$.

Note that if $\mathrm{x}$ is a minimal point (for some real number $X \geq 1$ ). then it is minimal for $\|\mathbf{x}\|$. So, on the basis of Lemma 2.2.1, we can construct recursively a secpuence of minimal points $\left(x_{1}\right)_{\imath \geq 1} \subseteq \mathbb{Z}^{3}$ which will satisfy the following definition.

Definition 2.2.2 Let $\xi, \eta \in \mathbb{R}$ be such that $1, \xi, \eta$ are lincarly indcpendent over $\mathbb{Q}$. We define a sequence of minimal points associated to $(1, \xi, \eta)$ to be a sequence of minimal point.s $\left(\mathbf{x}_{1}\right)_{1 \geq 1} \subseteq \mathbb{Z}^{3}$ of strictly increasing norms with the following property:

if $\mathbf{x}$ is a minimal point satısfyıng $\left\|\mathbf{x}_{\imath}\right\| \leq\|\mathbf{x}\|<\left\|\mathbf{x}_{1+1}\right\|$ then $\mathbf{x}= \pm \mathbf{x}_{\imath}$. 
Equivalently we could define such a sequence using the following property:

if $\mathbf{x}$ is a mmimal point satisfying $L\left(\mathbf{x}_{1+1}\right)<L(\mathbf{x}) \leq L\left(\mathbf{x}_{1}\right)$. then $\mathbf{x}= \pm \mathbf{x}_{1}$.

Note that any two sequences of minimal points associated to $(1, \xi, \eta)$ differ only by the choice of their first element and a choice of sign factors. In particular, if $\left(\mathrm{x}_{\imath}\right)_{1 \geq 1}$ is a sequence of minimal points, then $\left(\epsilon_{\imath} \mathbf{x}_{\imath}\right)_{\imath \geq r}$ is also a sequence of minimal points for any $r \geq 1$ and any choice of $\epsilon_{\imath} \in\{ \pm 1\}$. Thus if a sequence of minimal points satisfies a certain property for each $\imath$ sufficiently large. then by omitting its first elements we obtain a secuence of minimal points which obeys this property for each $\imath \geq 1$.

Notation 2.2.3 If $\left(x_{\imath}\right)_{l \geq 1}$ is a sequence of minimal points associated to $(1, \xi . \eta)$. we usually write

$$
\mathbf{x}_{\imath}=\left(x_{\imath, 0} \cdot x_{\imath, 1}, x_{r, 2}\right) . \quad X_{\imath}:=\left\|\mathbf{x}_{\imath}\right\| \quad \text { and } \quad L_{\imath}:=L\left(\mathbf{x}_{\imath}\right)
$$

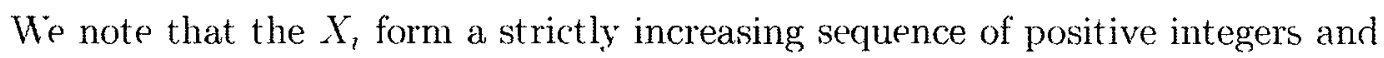
that the $L_{1}$ form a strictly decreasing sequence of positive real numbers.

Moreover, for any two points $\mathbf{x}_{1}, \mathbf{x}_{j}$ of the sequence with $\imath \neq j$, becdlse they are primitive points with $\mathbf{x}_{\imath} \neq \mathbf{x}_{j}$ (see Lemma 2.1.4), we have that $\mathbf{x}_{\imath}$ and $\mathbf{x}_{1}$ are linearly independent.

Let $\left(\mathbf{x}_{\imath}\right)_{1 \geq 1}$ be a sequence of minimal points associated to $(1, \xi, \eta)$. Note that if $x_{\imath .0}=0$, then $(0 . \pm 1 . \pm 1)$ are four different points that are minimal points corresponding to $\left\|\mathrm{x}_{\imath}\right\|$, in contradiction with Definition 2.2.2. So, for all $l \geq 1$ we have $x_{2,0} \neq 0$. Because of this, we must have $L\left(\mathbf{x}_{1}\right)<1$. Noreover, for an appropiate choice of $\epsilon_{1}, \epsilon_{2} \in\{0, \pm 1\}$, the point $\mathbf{x}=\left(x_{1,0} \cdot x_{1,1}+\epsilon_{1} \cdot x_{1,2}+\epsilon_{2}\right)$ satisfies $L(\mathbf{x})<1 / 2$ and $\|\mathbf{x}\| \leq\left\|\mathbf{x}_{1}\right\|+1 \leq\left\|\mathbf{x}_{2}\right\|$. So, for all $\iota \geq 2$ we have $L\left(\mathbf{x}_{\imath}\right)<1 / 2$. Finally, we note that, for every $r$ sufficicntly large and appropriate choices of $\epsilon_{\imath} \in\{ \pm 1\}$ the points $\epsilon_{1} \mathbf{x}_{\imath}$ remain in the same octant as $(1, \xi, \eta)$. 
Lemma 2.2.4 Let $\xi, \eta \in \mathbb{R}$ be such that $1, \xi, \eta$ are linearly independent over $\mathbb{Q}$. Let $\left(\mathbf{x}_{i}\right)_{1 \geq 1}$ be a sequence of minimal points associated to (1. $\left.\xi . \eta\right)$. Then for each choice of $j \geq i \geq 2$ we have $\left|x_{j, k}\right| \geq\left|x_{\imath, k}\right|$ for $k=0,1,2$.

Proof: $\quad$ For $i \geq 2$ we have $L\left(\mathbf{x}_{j}\right) \leq L\left(\mathbf{x}_{i}\right)<1 / 2$. If $\left|x_{j, 0}\right| \geq\left|x_{i, 0}\right|$ then we have $\left|x_{3,1}\right|+1 / 2>\left|x_{, 00} \xi\right| \geq\left|x_{i, 0} \xi\right|>\left|x_{i, 1}\right|-1 / 2$ which gives $\left|x_{j, 1}\right| \geq\left|x_{\imath, 1}\right|$ since $\mathbf{x}_{2}$ and $\mathbf{x}_{j}$ are integer points. Similarly we find $\left|x_{j, 2}\right| \geq\left|x_{i, 2}\right|$.

There are three possible cases: $\max \{1,|\xi|,|\eta|\}=1, \max \{1,|\xi|,|\eta|\}=|\xi|$ or $\max \{1,|\xi|,|\eta|\}=|\eta|$

In the first case we get $\left|x_{j, 0}\right|>\left|x_{j, 0} \xi\right|>\left|x_{3.1}\right|-1 / 2$ which implies that $\left|x_{j, 0}\right| \geq$ $\left|x_{j, 1}\right|$ and similarly we obtain $\left|x_{, .0}\right| \geq\left|x_{j, 2}\right|$. Thus $\left\|\mathbf{x}_{j}\right\|=\left|x_{j, 0}\right|$. Since $\left\|\mathbf{x}_{j}\right\| \geq\left\|\mathbf{x}_{i}\right\|$ we conclude that $\left|x_{3,0}\right| \geq\left|x_{\imath, 0}\right|$ and we are done.

In the second case we get $\left|x_{j, 1}\right|+1 / 2>\left|x_{j, 0} \xi\right|>\left|x_{,, 0}\right|$ and this gives $\left|x_{j, 1}\right| \geq\left|x_{j, 0}\right|$. We also have $\left|x_{j, 1}\right|+1 / 2>\left|x_{j, 0} \xi\right|>\left|x_{j, 0} \eta\right|>\left|x_{j, 2}\right|-1 / 2$ and we get $\left|x_{j, 1}\right| \geq\left|x_{j, 2}\right|$. Thus $\left\|\mathbf{x}_{j}\right\|=\left|x_{j .1}\right|$. Since $\left\|\mathbf{x}_{j}\right\| \geq\left\|\mathbf{x}_{r}\right\|$ we now obtain $\left|x_{j, 0} \xi\right|+1 / 2>\left|x_{j .1}\right| \geq\left|x_{i .1}\right|>$ $\left|x_{i, 0} \xi\right|-1 / 2$ which implies $1>1 /|\xi|>\left|x_{i, 0}\right|-\left|x_{\jmath, 0}\right|$ and again we are done.

The third case leads similarly to $\left|x_{j, 0}\right| \geq\left|x_{i, 0}\right|$.

\subsection{Some key lemmas}

The next series of Lemmas provide deeper results that we will use throughout this thesis. The first of these could be described as the main property of a sequence of minimal points. It lies at the basis of almost all further considerations.

Lemma 2.3.1 Let $\xi, \eta \in \mathbb{R}$ be such that $1, \xi, \eta$ are linearly independent over $\mathbb{Q}$. Let $\left(\mathbf{x}_{i}\right)_{i \geq 1}$ be a sequence of minimal points associated to (1. $\left.\xi . \eta\right)$. Suppose that $\lambda>0$ is an exponent of approximation to $(1, \xi, \eta)$ and that $c>0$ is a constant associated to 
this exponent. Then. for each i sufficiently large, we have

$$
L_{\imath} \leq c c_{0}^{\lambda} X_{\imath+1}^{-\lambda}
$$

where $c_{0}=2 \max \{1,|\xi|,|\eta|\}$.

Proof: Fix some $i \geq 1$ and put $X=2\left(X_{i+1}-1\right) / c_{0}$. If $i$ is sufficiently large, the hypotheses imply the existence of a non-zero solution $\mathbf{x}=\left(x_{0}, x_{1}, x_{2}\right) \in \mathbb{Z}^{3}$ of the inequalitios of Definition 1.2.5. For such a point $\mathbf{x}$, we have

$$
\begin{gathered}
\left|x_{0}\right| \leq X \leq X_{i+1}-1<X_{1+1} \\
\left|x_{1}\right| \leq\left|x_{0} \xi\right|+c X^{-\lambda}<|\xi| X+1 \leq X_{i+1} \\
\left|x_{2}\right| \leq\left|x_{0} \eta\right|+c X^{-\lambda}<|\eta| X+1 \leq X_{i+1}
\end{gathered}
$$

Thus $\|\mathrm{x}\|<X_{\imath+1}$, and so we obtain

$$
L_{\imath}=L\left(\mathbf{x}_{i}\right) \leq L(\mathbf{x}) \leq c X^{-\lambda}=c c_{0}^{\lambda}\left(2\left(X_{i+1}-1\right)\right)^{-\lambda} \leq c c_{0}^{\lambda} X_{i+1}^{-\lambda}
$$

where the last estimate uses $X_{1-1} \geq X_{2} \geq 2$ and so $2\left(X_{i+1}-1\right) \geq X_{i+1}$.

Lemma 2.3.2 Let $\xi, \eta \in \mathbb{R}$ be such that $1, \xi, \eta$ are linearly indcpendent over $\mathbb{Q}$. Let $\left(\mathbf{x}_{i}\right)_{i \geq 1}$ be a sequence of minimal points associated to $(1, \xi, \eta)$. Suppose that $\mathbf{x}_{2-1}, \mathbf{x}_{\imath}, \mathbf{x}_{i+1}$ are linearly indepcndent. Then

$$
1 \ll X_{\imath+1} L_{i-1} L_{i}
$$

Proof: Since $\mathbf{x}_{i-1}, \mathbf{x}_{i}, \mathbf{x}_{i+1}$ are linearly independent, the determinant of these three points is a non-zero integer and so its absolute value is $\geq 1$. We now find an upper bound for the determinant:

$$
\left\|\begin{array}{ccc}
x_{i-1,0} & x_{i-1,1} & x_{\imath-1,2} \\
x_{i, 0} & x_{i, 1} & x_{i, 2} \\
x_{i+1,0} & x_{i+1,1} & x_{i+1,2}
\end{array}\right\|=\left\|\begin{array}{ccc}
x_{i-1,0} & x_{i-1,0} \xi-x_{i-1,1} & x_{i-1,0} \eta-x_{i-1,2} \\
x_{i, 0} & x_{i, 0} \xi-x_{i, 1} & x_{\imath, 0} \eta-x_{i, 2} \\
x_{i+1,0} & x_{i+1,0} \xi-x_{i+1,1} & x_{i+1,0} \eta-x_{i+1,2}
\end{array}\right\| \ll X_{i+1} L_{t-1} L_{\imath} .
$$


Lemma 2.3.3 Let $\xi, \eta \in \mathbb{R}$ be such that $1, \xi, \eta$ are linearly independent over $\mathbb{Q}$. Let $\left(\mathbf{x}_{i}\right)_{1 \geq 1}$ be a sequence of minimal points associated to $(1, \xi, \eta)$. Denote by $\mathbf{I}$ the set of all indices $i \geq 2$ for which $\mathbf{x}_{i-1}, \mathbf{x}_{i}, \mathbf{x}_{i+1}$ are linearly independent. Then $\mathbf{I}$ is an infinite set.

Proof: $\quad$ For each $i \geq 2$, denote by $W$, the subspace of $\mathbb{R}^{3}$ of dimension 2 generated by $\mathbf{x}_{t-1}$ and $\mathbf{x}_{1}$. Suppose $\mathbf{I}$ is finite, then it either has a maximal element $i_{0}$, or it is empty in which case we take $i_{0}=1$. Then, for all $i>i_{0}$, the points $\mathbf{x}_{\imath-1}, \mathbf{x}_{i}, \mathbf{x}_{i+1}$ are linearly dependent. This is equivalent to saying that for all $i>i_{0}$ we have $W_{1}=W_{2+1}$, which implies that there is a plane $W$ such that $W=W_{2}$ for all $i>i_{0}$. Let $a x_{0}+b x_{1}+c x_{2}=0$ be an equation of $W$ with $a, b, c \in \mathbb{Z}$ not all zero. This gives

$$
a x_{i, 0}+b x_{i, 1}+c x_{\imath, 2}=0
$$

for all $i>i_{0}$ and therefore

$$
\left|x_{i, 0}(a+b \xi+c \eta)\right|=\left|b\left(x_{i, 0} \xi-x_{i, 1}\right)+c\left(x_{i, 0} \eta-x_{i, 2}\right)\right| \ll L\left(\mathbf{x}_{i}\right) .
$$

Since $a+b \xi+c \eta \neq 0$ by hypothesis and since $\left|x_{\imath, 0}\right| \asymp\left\|\mathbf{x}_{i}\right\|$ by Lemma 2.1 .5 we obtain

$$
\left\|\mathbf{x}_{i}\right\| \asymp L\left(\mathbf{x}_{i}\right) .
$$

However, the left hand side tends to infinity and the right hand side tends to zero as $i \rightarrow 0$. This is a contradiction.

Combining Lemmas 2.3 .2 and 2.3.1, we obtain:

Lemma 2.3.4 Let $\xi, \eta \in \mathbb{R}$ be such that $1, \xi, \eta$ are linearly independent over $\mathbb{Q}$. Let $\left(\mathbf{x}_{i}\right)_{2 \geq 1}$ be a sequence of minimal points associated to $(1, \xi, \eta)$. Denote by $\mathbf{I}$ the set of 
all indices $i \geq 2$ for which $\mathbf{x}_{\imath-1}, \mathbf{x}_{2}, \mathbf{x}_{\imath+1}$ are linearly independent. Suppose that $\lambda>0$ is an exponent of approximation to $(1, \xi, \eta)$. Then, for each $i \in \mathbf{I}$, we have

$$
1 \ll X_{i}^{-\lambda} X_{\imath+1}^{1-\lambda}
$$

Theorem 2.3.5 Let $\xi, \eta \in \mathbb{R}$ be such that $1, \xi, \eta$ are linearly independent over $\mathbb{Q}$. If $\lambda$ is an exponent, of approximation to $(1, \xi, \eta)$, then $\lambda<1$.

Proof: $\quad$ Suppose by contradiction that $\lambda \geq 1$. Let $\left(\mathbf{x}_{2}\right)_{i \geq 1}$ be a sequence of minimal points associated to $(1 . \xi, \eta)$. Denote by $\mathbf{I}$ the set of all indices $i \geq 2$ for which $\mathbf{x}_{t-1} \cdot \mathbf{x}_{\imath} \cdot \mathbf{x}_{i+1}$ are linearly independent. By Lemma 2.3.3. the set $\mathbf{I}$ has infinitely many elements. This is impossible in view of Lemma 2.3.4.

The next basic estimates will be useful throughout this thesis. They will allow us to simplify many computations.

Lemma 2.3.6 Let $\xi, \eta \in \mathbb{R}$ be such that $1, \xi, \eta$ are linearly independent, over $\mathbb{Q}$. Let $\left(\mathbf{x}_{1}\right)_{i \geq 1}$ be a sequence of minimal points associated to $(1, \xi . \eta)$. Denote by $\mathbf{I}$ the set of all indices $i \geq 2$ for which $\mathbf{x}_{i-1} \cdot \mathbf{x}_{i}, \mathbf{x}_{i+1}$ are linearly independent. Suppose that $\lambda>2 / 3$ is an exponent of approximation to $(1, \xi, \eta)$. Then for any $i \in \mathbf{I}$ we have

$$
X_{\imath}^{2} \ll X_{i+1}, \quad L_{\imath} \ll L_{i-1}^{2}, \quad L_{i} \ll X_{i}^{-4 / 3}
$$

Proof: We note that $2(1-\lambda) / \lambda<1$ since $\lambda>2 / 3$. Then, by Lemma 2.3 .4 we have

$$
X_{\imath}^{2} \ll X_{i+1}^{2(1-\lambda) / \lambda} \ll X_{i+1}
$$

We also find using Lemmas 2.3.1 and 2.3.2 that

$$
L_{\imath} \ll L_{i}\left(X_{i+1} L_{i} L_{i-1}\right)^{2} \ll L_{i}^{1-2(1-\lambda) / \lambda} L_{i-1}^{2} \leq L_{i-1}^{2}
$$


Finally, by Lemma 2.3.1

$$
L_{2} \ll X_{\imath+1}^{-\lambda} \ll X_{r}^{-2 \lambda}<X_{\imath}^{-4 / 3}
$$

In particular. if $\iota<J$ are consecutive elements of $\mathbf{I}$. the first two estimates of Lemma 2.3 .6 imply that $X_{\imath}^{2} \ll X_{\jmath}$ and $L_{\jmath} \ll L_{\imath}^{2}$. 


\section{Chapter 3}

\section{The function $\varphi$ and a first estimate}

To prove Theorem 1.1.1. Davenport and Schmidt proceed by contradiction. They suppose that, for any $c>0$. the inequalities of their theorem have a solution for cach $X$ sufficiently large. They then construct a sequence of minimal points $\left(x_{1}\right)_{\imath \geq 1}$ associated to $\left(1 . \xi . \xi^{2}\right)$ similar to our construction in Chapter 2. They first show that

$$
\operatorname{det}\left(\mathbf{x}_{\imath}\right)=\left|\begin{array}{ll}
x_{l, 0} & x_{r, 1} \\
x_{r, 1} & x_{\imath, 2}
\end{array}\right| \neq 0
$$

for all $\imath$ sufficiently large. Using a result similar to Lemma 2.3.1, they conclude that

$$
1 \leq\left|\operatorname{det}\left(\mathbf{x}_{2}\right)\right| \ll X_{\imath} L, \ll c X_{2} X_{\imath+1}^{-1 / \uparrow}
$$

where the lower bound comes from the fact that $\operatorname{det}\left(\mathbf{x}_{2}\right)$ is a non-zero integer. Then, they show as we do in Lemma 2.3.3 that, for infinitely many indices ?, the points $\mathbf{x}_{\imath-1}, \mathbf{x}_{\imath}, \mathbf{x}_{\imath+1}$ are linearly independent. So, their determinant is a non-zero integer and arguing as we did in Lemma 2.3.4 they find

$$
1 \ll\left|\operatorname{det}\left(\mathbf{x}_{1-1}, \mathbf{x}_{\imath} \cdot \mathbf{x}_{\imath+1}\right)\right| \ll X_{\imath+1} L_{2} L_{\imath-1} \ll c^{2} X_{\imath+1}^{1-1 / \gamma} X_{\imath}^{1 / \gamma}
$$

Combining these results they derive a contradiction if $c$ is sufficiently small.

Adapting the ideas of Davenport and Schmidt described above, we will show the following result: 
Theorem 3.7 Let $\xi \in \mathbb{R}$ be such that $1, \xi, \xi^{3}$ are linearly independent over $\mathbb{Q}$. Then $\lambda=\sqrt{3}-1$ is a measure of approximation to $\left(1, \xi, \xi^{3}\right)$.

We first introduce a polynomial which we will use throughont this paper, and which plays the role of the determinant in the work of Davenport and Schmidt:

Definition 3.8 We set

$$
\varphi(\mathbf{x}, \mathbf{y} . \mathbf{z}):=\left|\begin{array}{cc}
x_{0} y_{0} & x_{1} y_{1} \\
z_{1} & z_{2}
\end{array}\right| . \quad \varphi(\mathbf{x}):=\varphi(\mathbf{x}, \mathbf{x}, \mathbf{x})
$$

where $\mathbf{x}=\left(x_{0}, x_{1}, x_{2}\right), \mathbf{y}=\left(y_{0}, y_{1}, y_{2}\right), \mathbf{z}=\left(z_{0}, z_{1}, z_{2}\right)$.

Lemma 3.9 Let $\xi \in \mathbb{R}$ be such that $1, \xi, \xi^{3}$ are linearly independent over $\mathbb{Q}$. Let $\left(\mathbf{x}_{1}\right)_{i \geq 1}$ be a sequence of minimal points associated to $\left(1, \xi, \xi^{3}\right)$. Suppose that $\lambda>2 / 3$ is an exponent of approximation to $\left(1, \xi, \xi^{3}\right)$. Then. for each sufficiently large $i$. we have

$$
\varphi\left(\mathbf{x}_{i}\right)=\left|\begin{array}{cc}
x_{i, 0}^{2} & x_{i, 1}^{2} \\
x_{i, 1} & x_{i, 2}
\end{array}\right| \neq 0
$$

Proof: If the result were false we would have $x_{i, 1}^{3}=x_{i, 0}^{2} x_{i, 2}$. Since $x_{i .0} \neq 0$, this is equivalent to

$$
\frac{x_{i, 1}}{x_{i, 0}}=\frac{n}{m} \quad \text { and } \quad \frac{x_{i, 2}}{x_{i, 0}}=\left(\frac{x_{\imath .1}}{x_{i, 0}}\right)^{3}=\frac{n^{3}}{m^{3}}
$$

where $m, n$ are integers with $m \neq 0$ and $\operatorname{gcd}(m, n)=1$. It follows that

$$
\left(1, \frac{x_{i, 1}}{x_{i, 0}}, \frac{x_{\imath, 2}}{x_{i, 0}}\right)=\left(1 . \frac{n}{m}, \frac{n^{3}}{m^{3}}\right) \Rightarrow\left(x_{i, 0}, x_{i, 1}, x_{i, 2}\right) \propto\left(m^{3}, m^{2} n, n^{3}\right) .
$$

Since $x_{i, 0}, x_{i, 1}, x_{i, 2}$ have no common factors, they then take the form

$$
x_{i, 0}=\epsilon m^{3}, \quad x_{i .1}=\epsilon m^{2} n, \quad x_{\imath, 2}=\epsilon n^{3},
$$

where $\epsilon \in\{ \pm 1\}$. For $i$ large enough, $\left|x_{1, j}\right| \asymp X_{1}$ for $j=0,1,2$, and this gives

$$
|m| \asymp|n| \asymp X_{i}^{1 / 3} .
$$


Since $\left|\epsilon m^{2}(m \xi-n)\right|=\left|x_{r .0} \xi-x_{1,1}\right| \leq L_{r}$ we obtain

$$
|m \xi-n| \ll X_{\imath}^{-2 / 3} L_{\imath}
$$

and this implies

$$
\left|m^{2} \xi^{2}-n^{2}\right|=|m \xi-n||m \xi+n| \ll X_{\imath}^{-1 / 3} L_{\imath}
$$

The vectors $\mathrm{x}_{1-1}, \mathrm{x}_{2}$ are linearly independent so the matrix

$$
\left(\begin{array}{ccc}
x_{\imath-1,0} & x_{\imath-1,1} & x_{\imath-1,2} \\
x_{\imath 0} & x_{\imath, 1} & x_{\imath, 2}
\end{array}\right)
$$

has rank 2 and therefore has a non-zero $2 \times 2$ minor. For 7 large enough, $x_{t-11}$ and $x_{r, 1}$ are not zero, so we have

$$
\left|\begin{array}{cc}
x_{\imath-1,0} & x_{\imath-11} \\
x_{\imath, 0} & x_{\imath, 1}
\end{array}\right| \neq 0 \text { or } \quad\left|\begin{array}{cc}
x_{\imath-1,1} & x_{\imath-12} \\
x_{\imath, 1} & x_{22}
\end{array}\right| \neq 0
$$

Suppose that the first inequality of (3.3) holds. We then have

$$
\left|\begin{array}{cc}
x_{\imath-1,0} & x_{\imath-1.1} \\
m & n
\end{array}\right| \neq 0
$$

Using (3.1) and Lemma 2.3.1 we obtain

$$
\left\|\begin{array}{cc}
x_{\imath-1,0} & x_{\imath-1,0} \xi-x_{1-1,1} \\
m & m \xi-n
\end{array}\right\| \ll X_{\imath-1} X_{\imath}^{-2 / 3} L_{\imath}+X_{\imath}^{1 / 3} L_{\imath-1} \ll X_{\imath}^{1 / 3} L_{\imath-1} \ll X_{2}^{1 / 3-\lambda}
$$

Since $\lambda>1 / 3$, this contradicts (3.4) if $i$ is sufficiently large.

Suppose that the second inequality of (3.3) holds. We then have

$$
\left|\begin{array}{cc}
x_{\imath-1,1} & x_{\imath-1.2} \\
m^{2} & n^{2}
\end{array}\right| \neq 0
$$

Using (3.2) and Lemma 2.3.1 we obtain

$$
\left\|\begin{array}{cc}
x_{\imath-1.1} & x_{\imath-1,1} \xi^{2}-x_{\imath-1,2} \\
m^{2} & m^{2} \xi^{2}-n^{2}
\end{array}\right\| \ll X_{\imath-1} X_{\imath}^{-1 / 3} L_{\imath}+X_{\imath}^{2 / 3} L_{\imath-1} \ll X_{\imath}^{2 / 3} L_{\imath-1} \ll X_{\imath}^{2 / 3-\lambda} .
$$


Since $\lambda>2 / 3$, this contradicts (3.5) if $i$ is sufficiently large.

Lemma 3.10 Let $\xi \in \mathbb{R}$ be such that $1, \xi, \xi^{3}$ are linearly indcpendent over $\mathbb{Q}$. Let $\left(\mathbf{x}_{2}\right)_{1 \geq 1}$ be a sequence of minimal points associated to $\left(1, \xi, \xi^{3}\right)$. Suppose that $\lambda>2 / 3$ is an exponent of approximation to $\left(1, \xi, \xi^{3}\right)$. Then, for each $i$, we have

$$
1 \ll X_{i}^{2} L_{i}
$$

Proof: We find

$$
\begin{aligned}
\left|\varphi\left(\mathbf{x}_{\imath}\right)\right| & =\left\|\begin{array}{ll}
x_{i, 0}^{2} & x_{\imath .1}^{2} \\
x_{\imath, 1} & x_{i, 2}
\end{array}\right\|=\left\|\begin{array}{ll}
x_{i, 0}^{2} & x_{i, 0}^{2} \xi^{2}-x_{i, 1}^{2} \\
x_{\imath, 1} & x_{\imath, 1} \xi^{2}-x_{i, 2}
\end{array}\right\| \\
& \ll X_{i}^{2} L_{i}+X_{\imath}\left|x_{i, 0} \xi-x_{\imath, 1} \| x_{r, 0} \xi+x_{i, 1}\right| \ll X_{\imath}^{2} L_{i} .
\end{aligned}
$$

But by Lemma $3.9, \varphi\left(\mathbf{x}_{i}\right)$ is a non-zero integer. So its absolute value is $\geq 1$.

Proof of Theorem 3.7: $\quad$ Let $\left(\mathbf{x}_{i}\right)_{i \geq 1}$ be a sequence of minimal points associated to $(1, \xi . \eta)$. Denote by $\mathbf{I}$ the infinite set of all indices $i \geq 2$ for which $\mathbf{x}_{i-1}, \mathbf{x}_{1}, \mathbf{x}_{2+1}$ are linearly independent. Suppose on the contrary that $\lambda$ is not a measure of approximation. Then for every $c>0, \lambda$ is an exponent of approximation.

Let $i \in \mathbf{I}$ with $i$ arbitrarily large. We then find:

$$
\begin{aligned}
1 & \ll\left(X_{i}^{2} L_{i}\right)^{\lambda} \\
& \ll\left(X_{i}^{2} L_{i}\right)^{\lambda}\left(X_{\imath+1} L_{\imath-1} L_{\imath}\right)^{2} \\
& \ll c^{4+\lambda} X_{\imath+1}^{2-2 \lambda-\lambda^{2}}
\end{aligned}
$$

Since $\lambda=\sqrt{3}-1$ is a root of $x^{2}+2 x-2$, we have $X_{i+1}^{2-2 \lambda-\lambda^{2}}=1$, and so the latter estimate becomes $1 \ll c^{4-\lambda}$. This is a contradiction. 


\section{Chapter 4}

\section{A family of planes in $\mathbb{R}^{3}$}

Define the height of a $2 \times 3$ matrix $A$, denoted $\|A\|$, to be the maximal absolute value of its minors of order 2, and define the height of a 2-dimensional subspace $V$ of $\mathbb{Q}^{3}$, denoted $H(V)$, to be the height of any $2 \times 3$ matrix whose rows form a basis of $V \cap \mathbb{Z}^{3}$.

The next result generalizes Lemma 4.1 of [7]. The first part is essentially due to Davenport and Schmidt (see the proof of Lemma 2 of [3]).

Lemma 4.1 Let $\xi, \eta \in \mathbb{R}$ be such that $1, \xi, \eta$ are linearly independent over $\mathbb{Q}$. Let $\left(\mathbf{x}_{i}\right)_{l \geq 1}$ be a sequence of minimal points associated to $(1, \xi, \eta)$. As in the proof of Lemma 2.3.3, we denote by $W_{\imath}$ the subspace of $\mathbb{R}^{3}$ of dimension 2 generated by $\mathbf{x}_{i-1}$ and $\mathbf{x}_{i}$. For each $i \geq 2$, we have that $\left\{\mathbf{x}_{\imath-1}, \mathbf{x}_{i}\right\}$ is a basis of $W_{i} \cap \mathbb{Z}^{3}$ over $\mathbb{Z}$ and $H\left(W_{i}\right) \asymp\left\|\mathbf{x}_{i}\right\| L\left(\mathbf{x}_{i-1}\right)$.

Proof: Suppose on the contrary that $\left\{\mathbf{x}_{\imath-1} \cdot \mathbf{x}_{i}\right\}$ is not a basis of $W_{1} \cap \mathbb{Z}^{3}$. Then there exists a non-zero point $\mathbf{x}=\left(x_{0}, x_{1}, x_{2}\right) \in W_{i} \cap \mathbb{Z}^{3}$ of the form $\mathbf{x}=r \mathbf{x}_{r-1}+s \mathbf{x}_{r}$ for rational numbers $r$ and $s$ with $\max \{|r|,|s|\} \leq 1 / 2$. We get

$$
\|\mathbf{x}\| \leq|r|\left\|\mathbf{x}_{i-1}\right\|+|s|\left\|\mathbf{x}_{i}\right\|<\left\|\mathbf{x}_{i}\right\| \quad \text { and } \quad L(\mathbf{x}) \leq|r| L\left(\mathbf{x}_{i-1}\right)+|s| L\left(\mathbf{x}_{i}\right)<L\left(\mathbf{x}_{i-1}\right)
$$

which contradicts the fact that $\mathbf{x}_{i-1}$ and $\mathbf{x}_{i}$ are consecutive minimal points. Thus. $\left\{\mathbf{x}_{1-1}, \mathbf{x}_{2}\right\}$ is a basis of $W_{i} \cap \mathbb{Z}^{3}$. 
We note that

$$
\begin{aligned}
& \left\|\begin{array}{cc}
x_{\imath-1,0} & x_{\imath-11} \\
x_{\imath, 0} & x_{\imath, 1}
\end{array}\right\|=\left\|\begin{array}{cc}
x_{\imath-1,0} & x_{\imath-1,0} \xi-x_{\imath-1,1} \\
x_{\imath, 0} & x_{\imath, 0} \xi-x_{\imath, 1}
\end{array}\right\| \ll\left\|\mathbf{x}_{\imath-1}\right\| L\left(\mathbf{x}_{\imath}\right)+\left\|\mathbf{x}_{\imath}\right\| L\left(\mathbf{x}_{\imath-1}\right), \\
& \left\|\begin{array}{cc}
x_{\imath-1,0} & x_{\imath-12} \\
x_{\imath, 0} & x_{\imath, 2}
\end{array}\right\|=\left\|\begin{array}{cc}
x_{\imath-1,0} & x_{\imath-1,0} \eta-x_{\imath-1,2} \\
x_{\imath, 0} & x_{\imath, 0} \eta-x_{\imath, 2}
\end{array}\right\| \ll \mathbf{x}_{\imath-1}\left\|L\left(\mathbf{x}_{\imath}\right)+\right\| \mathbf{x}_{\imath} \| L\left(\mathbf{x}_{\imath-1}\right), \\
& \left\|\begin{array}{cc}
x_{\imath-1,1} & x_{\imath-12} \\
x_{\imath, 1} & x_{\imath, 2}
\end{array}\right\|=\left\|\begin{array}{cc}
x_{\imath-1,1} & x_{\imath-1,1} \eta / \xi-x_{\imath-1,2} \\
x_{\imath, 1} & x_{\imath, 1} \eta / \xi-x_{\imath, 2}
\end{array}\right\| \ll\left\|\mathbf{x}_{\imath-1}\right\| L\left(\mathbf{x}_{\imath}\right)+\left\|\mathbf{x}_{\imath}\right\| L\left(\mathbf{x}_{\imath-1}\right) .
\end{aligned}
$$

Therefore,

$$
H\left(W_{\imath}\right)=\left\|\left(\begin{array}{ccc}
x_{\imath-1.0} & x_{2-1,1} & x_{\imath-1,2} \\
x_{\imath 0} & x_{\imath, 1} & x_{\imath, 2}
\end{array}\right)\right\| \ll\left\|\mathbf{x}_{\imath-1}\right\| L\left(\mathbf{x}_{\imath}\right)+\left\|\mathbf{x}_{\imath}\right\| L\left(\mathbf{x}_{\imath-1}\right) \ll\left\|\mathbf{x}_{\imath}\right\| L\left(\mathbf{x}_{\imath-1}\right) .
$$

Write $u_{1}=x_{\imath-11}-x_{\imath-1.0} \xi, u_{2}=x_{\imath-1.2}-x_{1-1,0} \eta$ and $v_{1}=x_{\imath, 1}-x_{\imath, 0} \xi . v_{2}=x_{\imath, 2}-x_{\imath, 0} \eta$.

Choose an index $J$ for which $\left|u_{j}\right|=L\left(\mathbf{x}_{\imath-1}\right)$. This gives

$$
\begin{aligned}
H\left(W_{\imath}\right) & \geq\left\|\begin{array}{cc}
x_{\imath-1.0} & x_{\imath-1, \jmath} \\
x_{\imath, 0} & x_{\imath, 3}
\end{array}\right\|=\left|x_{\imath-1,0} v_{\jmath}-x_{\imath, 0} u_{\jmath}\right| \\
& \geq\left|x_{\imath, 0}\right|\left|u_{\imath}\right|-\left|x_{\imath-1,0}\right|\left|r_{\jmath}\right| \geq\left(\left|x_{\imath, 0}\right|-\left|x_{\imath-1,0}\right|\right) L\left(\mathbf{x}_{\imath-1}\right) .
\end{aligned}
$$

As we observed in Chapter 2, for 1 sufficiently large and for an appropriate choice of $\epsilon \in\{ \pm 1\}$. the points $\mathbf{x}_{\imath-1}$ and $\epsilon \mathbf{x}_{1}$ lie in the same octant. Then the point $\mathbf{z}=\mathbf{x}_{i}-t \mathbf{x}_{t-1}$ satisfies $1 \leq\|\mathbf{z}\|<\left\|\mathbf{x}_{\imath}\right\|$. However, since $\mathbf{x}_{\imath-1}$ and $\mathbf{x}_{\imath}$ are consecutive minimal points, $\mathbf{x}_{\imath-1}$ is a minimal point corresponding to $\max \left\{\left\|\mathbf{x}_{\imath-1}\right\|,\|\mathbf{z}\|\right\}$. This means that $L(\mathbf{z}) \geq$ $L\left(\mathbf{x}_{1-1}\right)$. Choose an index $k$ for which $\left|v_{k}-\epsilon u_{k}\right|=L(\mathbf{z}) \geq L\left(\mathbf{x}_{1-1}\right)$. Then. we also find that

$$
\begin{aligned}
H\left(W_{\imath}\right) & \geq\left|x_{\imath-1,0} v_{k}-x_{\imath, 0} u_{k}\right|=\left|x_{\imath-1,0}\left(v_{k}-\epsilon u_{k}\right)-\left(x_{\imath, 0}-\epsilon x_{\imath-1,0}\right) u_{k}\right| \\
& \geq\left|x_{\imath-1,0}\right| L\left(\mathbf{x}_{\imath-1}\right)-\left|x_{\imath, 0}-\epsilon x_{\imath-1.0}\right| L\left(\mathbf{x}_{\imath-1}\right) .
\end{aligned}
$$

Since from Lemma 2.2 .4 we have $\left|x_{\imath, 0}\right| \geq\left|x_{2-1,0}\right|$, it follows that $\left|x_{\imath, 0}-+x_{1-1,0}\right|=$ $\left|x_{10}\right|-\left|x_{1-1,0}\right|$ and we get

$$
H\left(W_{1}\right) \geq\left|x_{i-1,0}\right| L\left(\mathbf{x}_{2-1}\right)-\left(\left|x_{1,0}\right|-\left|x_{\imath-1,0}\right|\right) L\left(\mathbf{x}_{i-1}\right) .
$$


Combining the inequalities (4.1) and (4.2) we get

$$
3 H\left(W_{i}\right) \geq\left|x_{i-1,0}\right| L\left(\mathbf{x}_{i-1}\right)+\left(\left|x_{i, 0}\right|-\left|x_{i-1,0}\right|\right) L\left(\mathbf{x}_{i-1}\right)=\left|x_{i, 0}\right| L\left(\mathbf{x}_{i-1}\right) .
$$

Therefore we have

$$
\left\|\mathbf{x}_{i}\right\| L\left(\mathbf{x}_{i-1}\right) \gg 3 H\left(W_{i}\right) \geq\left|x_{i, 0}\right| L\left(\mathbf{x}_{i-1}\right) \asymp\left\|\mathbf{x}_{i}\right\| L\left(\mathbf{x}_{i-1}\right)
$$

which means that $H\left(W_{i}\right) \asymp\left\|\mathbf{x}_{i}\right\| L\left(\mathbf{x}_{i-1}\right)$.

From this lemma, we derive the following useful general estimates.

Corollary 4.2 Let $\xi . \eta \in \mathbb{R}$ be such that $1, \xi, \eta$ are linearly independent over $\mathbb{Q}$. Let $\left(\mathbf{x}_{i}\right)_{i \geq 1}$ be a sequence of minimal points associated to $(1, \xi, \eta)$. Denote by $\mathbf{I}$ the set of all indices $i \geq 2$ for which $\mathbf{x}_{i-1}, \mathbf{x}_{i}, \mathbf{x}_{i+1}$ are linearly independent. Then for any pair $i<j$ consecutive elements of $\mathbf{I}$ we have

$$
X_{i+1} L_{i} \asymp X_{j} L_{j-1}
$$

Proof: $\quad$ Since $i<j$ are consecutive elements of $\mathbf{I}$, we have that $W_{i+1}=W_{j}$ and from the lemma we obtain

$$
X_{i+1} L_{i} \asymp H\left(W_{i+1}\right)=H\left(W_{j}\right) \asymp X_{j} L_{j-1}
$$

Corollary 4.3 Let $\xi, \eta \in \mathbb{R}$ be such that $1, \xi, \eta$ are linearly independent over $\mathbb{Q}$. Let $\left(\mathbf{x}_{i}\right)_{i \geq 1}$ be a sequence of minimal points associated to $(1, \xi, \eta)$. Denote by $\mathbf{I}$ the set of all indices $i \geq 2$ for which $\mathbf{x}_{i-1}, \mathbf{x}_{i}, \mathbf{x}_{i+1}$ are linearly independent. Suppose that $\lambda>0$ is an exponent of approximation to $(1, \xi, \eta)$. Then for any pair of consecutive elements $i<j$ of $\mathbf{I}$ we have

$$
X_{j} \ll\left(X_{i+1} X_{j+1}\right)^{1-\lambda} \quad \text { and } \quad L_{i} L_{j} \ll X_{j}^{-\lambda /(1-\lambda)} .
$$


Proof: We first note that:

$$
\begin{aligned}
1 & \ll X_{\jmath+1} L_{\jmath-1} L_{\jmath} & & \text { (Lemma 2.3.2) } \\
& \asymp X_{\jmath+1}\left(X_{\imath+1} L_{\imath} X_{\jmath}^{-1}\right) L_{\jmath} & & \text { (Corollary 4.2) } \\
& \ll X_{\imath+1}^{1-\lambda} X_{\jmath}^{-1} X_{\jmath+1}^{1-\lambda} & & \text { (Lemma 2.3.1) }
\end{aligned}
$$

This proves the first estimate of the corollary. The second follows from Lemma 2.3.1 and the first estimate:

$$
L_{\imath} L_{\jmath} \ll\left(X_{\imath+1} X_{\jmath+1}\right)^{-\lambda} \ll X_{\jmath}^{-\lambda /(1-\lambda)}
$$




\section{Chapter 5}

\section{The function $\Phi$ and the point $\psi$}

We now introduce further tools which will be used throughout this thesis. In this chapter. we use them to improve on Theorem 3.7 by showing that, if $\xi \in \mathbb{R}$ is such that $1 . \xi, \xi^{3}$ are linearly independent over $\mathbb{Q}$. then any exponent of approximation to $\left(1, \xi, \xi^{3}\right)$ is at most equal to the root of $7 x^{3}-2 x^{2}-5 x+2$ near 0.7277416 .

Definition 5.1 We set

$$
\begin{aligned}
\Phi(\mathbf{x}, \mathbf{y}, \mathbf{z}) & =\varphi(\mathbf{x}, \mathbf{y}, \mathbf{z})+\varphi(\mathbf{y}, \mathbf{z}, \mathbf{x})+\varphi(\mathbf{z}, \mathbf{x}, \mathbf{y}) \\
\psi(\mathbf{x}, \mathbf{y}) & =\Phi(\mathbf{x}, \mathbf{x}, \mathbf{y}) \mathbf{x}-2 \varphi(\mathbf{x}) \mathbf{y}
\end{aligned}
$$

The function $\psi$ constructs new points of $\mathbb{R}^{3}$ out of each pair of points of $\mathbb{R}^{3}$. The following proposition provides upper bounds for the norm and the proximity function $L(\cdot)$ of these points.

Proposition 5.2 For any $\mathrm{x}, \mathrm{y} \in \mathbb{R}^{3}$ we have:

$$
\begin{aligned}
\|\psi(\mathbf{x} \cdot \mathbf{y})\| & \ll\|\mathbf{x}\|^{3} L(\mathbf{y})+\|\mathbf{x}\|\|\mathbf{y}\| L(\mathbf{x})^{2} \\
L(\psi(\mathbf{x} \cdot \mathbf{y})) & \ll\|\mathbf{x}\|^{2} L(\mathbf{x}) L(\mathbf{y})+\|\mathbf{x}\|\|\mathbf{y}\| L(\mathbf{x})^{2}
\end{aligned}
$$


Proof: Let $\mathbf{x}=\left(x_{0}, x_{1}, x_{2}\right)$ and $\mathbf{y}=\left(y_{0}, y_{1}, y_{2}\right)$ be points of $\mathbb{R}^{3}$. Write $\underline{\xi}=\left(1, \xi . \xi^{3}\right)$ and define.

$$
\Delta \mathbf{x}=\mathbf{x}-x_{0} \underline{\underline{\xi}}=\left(0 . \Delta x_{1}, \Delta x_{2}\right) \quad \text { and } \quad \delta(\mathbf{x}):=\Phi(\mathbf{x}, \underline{\underline{\xi}}, \underline{\underline{\xi}})=\Delta x_{2}-3 \xi^{2} \Delta x_{1}
$$

We find the following developments:

$$
\begin{aligned}
\varphi(\mathbf{x}) & =x_{0}^{2} \delta(\mathbf{x})-3 \xi \Delta x_{1}^{2} x_{0}-\Delta x_{1}^{3} \\
\Phi(\mathbf{x}, \mathbf{x} . \mathbf{y}) & =x_{0}^{2} \delta(\mathbf{y})+2 x_{0} y_{0} \delta(\mathbf{x})-3 \xi y_{0} \Delta x_{1}^{2}-6 \xi x_{0} \Delta x_{1} \Delta y_{1}-3 \Delta x_{1}^{2} \Delta y_{1}
\end{aligned}
$$

Using these we get the following estimate for $\psi$ :

$$
\begin{aligned}
\psi(\mathbf{x} \cdot \mathbf{y})= & \Phi(\mathbf{x} \cdot \mathbf{x}, \mathbf{y})\left(x_{0} \underline{\underline{\xi}}+\Delta \mathbf{x}\right)-2 \varphi(\mathbf{x})\left(y_{0} \underline{\underline{\xi}}+\Delta \mathbf{y}\right) \\
= & \left(x_{0}^{2} \delta(\mathbf{y})+2 x_{0} y_{0} \delta(\mathbf{x})-3 \xi y_{0} \Delta x_{1}^{2}-6 \xi x_{0} \Delta x_{1} \Delta y_{1}-3 \Delta x_{1}^{2} \Delta y_{1}\right)\left(x_{0} \underline{\xi}+\Delta \mathbf{x}\right) \\
& -2\left(x_{0}^{2} \delta(\mathbf{x})-3 \xi \Delta x_{1}^{2} x_{0}-\Delta x_{1}^{3}\right)\left(y_{0} \underline{\underline{\xi}}+\Delta \mathbf{y}\right) \\
= & \left(x_{0}^{3} \delta(\mathbf{y})-6 \xi x_{0}^{2} \Delta x_{1} \Delta y_{1}+3 \xi x_{0} y_{0} \Delta x_{1}^{2}-3 x_{0} \Delta x_{1}^{2} \Delta y_{1}+2 y_{0} \Delta x_{1}^{3}\right) \underline{\underline{\xi}} \\
& +\mathcal{O}\left(\|\mathbf{x}\|^{2} L(\mathbf{x}) L(\mathbf{y})+\|\mathbf{x}\|\|\mathbf{y}\| L(\mathbf{x})^{2}\right)
\end{aligned}
$$

The conclusion follows upon noting that $L(\underline{\underline{\xi}})=0$.

In our context, these estimates lead to:

Lemma 5.3 Let $\xi \in \mathbb{R}$ be such that $1, \xi, \xi^{3}$ are linearly independent over $\mathbb{Q}$. Let $\left(\mathbf{x}_{i}\right)_{i \geq 1}$ be a sequence of minimal points associated to $\left(1, \xi, \xi^{3}\right)$. Denote by $\mathbf{I}$ the set of all indices $i \geq 2$ for which $\mathbf{x}_{i-1}, \mathbf{x}_{i}, \mathbf{x}_{i+1}$ are linearly independent. Suppose that $\lambda>2 / 3$ is an exponent of approximation to $\left(1, \xi, \xi^{3}\right)$. Then for any pair of consecutive elements $i<j$ in $\mathbf{I}$ we have

$$
\left\|\psi\left(\mathbf{x}_{i}, \mathbf{x}_{j}\right)\right\| \ll X_{i} X_{j} L_{i}^{2}
$$


Proof: By Proposition 5.2, we have $\left\|\psi\left(\mathbf{x}_{i}, \mathbf{x}_{j}\right)\right\| \ll X_{i}^{3} L_{j}+X_{i} X_{j} L_{i}^{2}$. So it suffices to show that $X_{i}^{3} L_{j} \ll X_{1} X_{j} L_{i}^{2}$. Since $i$ and $j$ are in $\mathbf{I}$, Lemma 2.3.6 gives indeed

$$
X_{i}^{3} L_{j} \ll X_{i} X_{i+1} L_{J} \ll X_{i} X_{i+1} L_{j-1}^{2} \leq X_{\imath} X_{j} L_{i}^{2}
$$

We now observe that the function $\psi$ has the following non-vanishing property.

Lemma 5.4 Lct $\xi \in \mathbb{R}$ be such that $1, \xi, \xi^{3}$ are linearly indcpendent over $\mathbb{Q}$. Let $\left(\mathbf{x}_{1}\right)_{i \geq 1}$ be a sequence of minimal points associated to $\left(1 . \xi, \xi^{3}\right)$. Suppose that $\lambda>2 / 3$ is an exponent of approximation to $\left(1, \xi, \xi^{3}\right)$. Then for any $i, j \geq 1$ wnth i sufficiently large we have.

$$
\psi\left(\mathbf{x}_{i}, \mathbf{x}_{j}\right) \neq 0
$$

Proof: By Lemma 3.9. we have $\varphi\left(\mathbf{x}_{\imath}\right) \neq 0$ for each $i$ sufficiently large. If $i=j$ we find

$$
\psi\left(\mathbf{x}_{i}, \mathbf{x}_{i}\right)=\varphi\left(\mathbf{x}_{i}\right) \mathbf{x}_{\imath} \neq 0
$$

Otherwise $i \neq j$, so $\mathbf{x}_{i}, \mathbf{x}_{j}$ are linearly independent and we obtain

$$
\psi\left(\mathbf{x}_{i}, \mathbf{x}_{j}\right)=\Phi\left(\mathbf{x}_{i}, \mathbf{x}_{i}, \mathbf{x}_{j}\right) \mathbf{x}_{i}-2 \varphi\left(\mathbf{x}_{i}\right) \mathbf{x}_{j} \neq 0
$$

Combining Lemmas 5.3 and 5.4 together with the fact that any non-zero integer point has norm at least 1 , we obtain immediately:

Corollary 5.5 Let $\xi \in \mathbb{R}$ be such that $1, \xi . \xi^{3}$ are linearly independent over $\mathbb{Q}$. Let $\left(\mathbf{x}_{i}\right)_{i \geq 1}$ be a sequence of minimal points associated to $\left(1, \xi, \xi^{3}\right)$. Denote by $\mathbf{I}$ the set of all indices $i \geq 2$ for which $\mathbf{x}_{i-1}, \mathbf{x}_{\imath}, \mathbf{x}_{\imath+1}$ are linearly independent. Suppose that 
$\lambda>2 / 3$ is an exponent of approxmation to $\left(1, \xi, \xi^{3}\right)$. Then for any par of conseculzve elements $i<j$ in I we have

$$
1 \ll X_{,} X_{3} L_{\imath}^{2}
$$

We are now ready to prove the main result of this chapter:

Theorem 5.6 Let $\xi \in \mathbb{R}$ be such that $1, \xi, \xi^{3}$ are linearly independent over $\mathbb{Q}$. If $\lambda$ is an exponent of upproximation to $\left(1, \xi . \xi^{3}\right)$, then $\lambda \leq \lambda_{0}$ where $\lambda_{0}$ is the largest real root of $7 x^{3}-2 x^{2}-5 x+2$, about 0.7277416 .

Proof: Suppose by contradiction that $\lambda>\lambda_{0}$. Let $\left(\mathbf{x}_{2}\right)_{2} \geq 1$ be a sequence of minimal points associated to $\left(1, \xi . \xi^{3}\right)$. Denote by $\mathbf{I}$ the set of all indices $? \geq 2$ for which $\mathbf{x}_{l-1}, \mathbf{x}_{\imath}, \mathbf{x}_{t+1}$ are linearly independent. Let $i<j$ be a pair of consecutive elements of $\mathbf{I}$ with $i$ arbitrarily large.

We first note that

$$
\begin{aligned}
X_{\jmath}^{\lambda} & \ll\left(X_{1+1} X_{\jmath+1}\right)^{\lambda(1-\lambda)} \\
& \ll X_{\imath+1}^{\lambda(1-\lambda)} X_{\jmath}^{2(1-\lambda)}
\end{aligned}
$$

which gives us that

$$
X_{1}^{3 \lambda-2} \ll X_{1-1}^{\lambda(1-\lambda)}
$$

Combining this result with Corollary 5.5 we obtain

$$
\begin{aligned}
1 & \ll\left(X_{\imath} X_{\jmath} L_{\imath}^{2}\right)^{\lambda(3 \lambda-2)} \\
& \ll\left(X_{\imath+1}^{1-\lambda} X_{\jmath}^{\lambda} L_{\imath}^{2 \lambda}\right)^{3 \lambda-2} \\
& \ll\left(X_{\imath+1}^{1-\lambda-2 \lambda^{2}} X_{\jmath}^{\lambda}\right)^{3 \lambda-2} \\
& \ll X_{\imath+1}^{\left(1-\lambda-2 \lambda^{2}\right)(3 \lambda-2)+\lambda^{2}(1-\lambda)} \\
& =X_{\imath+1}^{-\left(7 \lambda^{3}-2 \lambda^{2}-5 \lambda+2\right)} .
\end{aligned}
$$

Iowever, since $\lambda>\lambda_{0}$ the exponent of $X_{2+1}$ in the latter expression is negative and so that power of $X_{i+1}$ tends to 0 as $i \rightarrow \infty$. This is a contradiction. 
Since $\lambda_{0}<\sqrt{3}-1 \approx 0.732$, the theorem has the following immediate consequence.

Corollary 5.7 Let $\xi \in \mathbb{R}$ be such that $1, \xi, \xi^{3}$ are linearly independent over $\mathbb{Q}$. Then $\lambda=\sqrt{3}-1$ is not an exponent of approximation to $\left(1 . \xi, \xi^{3}\right)$. 


\section{Chapter 6}

\section{Intermediate points and large systems of inequalities}

In this chapter we show that, if $\xi \in \mathbb{R}$ is such that $1, \xi, \xi^{3}$ are linearly independent over $\mathbb{Q}$, then any an exponent of approximation to $\left(1, \xi, \xi^{3}\right)$ is at most $8 / 11$. This improves on Theorem 5.6 since $8 / 11=0 . \overline{72}<0.7277$.

We begin by looking at a special case:

Proposition 6.1 Let $\xi \in \mathbb{R}$ be such that $1, \xi, \xi^{3}$ are linearly independcnt over $\mathbb{Q}$. Let $\left(\mathbf{x}_{i}\right)_{1 \geq 1}$ be a sequence of minimal points associaled to $\left(1, \xi, \xi^{3}\right)$. Denote by $\mathbf{I}$ the set of all indices $i \geq 2$ for which $\mathbf{x}_{i-1}, \mathbf{x}_{i}, \mathbf{x}_{i+1}$ are linearly independent. Suppose that for infinitely many $i \in \mathbf{I}$, we have $i+1 \in \mathbf{I}$. Then $\lambda=\sqrt{2} / 2$ is a measure of approximation to $\left(1 . \xi \cdot \xi^{3}\right)$.

Proof: Suppose on the contrary that $\lambda$ is not a measure of approximation to $\left(1, \xi, \xi^{3}\right)$. Then for every $c>0, \lambda$ is an exponent of approximation to $\left(1, \xi, \xi^{3}\right)$.

Let $i \in \mathbf{I}$ be such that $i+1 \in \mathbf{I}$. Lemma 2.3 .4 shows that $1 \ll X_{i}^{-\lambda} X_{i+1}^{1-\lambda}$ and 
Corollary 5.5 gives $1 \ll X_{i} X_{i+1} L_{i}^{2}$. For such an $i$ we then have

$$
\begin{aligned}
1 & \ll\left(X_{i} X_{\imath+1} L_{i}^{2}\right)^{(1-\lambda)} \ll c^{2(1-\lambda)} X_{i}^{(1-\lambda)} X_{\imath+1}^{(1-2 \lambda)(1-\lambda)} \\
& \ll c^{2(1-\lambda)} X_{i}^{(1-\lambda)-\lambda(2 \lambda-1)}=c^{2(1-\lambda)} X_{i}^{1-2 \lambda^{2}}
\end{aligned}
$$

Since $\lambda=\sqrt{2} / 2$ is a root of $2 x^{2}-1$ we have $X_{2}^{1-2 \lambda^{2}}=1$ and so for a choice of $c>0$ sufficiently small this is a contradiction.

From this result it is clear that, for any $\xi \in \mathbb{R}$ such that $1, \xi, \xi^{3}$ are linearly inclependent over $\mathbb{Q}$, if $\lambda>\sqrt{2} / 2$ is an exponent of approximation to $\left(1, \xi, \xi^{3}\right)$ then for each $i \in \mathbf{I}$ sufficiently large we have $i+1 \notin \mathbf{I}$.

At this point, we pause and make the following general observation about systems of linear inequalities:

Proposition 6.2 Let $m, n \in \mathbb{Z}_{\geq 0}$ and $a_{l, l}, b_{\imath} \in \mathbb{R}$ for $i=0, \ldots, m$ and $j=0 \ldots n$. Supposc the system of linear incqualities

$$
\sum_{j=0}^{n} a_{\imath, j} x_{j}+b_{i} \leq \varepsilon_{\imath}, \quad i=0, \ldots, m
$$

has a solution $\left(x_{0}, \ldots, x_{n}\right) \in \mathbb{R}^{n+1}$ for any choice of $\varepsilon_{0} \ldots, \varepsilon_{m}>0$. Then it has a solution for $\varepsilon_{0}=\cdots=\varepsilon_{m}=0$.

Proof: We note that for $0 \leq k \leq m$, the system of inequalities

$$
\left\{\begin{aligned}
\sum_{j=0}^{n}-a_{i, j} x_{\jmath}-b_{\imath}+\varepsilon_{i} \geq 0, & i=0, \ldots, k-1 \\
\sum_{j=0}^{n}-a_{i, j} x_{j}-b_{i} \geq 0, & i=k+1, \ldots, m
\end{aligned}\right.
$$

together with the linear functional

$$
\theta=\sum_{\jmath=0}^{n} a_{k, \jmath} x_{\jmath}
$$


is a linear program. According to Theorem 1 of $\S 8.1$ of [6], either the set of admissible solutions (i.e. solutions to the syst em of inequalities) is empty, or the set of admissible solutions has at least one minimal solution (i.e. a solution for which $\theta$ takes its minimum value), or there are admissible solutions for which $\theta$ is arbitrarily small (i.e. can be made smaller than any given real number, positive or negative).

We now show by reverse induction on $k$ that the system

$$
\left\{\begin{array}{l}
\sum_{j=0}^{n} a_{i, j} x_{\jmath}+b_{i} \leq \varepsilon_{i}, \quad i=0, \ldots, k \\
\sum_{j=0}^{n} a_{i . j} x_{\jmath}+b_{i} \leq 0 . \quad i=k+1, \ldots, m
\end{array}\right.
$$

has a solution for every choice of $\varepsilon_{0}, \ldots, \varepsilon_{L}>0$.

For $k=m$, this is true by hypothesis. Suppose now that $0 \leq k \leq m$ and that the system $(6.2)$ has a solution for any choice of $\varepsilon_{0} \ldots, \varepsilon_{k}>0$. Fix $\varepsilon_{0} \ldots, \varepsilon_{k-1}>0$. Then. the set of admissible solutions to (6.1) is non-empty. If there are admissible solntions for which $\theta$ is arbitrarily small then there is a solution for which $\theta+b_{k} \leq 0$. We are left with the case where the set of admissible solutions has at least one minimal solution. Let $\theta_{0}$ be the value of $\theta$ for a minimal solution. Since we have solutions such that $\theta+b_{k} \leq \epsilon_{k}$ for every $\epsilon_{k}>0$, then we must have $\theta_{0}+b_{k} \leq 0$ and so a minimal solution satisfies $\theta+b_{k} \leq 0$.

Using this lemma we can deduce the following proposition.

Proposition 6.3 Fix $n \in \mathbb{Z}_{\geq 0}$. Let $\xi \in \mathbb{R}$ be such that $1, \xi, \xi^{3}$ are linearly independent over $\mathbb{Q}$. Suppose that $\lambda>2 / 3$ is an exponent of approximation to $\left(1, \xi, \xi^{3}\right)$. 
Then the followng system of $4 n+4$ inequalitıes.

$$
\begin{gathered}
B_{0}+\lambda A_{0} \leq 0 \\
0 \leq 2 A_{2 n}+B_{2 n+1} \\
0 \leq A_{2 n+1}+B_{2 n}+B_{2 n+1} \\
B_{2 n+1}+\lambda A_{2 n+1} \leq 0 \\
k=0, \ldots, n-1\left\{\begin{array}{l}
A_{2 k+1}+B_{2 k+1}=A_{2 k+2}+B_{2 k+2} \\
0 \leq A_{2 k}+A_{2 k+2}+2 B_{2 k+1} \\
0 \leq A_{2 k+1}+B_{2 k}+B_{2 k+1} \\
B_{2 k+1}+\lambda A_{2 k+1} \leq 0
\end{array}\right.
\end{gathered}
$$

has a solution $\left(A_{0} \ldots A_{2 n+1}, B_{0}, \ldots, B_{2 n+1}\right)$ in $\mathbb{R}^{4 n+4}$ with $A_{0}=1$.

Proof: Let $\left(x_{1}\right)_{2 \geq 1}$ be a sequence of minimal points associated to $\left(1, \xi, \xi^{3}\right)$. Denote by $\mathbf{I}$ the set of all indices $\imath \geq 2$ for which $\mathbf{x}_{\imath-1}, \mathbf{x}_{\imath}, \mathbf{x}_{\imath+1}$ are linearly independent. Let $\imath_{0}<\cdots<\imath_{n}$ be $n+1$ consecutive elements of $\mathbf{I}$. Define real numbcrs $A_{\imath_{0} .0}, A_{z_{0} .1} \ldots \ldots A_{v_{0} 2 n+1}$ and $B_{\imath_{0}, 0}, B_{\imath_{0}, 1}, \ldots, B_{2_{0}, 2 n+1}$ by the conditions

$$
\begin{aligned}
& X_{10}^{A_{10}{ }^{2 k}}=X_{\imath_{k}}, \\
& X_{\imath_{0}}^{A_{i_{0} 2 k ! 1}}=X_{\imath_{k}+1} \text {, } \\
& X_{\imath_{0}}^{B_{10}{ }^{2 k}}=L_{1 k-1} \text {. } \\
& X_{u_{0}}^{B_{t_{0}}{ }^{2 k+1}}=L_{\imath_{k}}
\end{aligned}
$$

for $k=0, \ldots, n$.

Since $\lambda$ is an exponent of approximation to $\left(1 . \xi . \xi^{3}\right)$. we have

$$
\begin{aligned}
L_{\imath_{0}-1} & \ll X_{\imath_{0}}^{-\lambda} & & \text { by Lemma } 2.3 .1 \\
1 & \ll X_{\imath_{n}}^{-2} L_{\imath_{n}} & & \text { by Lemma } 3.10 \\
1 & \ll X_{\imath_{n}+1} L_{\imath_{n}-1} L_{\imath_{n}} & & \text { by Lemma } 2.3 .2 \\
L_{\imath_{n}} & \ll X_{\imath_{n}-1}^{-\lambda} & & \text { by Lemma } 2.3 .1
\end{aligned}
$$




$$
k=0 \ldots n-1\left\{\begin{aligned}
X_{i_{k}+1} L_{\imath_{k}} & \approx X_{\imath_{k+1}} L_{\imath_{k+1}-1} & & \text { by Corollary } 4.2 . \\
1 & \ll X_{\imath_{k}} X_{\imath_{k+1}} L_{\imath_{k}}^{2} & & \text { by Corollary } 5.5, \\
1 & \ll X_{\imath_{k}+1} L_{\imath_{k}-1} L_{\imath_{k}} & & \text { by Lemma 2.3.2 } \\
L_{\imath_{k}} & \ll X_{l_{k}+1}^{-\lambda} & & \text { by Lemma 2.3.1. }
\end{aligned}\right.
$$

We also note that the condition $X_{\imath_{k}+1} L_{\imath_{k}} \asymp X_{\imath_{k+1}} L_{\imath_{k+1}-1}$ is equivalent to

$$
X_{\imath_{k}+1} L_{\imath_{k}} \ll X_{\imath_{k+1}} L_{\imath_{k+1}-1} \quad \text { and } \quad X_{\imath_{k}-1} L_{\imath_{k}} \gg X_{\imath_{k+1}} L_{\imath_{k+1}-1}
$$

Let $\varepsilon>0$. Assuming that $\iota_{0}$ is sufficiently large, we obtain the following incqualities by passing to the logarithm in base $X_{2_{0}}$ :

$$
\left.\begin{array}{c}
A_{\imath_{0}, 0}=1 \\
B_{\imath_{0}, 0}+\lambda A_{\imath_{0}, 0} \leq \varepsilon \\
-2 A_{\imath_{0}, 2 n}-B_{\imath_{0}, 2 n+1} \leq \varepsilon \\
-A_{\imath_{0}, 2 n+1}-B_{\imath_{0} 2 n}-B_{\imath_{0}, 2 n+1} \leq \varepsilon \\
B_{\imath_{0}, 2 n+1}+\lambda A_{\imath_{0}, 2 n+1} \leq \varepsilon \\
A_{\imath_{0} 2 k+1}+B_{\imath_{0}, 2 k+1}-A_{\imath_{0}, 2 k+2}-B_{\imath_{0}, 2 k+2} \leq \varepsilon \\
-A_{\imath_{0}, 2 k+1}-B_{\imath_{0}, 2 k+1}+A_{\imath_{0}, 2 k+2}+B_{\imath_{0} .2 k+2} \leq \varepsilon \\
-A_{\imath_{0}, 2 k}-A_{\imath_{0}, 2 k+2}-2 B_{\imath_{0}, 2 k+1} \leq \varepsilon \\
-A_{\imath_{0}, 2 k+1}-B_{\imath_{0}, 2 k}-B_{\imath_{0} .2 k+1} \leq \varepsilon \\
B_{\imath_{0}, 2 k+1}+\lambda A_{\imath_{0}, 2 k+1} \leq \varepsilon
\end{array}\right\} k=0, \ldots, n-1
$$

For example, to get the thind inequality, we use $1 \ll X_{\imath_{n}}^{2} L_{n}$. It means that

$$
1 \leq c X_{\iota_{n}}^{2} L_{\imath_{n}}=c X_{\imath_{0}}^{2 A_{u_{0}, 2 n}+B_{u_{0}, 2 n 11}}
$$

for some constant $c$ depending only on $\xi$. Passing to the logar ithm in base $X_{1_{0}}$ we get

$$
0 \leq \log r / \log X_{i_{0}}+2 A_{i_{0}, 2 n}+B_{i_{0}, 2 n+1}
$$


For $i_{0}$ sufficiently large, this gives

$$
0 \leq \varepsilon+2 A_{i_{u}, 2 n}+B_{i_{0}, 2 n+1}
$$

Since for every $\varepsilon>0$ this system of inequalities has a solution, Proposition 6.2 shows that it solution for $\varepsilon=0$.

The question now becomes to determine for which values of $\lambda$, the system of inequalities of Proposition 6.3 has a solution for each integer $n \geq 0$. To analyse this, we first make the following observation.

Lemma 6.4 Let $n \in \mathbb{Z}_{\geq 1}$ and $c>2$. Suppose that $a_{0}, a_{1}, \ldots, a_{n}$ are real numbcrs satisfying the inequalities

$$
a_{k+1} \geq c a_{k}-a_{k-1}, \quad k=1, \ldots, n-1
$$

Then, for any choice of $k, l, m \in \mathbb{Z}_{\geq 0}$ with $k \leq n . m \leq k$ and $l+k \leq n$, we have

$$
c_{m} a_{k+l} \geq c_{k} a_{l+m}-c_{k-m} a_{l}
$$

where the the sequence $\left(c_{i}\right)_{i \geq 0}$ is the solution of the recurrence relation $c_{i+1}=r_{-}-c_{i-1}$ with $c_{1}=1$ and $c_{0}=0$.

Proof: Set $\alpha=\left(c+\sqrt{c^{2}-4}\right) / 2$. The recurrence relation $c_{k+1}=c c_{k}-c_{k-1}$ has $x^{2}-c x+1$ as its companion polynomial with roots $\alpha$ and $\alpha^{-1}$ which are real and distinct since $c>2$. The general solution of the recurrence relation is then $c_{k}=C \alpha^{k}+D \alpha^{-k}$. Solving for $c_{0}=0$ and $c_{1}=1$ we get

$$
c_{k}=\frac{\alpha^{k}-\alpha^{-k}}{\alpha-\alpha^{-1}}
$$

We note that since $c>2$ we have that $\alpha>1$ and so $c_{k} \geq 0$ for all $k \geq 0$. 
We begin hy showing that for $k, l, m \in \mathbb{Z}_{\geq 0}$ with $m \leq k$ and $l+m \leq n-1$ we have

$$
c_{k} a_{l+m+1}-c_{k+1} a_{l+m} \geq c_{k-m} a_{l+1}-c_{k-m+1} a_{l}
$$

We prove this result by induction on $k$. The case $k=0$ is clear. Suppose that for some $k \in \mathbb{Z}_{\geq 0}$ the result holds for each choice of $l . m \in \mathbb{Z}_{\geq 0}$ with $m \leq k$ and $l+m \leq n-1$. Choose $l, m \in \mathbb{Z}_{\geq 0}$ with $m \leq k+1$ and $l+m \leq n-1$. If $m=0$ there is nothing to prove. So we can assume $m>0$. We find

$$
\begin{aligned}
c_{(k+1)} a_{l+m+1}-r_{(k-1)+1} a_{l+m} & \geq c_{(k+1)}\left(c a_{l+m}-a_{l+m-1}\right)-c_{k+2} a_{l+m} & & \text { (hypothesis) } \\
& =c_{k} a_{l+(m-1)+1}-c_{k+1} a_{l+(m-1)} & & \text { (recurrence rel.) } \\
& \geq c_{k-(m-1)} a_{l+1}-c_{k-(m-1)+1} a_{l} & & \text { (induction hyp.) } \\
& =c_{(k+1)-m} a_{l+1}-c_{(k+1)-m+1} a_{l} & &
\end{aligned}
$$

We can now prove the lemma by induction on $k$. The case for $k=0$ is clear. Suppose that for some $k \in \mathbb{Z}_{\geq 0}$ with $k<n$, the result holds for each choice of $l . m \in \mathbb{Z}_{\geq 0}$ with $m \leq k$ and $l+k \leq n$. Choose $l, m \in \mathbb{Z}_{\geq 0}$ with $m \leq k+1$ and $l+k+1 \leq n$. If $m=k+1$ then the result is clear. So we can assume $m<k+1$. Wo find

$$
\begin{array}{rlrl}
c_{m} a_{(h+1)+l} & =c_{m} a_{k+(l+1)} & \\
& \geq c_{h} a_{(l+1)+m}-c_{k-m} a_{l+1} & & \text { (induction hypothesis) } \\
& \geq c_{(k+1)} a_{l+m}-c_{(k+1)-m} a_{l} & & \text { (previous result) }
\end{array}
$$


Theorem 6.5 Under the hypotheses of Proposation 6.3, the system

$$
\begin{gathered}
A_{2} \geq \frac{(\lambda+1)(2 \lambda-1)}{1-\lambda} \\
A_{2 n} \geq c_{n} A_{2}-c_{n-1} \\
1+c_{n-1} A_{2 n} \geq c_{n} \frac{7 \lambda-5}{1-\lambda} A_{2 n}
\end{gathered}
$$

has a solution $\left(A_{2}, A_{2 n}\right) \in \mathbb{R}^{2}$ wuth

$$
c=\frac{2 \lambda-1+\sqrt{\lambda(3 \lambda-2)}}{1-\lambda} \quad \text { and } \quad c_{k}=\frac{\alpha^{k}-\alpha^{-k}}{c-\alpha^{-1}}
$$

Proof: $\quad$ Let $\left(A_{0}, \ldots, A_{2 n+1} . B_{0} \ldots, B_{2 n+1}\right) \in \mathbb{R}^{4 n+4}$ be a solution of the inequalities of Proposition 6.3, with $A_{0}=1$. We claim that $A_{2}$ and $A_{2 n}$ satisfy the conditions of the theorem. To show this we first note that, from (6.7) we have $A_{2 k+1}=A_{2 k+2}+$ $B_{2 h+2}-B_{2 k+1}$ for $k=0, \ldots n-1$. Substituting this into $(6.9)$ and $(6.10)$ we get

$$
\begin{gathered}
A_{2 k+2}+B_{2 k}+B_{2 k+2} \geq 0 \\
(1-\lambda) B_{2 k+1}+\lambda\left(A_{2 k+2}+B_{2 k+2}\right) \leq 0
\end{gathered}
$$

for $h=0 . \ldots n-1$.

From (6.8) we also have $B_{2 k+1} \geq-\left(A_{2 k}+A_{2 k+2}\right) / 2$ for $k=0, \ldots, n-1$. Since $\lambda<1$. substituting this in $(6.13)$ gives

$$
(3 \lambda-1) A_{2 k+2}+2 \lambda B_{2 k+2} \leq(1-\lambda) A_{2 k}
$$

for $k=0 \ldots, n-1$. This inequality can be rewritten in the form

$$
B_{2 k+2} \leq \frac{(1-\lambda)}{2 \lambda} A_{2 k}-\frac{(3 \lambda-1)}{2 \lambda} A_{2 k+2}
$$

for $k=0 . \ldots n-1$.

To eliminate $B_{2 k}$ and $B_{2 k+2}$ from (6.12), we substitute these quantities by the upper bounds given by (6.14). After simplifications, this gives

$$
A_{2 k+2}-\frac{2(2 \lambda-1)}{1-\lambda} A_{2 k}+A_{2 k-2} \geq 0
$$


for $k=1 \ldots n-1$. For $k=0$. we only eliminate $B_{2}$ from (6.12) and get

$$
A_{2} \geq-\frac{(1-\lambda) A_{0}+2 \lambda B_{0}}{1-\lambda}
$$

As (6.3) gives $B_{0} \leq-\lambda A_{0}$. this leads to

$$
A_{2} \geq \frac{(\lambda+1)(2 \lambda-1)}{1-\lambda} A_{0}
$$

Since $A_{0}=1$ this is the first inequality stated in the theorem.

Set $c=2(2 \lambda-1) /(1-\lambda)$. Applying Lemma 6.4 to the inequalities $(6.15)$ for $k=1 \ldots n-1$, with $l=0$ and $m=1$, we obtain

$$
A_{2 k} \geq c_{k} A_{2}-c_{k-1} A_{0}
$$

for $k=1, \ldots n$, where $a$ and $c_{h}$ are given by (6.11). In particular, for $k=n$, we obtain the second inequality stated in the theorem:

$$
A_{2 n} \geq c_{n} A_{2}-c_{n-1} A_{0}
$$

Similarly, applying Lemma 6.4 with $l=0$ and $m=k-1$, we get

$$
A_{0}+c_{k-1} A_{2 k} \geq c_{k} A_{2 k-2}
$$

for $k^{2}=1 \ldots, n$.

By $(6.5)$, we have

$$
A_{2 n+1} \geq-B_{2 n}-B_{2 n+1}
$$

Substituting this in (6.6) we get

$$
(1-\lambda) B_{2 n+1}-\lambda B_{2 n} \leq 0
$$

Combining this with the lower bound for $B_{2 n+1}$ provided by (6.4) and the upper bound for $B_{2 n}$ given by (6.14) for $k=n-1$. we obtain (since $\lambda<1$ ):

$$
A_{2 n-2} \geq \frac{7 \lambda-5}{1-\lambda} A_{2 n}
$$


By relation (6.16) with $k=n$, this finally gives

$$
A_{0}+c_{n-1} A_{2 n} \geq c_{n} \frac{7 \lambda-5}{1-\lambda} A_{2 n}
$$

Corollary 6.6 Let $\xi \in \mathbb{R}$ be such that $1, \xi, \xi^{3}$ are linearly independent over $\mathbb{Q}$. If $\lambda$ is an exponent of approximation to $\left(1, \xi, \xi^{3}\right)$, then we have $\lambda \leq 8 / 11$.

Proof: Suppose on the contrary that $\lambda>8 / 11$. For any $n \in \mathbb{Z}_{\geq 0}$ the theorem provides a solution $\left(A_{2}, A_{2 n}\right) \in \mathbb{R}^{2}$ of the inequalities

$$
\begin{gathered}
A_{2} \geq \frac{(\lambda+1)(2 \lambda-1)}{1-\lambda}, \\
A_{2 n} \geq c_{n} A_{2}-c_{n-1}, \\
1+c_{n-1} A_{2 n} \geq c_{n} \frac{7 \lambda-5}{1-\lambda} A_{2 n},
\end{gathered}
$$

where $\alpha$ and $\left(c_{k}\right)_{k \geq 0}$ are given by $(6.11)$.

Since $\alpha$ and $(\lambda+1)(2 \lambda-1) /(1-\lambda)$ are increasing functions of $\lambda$ for $\lambda \in(2 / 3,1)$, and since $\lambda>8 / 11$, we find that $\alpha>3$ and $(\lambda+1)(2 \lambda-1) /(1-\lambda)>95 / 33$. Thus, the first displayed inequality implies that $A_{2}>95 / 33$. We also note that, since $\alpha>3$, the sequence $\left(c_{k}\right)_{k \geq 1}$ tends to infinity with $k$ and $c_{k}>3 c_{k-1}$ (since $c_{k}-\alpha c_{k-1}=c^{1-k}$ ). In particular, if $n$ is large enough, we obtain

$$
A_{2 n} \geq c_{n} A_{2}-c_{n-1}>c_{n-1}\left(3 A_{2}-1\right)>\frac{84}{33} c_{n-1}
$$

while the last displayed inequality implies

$$
1 \geq c_{n-1}\left(3 \frac{7 \lambda-5}{1-\lambda}-1\right) A_{2 n} .
$$

Combining the two estimates, we get

$$
1 \gg c_{n-1}^{2} \frac{11 \lambda-8}{1-\lambda}
$$


This is a contradiction since $11 \lambda-8>0$.

Remark 6.7 Set $\lambda=8 / 11$. Going back to the proof of Theorem 6.5. a more careful analysis shows that for this $\lambda$, we have for large values of $k$ :

$$
A_{2 k+1} \approx \frac{11}{4} A_{2 k}, \quad A_{2 k+2} \approx 3 A_{2 k} . \quad B_{2 k} \approx-\frac{3}{4} A_{2 k}, \quad B_{2 k+1} \approx-2 A_{2 k} .
$$

For example, this is compatible with the existence of a sequence of minimal points $\left(\mathbf{x}_{i}\right)_{2 \geq 1}$ satisfying the following conditions. For $i<j$ consecutive elements of $\mathbf{I}$, where, as usual $\mathbf{I}$ is set of all indices $i \geq 2$ for which $\mathbf{x}_{\imath-1}, \mathbf{x}_{2}, \mathbf{x}_{n+1}$ are linearly independent, we have:

$$
\begin{array}{cl}
\left\|\mathbf{x}_{\imath}\right\|=X_{\imath}, \quad\left\|\mathbf{x}_{\imath+1}\right\| \asymp X_{\imath}^{1 / 4}, \quad\left\|\mathbf{x}_{\jmath}\right\| \asymp X_{\imath}^{3} . \\
L\left(\mathbf{x}_{\imath}\right) \asymp X_{\imath}^{-2} . \quad L\left(\mathbf{x}_{\jmath-1}\right) \asymp X_{\imath}^{-9 / 4}, \quad L\left(\mathbf{x}_{\jmath}\right) \asymp X_{\imath}^{-6} .
\end{array}
$$




\section{Chapter 7}

\section{The functions $f$ and $g$}

In this chapter, we improve the upper bound on the exponent of approximation from $8 / 11=0 . \overline{72}$ to the root of $4 x^{3}-x^{2}-1$ near 0.7252700 .

Definition 7.1 We set

$$
\begin{gathered}
g(\mathbf{x}, \mathbf{y}, \mathbf{z})=\Phi(\mathbf{x}, \mathbf{y}, \mathbf{z}) \Phi(\mathbf{y}, \mathbf{y}, \mathbf{z})-\Phi(\mathbf{x}, \mathbf{y}, \mathbf{y}) \Phi(\mathbf{y}, \mathbf{z}, \mathbf{z})-\varphi(\mathbf{y}) \Phi(\mathbf{x}, \mathbf{z}, \mathbf{z}) \\
f(\mathbf{y}, \mathbf{z})=g(\mathbf{y}, \mathbf{y}, \mathbf{z})=\Phi(\mathbf{y}, \mathbf{y}, \mathbf{z})^{2}-4 \varphi(\mathbf{y}) \Phi(\mathbf{y} \cdot \mathbf{z}, \mathbf{z})
\end{gathered}
$$

By expanding $g(\mathbf{x}, \mathbf{y}, \mathbf{z})$ in terms of $x_{0}, \Delta x_{1}, \ldots, \Delta z_{2}$ and collecting terms, a simple but tedious computation gives:

$$
\begin{aligned}
|g(\mathbf{x}, \mathbf{y}, \mathbf{z})| \ll & \|\mathbf{y}\|^{3}\|\mathbf{z}\| L(\mathbf{x}) L(\mathbf{z})+\|\mathbf{z}\|^{2} L(\mathbf{x}) L(\mathbf{y})^{3} \\
& +\|\mathbf{x}\|\|\mathbf{y}\|^{3} L(\mathbf{z})^{2}+\|\mathbf{x}\|\|\mathbf{y}\|^{2}\|\mathbf{z}\| L(\mathbf{y}) L(\mathbf{z})
\end{aligned}
$$

In particular, for $f(\mathbf{y}, \mathbf{z})$ we have:

$$
|f(\mathbf{y}, \mathbf{z})| \ll\|\mathbf{y}\|^{3}\|\mathbf{z}\| L(\mathbf{y}) L(\mathbf{z})+\|\mathbf{z}\|^{2} L(\mathbf{y})^{4}+\|\mathbf{y}\|^{4} L(\mathbf{z})^{2}
$$

In the setting of Remark 6.7 , this gives:

$$
g\left(\mathbf{x}_{\imath+1}, \mathbf{x}_{\imath}, \mathbf{x}_{\jmath}\right)=\mathcal{O}\left(X_{\imath}^{-1 / 4}\right) \text { and } f\left(\mathbf{x}_{\imath}, \mathbf{x}_{\jmath}\right)=\mathcal{O}\left(X^{-2}\right)
$$


but since both values are integers we have:

$$
g\left(\mathbf{x}_{i+1}, \mathbf{x}_{i}, \mathbf{x}_{j}\right)=0 \text { and } f\left(\mathbf{x}_{i}, \mathbf{x}_{j}\right)=0
$$

Since $\mathbf{x}_{i+1}$ is a linear combination of $\mathbf{x}_{i}$ and $\mathbf{x}_{j}$, but not a multiple of $\mathbf{x}_{i}$, the following proposition shows that this cannot happen.

Proposition 7.2 Let $\xi \in \mathbb{R}$ be such that $1, \xi, \xi^{3}$ are linearly independent over $\mathbb{Q}$. Let $\left(\mathbf{x}_{i}\right)_{2 \geq 1}$ be a sequence of minimal points associated to $\left(1, \xi, \xi^{3}\right)$. Suppose that $\lambda>2 / 3$ is an exponent of approximation to $\left(1 . \xi, \xi^{3}\right)$. Let $i, j \in \mathbb{Z}_{\geq 1}$ and $\mathbf{x}=a \mathbf{x}_{i}+b \mathbf{x}_{3}$ for some constants $a, b$ with $b \neq 0$. If $i$ and $j$ are sufficiently large, we have $f\left(\mathbf{x}_{i}, \mathbf{x}_{j}\right) \neq 0$ $\operatorname{or} g\left(\mathbf{x}, \mathbf{x}_{i}, \mathbf{x}_{j}\right) \neq 0$.

Proof: Suppose $g\left(\mathbf{x}_{i}, \mathbf{x}_{\imath}, \mathbf{x}_{\jmath}\right)=f\left(\mathbf{x}_{i}, \mathbf{x}_{\jmath}\right)=0$. Then, since $g$ is linear in its first argument we obtain

$$
\begin{aligned}
g\left(\mathbf{x}, \mathbf{x}_{\imath}, \mathbf{x}_{j}\right) & =g\left(a \mathbf{x}_{\imath}+b \mathbf{x}_{j}, \mathbf{x}_{i} \cdot \mathbf{x}_{j}\right)=a g\left(\mathbf{x}_{i}, \mathbf{x}_{\imath} \cdot \mathbf{x}_{\jmath}\right)+b g\left(\mathbf{x}_{j}, \mathbf{x}_{\imath}, \mathbf{x}_{\jmath}\right) \\
& =b g\left(\mathbf{x}_{j} \cdot \mathbf{x}_{i}, \mathbf{x}_{j}\right)=-3 b \varphi\left(\mathbf{x}_{i}\right) \varphi\left(\mathbf{x}_{j}\right) .
\end{aligned}
$$

By Lemma 3.9 this gives $y\left(\mathbf{x}, \mathbf{x}_{i}, \mathbf{x}_{j}\right) \neq 0$ if $i$ and $j$ are sufficiently large.

Let $i<j$ be consecutive elements of $\mathbf{I}$. Our first goal is to show that, if $f\left(\mathbf{x}_{i}, \mathbf{x}_{\jmath}\right) \neq 0$ for arbitrarily large values of $i$, then the exponents of approximation to $\left(1, \xi, \xi^{3}\right)$ are bounded above by $5 / 7$. As a consequence, for an exponent of approximation $\lambda>5 / 7$ we must have $f\left(\mathbf{x}_{i}, \mathbf{x}_{\jmath}\right)=0$, for each sufficiently large $i$. In view of Proposition 7.2 this means that for each $h \in \mathbb{Z}$ with $i<h \leq j$. we have $g\left(\mathbf{x}_{h}, \mathbf{x}_{i}, \mathbf{x}_{j}\right) \neq 0$. This will lead to the main result of this chapter which tells us that exponents of approximation are bounded above by the root of $4 x^{3}-x^{2}-1$ near 0.7252700 . We first establish a technical lemma. 
Lemma 7.3 Let $\xi \in \mathbb{R}$ be such that $1, \xi, \xi^{3}$ are linearly independent over $\mathbb{Q}$. Let $\left(\mathbf{x}_{i}\right)_{2 \geq 1}$ be a sequence of minimal points associated to $\left(1, \xi, \xi^{3}\right)$. Denote by $\mathbf{I}$ the set of all indices $i \geq 2$ for which $\mathbf{x}_{\eta-1}, \mathbf{x}_{i}, \mathbf{x}_{i+1}$ are linearly independent. Suppose that

$$
1 \ll X_{j} L_{\imath}^{2}
$$

for infinitely many pairs of consecutive elements $i<j$ of $\mathbf{I}$. If $\lambda$ is an exponent of approximation to $\left(1 . \xi . \xi^{3}\right)$, then $\lambda \leq 5 / 7$.

Proof: Suppose by contradiction that $\lambda>5 / 7$. We find:

$$
\begin{array}{rlrl}
1 & \ll\left(X_{\jmath} L_{\imath}^{2}\right)^{1-\lambda} & & \text { (hypothesis) } \\
& \ll X_{\jmath}^{1-3 \lambda} L_{j}^{-2(1-\lambda)} & & \text { (Corollary } 4.3) \\
& \ll X_{\jmath}^{1-3 \lambda \cdot-1(1-\lambda)} & & \text { (Lemma 3.10) } \\
& =X_{\jmath}^{-(7 \lambda-5)} . &
\end{array}
$$

This is impossible if $j$ is sufficiently large.

We can now complete our first goal and show that $f\left(\mathbf{x}_{i}, \mathbf{x}_{j}\right)=0$ when $\lambda>5 / 7$.

Lemma 7.4 Let $\xi \in \mathbb{R}$ be such that $1, \xi, \xi^{3}$ are linearly independent over $\mathbb{Q}$. Let $\left(\mathbf{x}_{i}\right)_{i \geq 1}$ be a sequence of minimal points associatcd to $\left(1 . \xi, \xi^{3}\right)$. Denote by $\mathbf{I}$ the set of all indices $i \geq 2$ for which $\mathbf{x}_{1-1}, \mathbf{x}_{i}, \mathbf{x}_{i+1}$ are linearly independent. Suppose that $f\left(\mathbf{x}_{2}, \mathbf{x}_{l}\right) \neq 0$ for infinitely many pairs of consecutive elements $i<j$ of $\mathbf{I}$. If $\lambda$ is an exponent of approximation to $\left(1, \xi, \xi^{3}\right)$, then $\lambda \leq 5 / 7$.

Proof: Suppose by contradiction that $\lambda>5 / 7$. Using the expansion of $f(\mathbf{x}, \mathbf{y})$ given by (7.2) we get

$$
1 \leq\left|f\left(\mathbf{x}_{i} \cdot \mathbf{x}_{\jmath}\right)\right| \ll X_{i}^{3} X_{j} L_{\imath} L_{j}+X_{j}^{2} L_{i}^{4}
$$


If $1 \ll X_{j}^{-2} L_{i}^{4}$, then we also have $1 \ll X_{j} L_{i}^{2}$ in cont radiction Lemma 7.3. Therefore we must have $1 \ll X_{i}^{3} X_{j} L_{l} L_{j}$. We will show that this is impossible if $\lambda>(5-\sqrt{13}) / 2$. Indeed, we have:

$$
\begin{aligned}
1 & \ll\left(X_{i}^{3} X_{j} L_{i} L_{j}\right)^{\lambda(1-\lambda)} & & \text { (hypothesis) } \\
& \ll X_{i}^{3 \lambda(1-\lambda)} X_{j}^{-\lambda(2 \lambda-1)} & & \text { (Corollary 4.3) } \\
& \ll X_{i+1}^{3(1-\lambda)^{2}} X_{j}^{-\lambda(2 \lambda-1)} & & (\text { Lemma 2.3.4) } \\
& \leq X_{j}^{\lambda^{2}-5 \lambda+3} & & \left(X_{i+1} \leq X_{j}\right)
\end{aligned}
$$

which is a contradiction for $j$ sufficiently large since $\lambda^{2}-5 \lambda+3$ is negative for any $\lambda$ in the interval from $(5-\sqrt{13}) / 2 \approx 0.697224$ to 1 .

Corollary 7.5 Let $\xi \in \mathbb{R}$ be such that $1, \xi, \xi^{3}$ are linearly independent over $\mathbb{Q}$. Let $\left(\mathbf{x}_{i}\right)_{2 \geq 1}$ be a sequence of minimal points associated to $\left(1, \xi, \xi^{3}\right)$. Denote by $\mathbf{I}$ the set of all indices $i \geq 2$ for which $\mathbf{x}_{i-1}, \mathbf{x}_{i}, \mathbf{x}_{i+1}$ are linearly independent. Suppose that $\lambda>5 / 7$ is an cxponent of approximation to $\left(1, \xi . \xi^{3}\right)$. Then for all pairs of consecutive elcments $i<j$ of $\mathbf{I}$ with $i$ sufficiently large we have $f\left(\mathbf{x}_{i}, \mathbf{x}_{j}\right)=0$.

We now look at what happens when $f\left(\mathbf{x}_{i}, \mathbf{x}_{j}\right)=0$ for all sufficiently large $i$.

Lemma 7.6 Let $\xi \in \mathbb{R}$ be such that $1, \xi, \xi^{3}$ are linearly independent over $\mathbb{Q}$. Let $\left(\mathbf{x}_{i}\right)_{1 \geq 1}$ be a sequence of minimal points associated to $\left(1, \xi, \xi^{3}\right)$. Denote by $\mathbf{I}$ the set of all indices $i \geq 2$ for which $\mathbf{x}_{1-1}, \mathbf{x}_{i}, \mathbf{x}_{2+1}$ are linearly indepcndcnt. Suppose that $\lambda>2 / 3$ is an exponent of approximation to $\left(1, \xi, \xi^{3}\right)$. Then for any pair of consccutive elements $i<j$ of $\mathbf{I}$ with $f\left(\mathbf{x}_{i}, \mathbf{x}_{j}\right)=0$ we have

$$
1 \ll X_{j}^{2} L_{i}^{4}+X_{i}^{2} X_{i+1} X_{j} L_{i} L_{j}
$$


Proof: By Proposition 7.2 we have $g\left(\mathbf{x}_{i+1}, \mathbf{x}_{i}, \mathbf{x}_{j}\right) \neq 0$. Combining this with the expansion of $g(\mathbf{x}, \mathbf{y}, \mathbf{z})$ given by (7.1) we find that

$$
1 \leq\left|g\left(\mathbf{x}_{\imath+1}, \mathbf{x}_{i}, \mathbf{x}_{\jmath}\right)\right| \ll X_{j}^{2} L_{i}^{3} L_{i+1}+X_{i}^{2} X_{i+1} X_{j} L_{\imath} L_{j} \leq X_{j}^{2} L_{i}^{4}+X_{\imath}^{2} X_{\imath+1} X_{j} L_{i} L_{j}
$$

Note that in the case where $\lambda>5 / 7$ only the second term in the RHS of the inequality is of interest. Since $X_{i+1} \leq X_{h}$ for $h=i+1, \ldots j$, the choice of $h=i+1$ in the preceding argument provides us with the smallest upper bound for $\left|g\left(\mathbf{x}_{h} . \mathbf{x}_{i}, \mathbf{x}_{j}\right)\right|$.

Corollary 7.7 Let $\xi \in \mathbb{R}$ be such that $1, \xi, \xi^{3}$ are linearly independent over $\mathbb{Q}$. Let $\left(\mathbf{x}_{i}\right)_{\imath \geq 1}$ be a sequence of minimal points associated to $\left(1, \xi, \xi^{3}\right)$. Dcnote by $\mathbf{I}$ the set of all indices $i \geq 2$ for which $\mathbf{x}_{i-1}, \mathbf{x}_{i}, \mathbf{x}_{i+1}$ are linearly independent. Suppose that $\lambda>5 / 7$ is an exponent of approximation $t o\left(1, \xi, \xi^{3}\right)$. Then for any pair of consecutive elements $i<j$ of $\mathbf{I}$ we have

$$
1 \ll X_{i}^{2} X_{1+1} X_{j} L_{\imath} L_{j}
$$

Proof: By Corollary 7.5 we have that $f\left(\mathbf{x}_{i}, \mathbf{x}_{j}\right)=0$ and by Lemma 7.6 it follows that

$$
1 \ll X_{j}^{2} L_{i}^{4}+X_{\imath}^{2} X_{i+1} X_{j} L_{i} L_{j}
$$

The conclusion follows from Lemma 7.3 since the summand $X_{j}^{2} L_{\imath}^{4} \rightarrow 0$ as $i \rightarrow \infty$.

Theorem 7.8 Let $\xi \in \mathbb{R}$ be such that $1, \xi, \xi^{3}$ are linearly independent over $\mathbb{Q}$. If $\lambda$ is an exponent of approximation to $\left(1, \xi, \xi^{3}\right)$, then $\lambda \leq \lambda_{0}$ where $\lambda_{0}$ is the root of $4 x^{3}-x^{2}-1$ neur 0.7252700 . 
Proof: Let $\left(\mathbf{x}_{\imath}\right)_{l \geq 1}$ be a sequence of minimal points associated to $\left(1, \xi, \xi^{3}\right)$. Denote by $\mathbf{I}$ the set of all indices $i \geq 2$ for which $\mathbf{x}_{2-1}, \mathbf{x}_{\imath}, \mathbf{x}_{2+1}$ are linearly independent. Let $i<j$ be a pair of consecntive elements of $\mathbf{I}$ with $i$ arbitrarily large. Suppose by contradiction that $\lambda>\lambda_{0}$. We then get

$$
\begin{aligned}
1 & \ll\left(X_{\imath}^{2} X_{\imath+1} X_{\jmath} L_{\imath} L_{\jmath}\right)^{\lambda(1-\lambda)} \\
& \ll\left(X_{\imath}^{2} X_{\imath+1}\right)^{\lambda(1-\lambda)} X_{\jmath}^{-\lambda(2 \lambda-1)} \\
& \ll\left(X_{\imath}^{2} X_{\imath+1}\right)^{\lambda(1-\lambda)}\left(X_{\imath} L_{\imath}^{2}\right)^{\lambda(2 \lambda-1)} \\
& \ll X_{\imath+1}^{(1-\lambda)(1-\lambda)} L_{\imath}^{2 \lambda(2 \lambda-1)} \\
& \ll X_{\imath+1}^{-\left(4 \lambda^{3}-\lambda^{2}-1\right)}
\end{aligned}
$$

which is a impossible for $i$ sufficiently large since $4 \lambda^{3}-\lambda^{2}-1>0$.

Note that we can improve on this upper bound for $\lambda$ using an argument similar to the one presented in Chapter 6 to prove $\lambda \leq 8 / 11$. To this end we replace the condition $0 \leq 2 A_{2 n}+B_{2 n+1}$ with $0 \leq 2 A_{2 n-2}+A_{2 n-1}+A_{2 n}+B_{2 n-1}+B_{2 n+1}$ in Proposition 6.3. In this way we find that, if $\lambda$ is an exponent of approximation to $\left(1 . \xi . \xi^{3}\right)$, then $\lambda \leq \lambda_{0}$ where $\lambda_{0}$ is the root of $16 x^{3}-7 x-1$ near 0.7237766 . 


\section{Chapter 8}

\section{The point $S$ and estimates for $g$}

In this chapter. we first note that the upper bound for an exponent of approximation to $\left(1, \xi, \xi^{3}\right)$ found in Chapter 7 is not optimal. This is done by constructing various new points which must be zero for an exponent of approximation $\lambda>0.72377 \ldots$, the value found at the end of Chapter 7 . We also introrluce a new point $S$ which will play an important role in the sequel. Finally, we provide estimates for $g\left(\mathbf{x}_{i-1}, \mathbf{x}_{1}, \mathbf{x}_{3}\right)$ when $i<j$ are consecutive elements of $\mathbf{I}$ and conclude that these values are 0 when $\left(1, \xi, \xi^{3}\right)$ admits an exponent of approximation $\lambda>5 / 7$.

\subsection{Various new points}

Definition 8.1.1 We set

$$
\tilde{\psi}(\mathbf{x}, \mathbf{y}):=\Phi(\mathbf{x}, \mathbf{x}, \mathbf{y}) \mathbf{y}-2 \Phi(\mathbf{x}, \mathbf{y}, \mathbf{y}) \mathbf{x}
$$

and let $\wedge$ denote the usual cross product

$$
\mathbf{x} \wedge \mathbf{y}:=\left(\left|\begin{array}{ll}
x_{1} & x_{2} \\
y_{1} & y_{2}
\end{array}\right|,-\left|\begin{array}{ll}
x_{0} & x_{2} \\
y_{0} & y_{2}
\end{array}\right|,\left|\begin{array}{ll}
x_{0} & x_{1} \\
y_{0} & y_{1}
\end{array}\right|\right)
$$

where $\mathbf{x}=\left(x_{0}, x_{1}, x_{2}\right)$ and $\mathbf{y}=\left(y_{0}, y_{1}, y_{2}\right)$. 
Note that we have

$$
\psi(\mathbf{x}, \mathbf{y}) \wedge \tilde{\psi}(\mathbf{x}, \mathbf{y})=f(\mathbf{x}, \mathbf{y}) \mathbf{x} \wedge \mathbf{y}
$$

which means that $\psi(\mathbf{x}, \mathbf{y}) \propto \tilde{\psi}(\mathbf{x}, \mathbf{y})$ when $f(\mathbf{x}, \mathbf{y})=0$.

Definition 8.1.2 For any $\mathbf{x}=\left(x_{0}, x_{1}, x_{2}\right)$ and $\mathbf{y}=\left(y_{0}, y_{1}, y_{2}\right)$, we also define

$$
\begin{aligned}
& \theta_{0}(\mathbf{x}, \mathbf{y}):=y_{0} \psi(\mathbf{x}, \mathbf{y})+x_{0} \tilde{\psi}(\mathbf{x}, \mathbf{y}) \\
& \theta_{1}(\mathbf{x}, \mathbf{y}):=y_{1} \psi(\mathbf{x}, \mathbf{y})+x_{1} \tilde{\psi}(\mathbf{x}, \mathbf{y}) \\
& \theta_{2}(\mathbf{x}, \mathbf{y}):=y_{2} \psi(\mathbf{x}, \mathbf{y})+x_{2} \tilde{\psi}(\mathbf{x}, \mathbf{y})
\end{aligned}
$$

We can also write these points as

$$
\begin{aligned}
& \theta_{0}(\mathbf{x}, \mathbf{y})=\psi(\mathbf{x}, \mathbf{y})_{0} \mathbf{y}+\tilde{\psi}(\mathbf{x}, \mathbf{y})_{0} \mathbf{x} \\
& \theta_{\mathbf{1}}(\mathbf{x}, \mathbf{y})=\psi(\mathbf{x}, \mathbf{y})_{1} \mathbf{y}+\tilde{\psi}(\mathbf{x}, \mathbf{y})_{1} \mathbf{x} \\
& \theta_{2}(\mathbf{x}, \mathbf{y})=\dot{\psi}(\mathbf{x}, \mathbf{y})_{2} \mathbf{y}+\tilde{\psi}(\mathbf{x}, \mathbf{y})_{2} \mathbf{x} .
\end{aligned}
$$

It is interesting to note that

$$
2 g(\mathbf{x}, \mathbf{y}, \mathbf{z})=\theta_{0}(\mathbf{y}, \mathbf{z})_{0} x_{2}-3 \theta_{1}(\mathbf{y}, \mathbf{z})_{1} x_{1}+2 \theta_{0}(\mathbf{y}, \mathbf{z})_{2} x_{0}
$$

which can also be written as

$$
2 g(\mathbf{x}, \mathbf{y}, \mathbf{z})=\Phi(\mathbf{x}, \mathbf{z}, \psi(\mathbf{y}, \mathbf{z}))+\Phi(\mathbf{x}, \mathbf{y}, \tilde{\psi}(\mathbf{y}, \mathbf{z}))
$$

Using the points $\theta_{i}$, we construct further new points:

$$
\begin{gathered}
2 x_{i} g(\mathbf{x}, \mathbf{y}, \mathbf{z}) \mathbf{x}-\varphi(\mathbf{x}) \theta_{i}(\mathbf{y}, \mathbf{z}), \quad i=0,1,2 \\
4 x_{i} g(\mathbf{x}, \mathbf{y}, \mathbf{z}) \mathbf{x} \wedge \mathbf{y}+\theta_{i}(\mathbf{y}, \mathbf{z}) \wedge \psi(\mathbf{x}, \mathbf{y}), \quad i=0,1,2 \\
f(\mathbf{y}, \mathbf{z}) \psi(\mathbf{x}, \mathbf{z})+4 \varphi(\mathbf{y}) \varphi(\mathbf{z}) \psi(\mathbf{x}, \mathbf{y})
\end{gathered}
$$

For the exponent $\lambda=0.7237766$, it can be shown that all these points vanish when evaluated at $(\mathbf{x}, \mathbf{y}, \mathbf{z})=\left(\mathbf{x}_{i}, \mathbf{x}_{j}, \mathbf{x}_{k}\right)$ where $i<j<k$ are consecutive elements of $\mathbf{I}$ with 
l sufficiently large. Right away we note that since $\lambda>5 / 7$ we have $f\left(\mathbf{x}_{j}, \mathbf{x}_{h}\right)=0$ and so the last point is non-zero. This is a contradiction. More precisely, we find the following uppes bound for the norm of this point:

$$
\begin{aligned}
& \left\|\psi(\mathbf{x}, \mathbf{z}) f(\mathbf{y}, \mathbf{z})+4 \psi^{\prime}(\mathbf{x}, \mathbf{y}) \varphi(\mathbf{y}) \varphi(\mathbf{z})\right\| \\
& \ll\|\mathbf{x}\|^{3}\|\mathbf{y}\|^{4} L(\mathbf{z})^{3}+\|\mathbf{x}\|\|\mathbf{y}\|^{4}\|\mathbf{z}\| L(\mathbf{x})^{2} L(\mathbf{z})^{2}+\|\mathbf{x}\|^{3}\|\mathbf{y}\|^{3}\|\mathbf{z}\| L(\mathbf{y}) L(\mathbf{z})^{2} \\
& \quad+\|\mathbf{x}\|^{3}\|\mathbf{y}\|^{2}\|\mathbf{z}\|^{2} L(\mathbf{y})^{2} L(\mathbf{z})+\|\mathbf{x}\|\|\mathbf{z}\|^{3} L(\mathbf{x})^{2} L(\mathbf{y})^{1}
\end{aligned}
$$

If $\imath<\jmath<k$ are consecutive elements of $\mathbf{I}$ with $f\left(\mathbf{x}_{\jmath}, \mathbf{x}_{h}\right)=0$ and $\imath$ sufficiently large then we have

$$
f\left(\mathbf{x}_{\jmath}, \mathbf{x}_{h}\right) \psi\left(\mathbf{x}_{i}, \mathbf{x}_{h}\right)+4 \varphi\left(\mathbf{x}_{\jmath}\right) \varphi\left(\mathbf{x}_{k}\right) \psi\left(\mathbf{x}_{\imath}, \mathbf{x}_{\jmath}\right) \neq 0
$$

and so the above estimate applied to the norm of this non-zero integer point gives:

$$
1 \ll X_{\imath}^{3} X_{\jmath}^{2} X_{k}^{2} L_{\jmath}^{2} L_{k}+X_{\imath} X_{k}^{3} L_{\imath}^{2} L_{\jmath}^{4}
$$

Using this we can show that, if $\lambda$ is an exponent of approximation, then $\lambda \leq 072211$. We will not provide a proof for this. but proceed to look at a point which will later be used to prove stronger results.

\subsection{The point $S$}

Definition 8.2.1 We set

$$
\begin{aligned}
S(\mathbf{x} . \mathbf{y}, \mathbf{z})= & (\Phi(\mathbf{x}, \mathbf{x} \cdot \mathbf{y}) \Phi(\mathbf{x} \cdot \mathbf{x}, \mathbf{z})-4 \varphi(\mathbf{x}) \Phi(\mathbf{x}, \mathbf{y} \cdot \mathbf{z})) \mathbf{x} \\
& -2 \varphi(\mathbf{x}) \Phi(\mathbf{x} \cdot \mathbf{x}, \mathbf{z}) \mathbf{y}+2 \varphi(\mathbf{x}) \Phi(\mathbf{x}, \mathbf{x}, \mathbf{y}) \mathbf{z}
\end{aligned}
$$

Note that $S$ satisfies

$$
S(\mathbf{x}, \mathbf{x}, \mathbf{y})=-3 \varphi(\mathbf{x}) \psi^{\prime}(\mathbf{x}, \mathbf{y}) \text { and } S(\mathbf{x}, \mathbf{y}, \mathbf{y})=f(\mathbf{x} \cdot \mathbf{y}) \mathbf{x}
$$


By expanding $S(\mathbf{x}, \mathbf{y}, \mathbf{z})$ in terms of $x_{0}, \Delta x_{1}, \ldots, \Delta z_{2}$, we find the following upper bound for the norm of this point:

$$
\begin{aligned}
\|S(\mathbf{x}, \mathbf{y}, \mathbf{z})\| \ll & \|\mathbf{x}\|^{5} L(\mathbf{y}) L(\mathbf{z})+\|\mathbf{x}\|^{4}\|\mathbf{y}\| L(\mathbf{x}) L(\mathbf{z}) \\
& +\|\mathbf{x}\|^{3}\|\mathbf{z}\| L(\mathbf{x})^{2} L(\mathbf{y})+\|\mathbf{x}\|\|\mathbf{y}\|\|\mathbf{z}\| L(\mathbf{x})^{4}
\end{aligned}
$$

Proposition 8.2.2 Let $\xi \in \mathbb{R}$ be such that $1, \xi . \xi^{3}$ are linearly independent, over $\mathbb{Q}$. Lct $\left(\mathbf{x}_{i}\right)_{i \geq 1}$ be a sequence of minimal points associated to $\left(1, \xi, \xi^{3}\right)$. Suppose that $\lambda>2 / 3$ is an exponent of approximation to $\left(1, \xi, \xi^{3}\right)$. Let $i, j \in \mathbb{Z}_{\geq 1}$ and $\mathbf{x}=a \mathbf{x}_{1}+b \mathbf{x}_{\text {, }}$ for some constants $a, b$ with $a \neq 0$. Then of $i$ and $j$ are sufficiently lurge we have $f\left(\mathbf{x}_{i}, \mathbf{x}_{j}\right) \neq 0$ or $S\left(\mathbf{x}_{\imath}, \mathbf{x}, \mathbf{x}_{\jmath}\right) \neq 0$.

Proof: Suppose $f\left(\mathbf{x}_{i}, \mathbf{x}_{j}\right)=0$. Then, since $S$ is linear in its second argument we obtain

$$
\begin{aligned}
S\left(\mathbf{x}_{i}, \mathbf{x}, \mathbf{x}_{j}\right)=S\left(\mathbf{x}_{i}, a \mathbf{x}_{i}+b \mathbf{x}_{j}, \mathbf{x}_{j}\right) & =-3 a \varphi\left(\mathbf{x}_{\imath}\right) \psi\left(\mathbf{x}_{i}, \mathbf{x}_{\jmath}\right)+b f\left(\mathbf{x}_{\imath}, \mathbf{x}_{j}\right) \mathbf{x}_{i} \\
& =-3 a \varphi\left(\mathbf{x}_{i}\right) \psi\left(\mathbf{x}_{i}, \mathbf{x}_{j}\right)
\end{aligned}
$$

By Lemmas 3.9 and 5.4 this implies that $S\left(\mathbf{x}_{i}, \mathbf{x}, \mathbf{x}_{j}\right) \neq 0$ if $i$ and $j$ are sufficiently large.

Lemma 8.2.3 Let $\xi \in \mathbb{R}$ be such that $1, \xi, \xi^{3}$ are linearly independent over $\mathbb{Q}$. Let $\left(\mathbf{x}_{i}\right)_{i \geq 1}$ be a sequence of minimal points associated to $\left(1, \xi, \xi^{3}\right)$. Denote by $\mathbf{I}$ the set of all indices $i \geq 2$ for which $\mathbf{x}_{i-1}, \mathbf{x}_{i}, \mathbf{x}_{i+1}$ are linearly independent. Suppose that $\lambda>2 / 3$ is an exponcnt of approximation to $\left(1, \xi, \xi^{3}\right)$. Let $i<j$ be a pair of elements of $\mathbf{I}$ for which $f\left(\mathbf{x}_{i}, \mathbf{x}_{j}\right)=0$. Then we have

$$
1 \ll X_{i}^{4} X_{j} L_{i} L_{j}+X_{i}^{3} X_{i+1} L_{i}^{3}+X_{i} X_{j}^{-2} L_{i}^{4}
$$

Proof: Since $\mathbf{x}_{j-1}$ is a linear combination of $\mathbf{x}_{i}$ and $\mathbf{x}_{j}$, but not a multiple of $\mathbf{x}_{j}$. Proposition 8.2.2 shows that $S\left(\mathbf{x}_{\imath}, \mathbf{x}_{j-1}, \mathbf{x}_{j}\right)$ is a non-zero point of $\mathbb{Z}^{3}$. So its norm is 
bounded below by 1 . On the other hand, using the general upper bound for the norm of $S$ given above we find:

$$
\begin{aligned}
\left\|S\left(\mathbf{x}_{i}, \mathbf{x}_{\jmath-1}, \mathbf{x}_{j}\right)\right\| & \ll X_{i}^{5} L_{j-1} L_{j}+X_{i}^{4} X_{\jmath-1} L_{\imath} L_{\jmath}+X_{i}^{3} X_{\jmath} L_{i}^{2} L_{j-1}+X_{\imath} X_{\jmath-1} X_{\jmath} L_{\imath}^{4} \\
& \ll X_{\imath}^{4} X_{j-1} L_{i} L_{\jmath}+X_{i}^{3} X_{j} L_{\imath}^{2} L_{j-1}+X_{\imath} X_{\jmath-1} X_{j} L_{\imath}^{4} \\
& \ll X_{\imath}^{4} X_{j} L_{i} L_{j}+X_{i}^{3} X_{\jmath} L_{\imath}^{2} L_{\jmath-1}+X_{\imath} X_{j}^{2} L_{\imath}^{4} \\
& \asymp X_{\imath}^{4} X_{\jmath} L_{\imath} L_{j}+X_{i}^{3} X_{\imath+1} L_{\imath}^{3}+X_{i} X_{j}^{2} L_{\imath}^{4}
\end{aligned}
$$

where the last equivalence comes from Corollary 4.3.

\subsection{New estimates for $g$}

Since $g$ is linear in its first argument, we can view it as a scalar product.

Definition 8.3.1 For $\mathbf{x}=\left(x_{0}, x_{1}, x_{2}\right)$ and $\mathbf{y}=\left(y_{0}, y_{1}, y_{2}\right)$ we define the scular product by

$$
\langle\mathbf{x} \cdot \mathbf{y}\rangle=x_{0} y_{0}+x_{1} y_{1}+x_{2} y_{2}
$$

Identifying polynomials $a+b T+c T^{3} \in \mathbb{R}[T]$ with points $(a, b, c) \in \mathbb{R}^{3}$, we also define. a polynomial $P_{\mathbf{y z}} \in \mathbb{R}[\mathbf{y}, \mathbf{z}][T]$ by

$$
\left\langle\mathbf{x}, P_{\mathbf{y z}}\right\rangle=g(\mathbf{x}, \mathbf{y}, \mathbf{z}) .
$$

Lemma 8.3.2 Let $\mathbf{y}, \mathbf{z} \in \mathbb{Z}^{3}$ with $\varphi(\mathbf{y}) \neq 0$ and $f(\mathbf{y} \cdot \mathbf{z})=0$. Then we have

$$
\left\|P_{\mathbf{y z}}\right\| \ll\|\psi \cdot(\mathbf{y}, \mathbf{z})\|^{2} \quad \text { and } \quad\left|P_{\mathbf{y z}}(\xi)\right| \ll\left\|P_{\mathbf{y} \mathbf{z}}\right\| \frac{L(\mathbf{y})}{\|\mathbf{y}\|} \text {. }
$$

Moreover, for any $\mathbf{x} \in \mathbb{Z}^{3}$, we also have

$$
|g(\mathbf{x}, \mathbf{y}, \mathbf{z})| \ll\left(\|\mathbf{x}\|\|\mathbf{y}\|^{-1} L(\mathbf{y})+L(\mathbf{x})\right)\|\psi(\mathbf{y}, \mathbf{z})\|^{2}
$$


Proof: $\Lambda$ computation shows the identity

$$
\Phi\left(\mathbf{x}, \iota^{\prime}(\mathbf{y}, \mathbf{z}), \psi(\mathbf{y}, \mathbf{z})\right)=\Phi(\mathbf{x}, \mathbf{y} \cdot \mathbf{y}) f(\mathbf{y}, \mathbf{z})-4 \varphi(\mathbf{y}) g(\mathbf{x}, \mathbf{y} \cdot \mathbf{z})
$$

and also that

$$
\Phi(\mathbf{x}, \mathbf{y}, \mathbf{y})=2 x_{0} y_{0} y_{2}-3 x_{1} y_{1}^{2}+x_{2} y_{0}^{2} .
$$

Since $f(\mathbf{y}, \mathbf{z})=0$, the first formula reduces to

$$
-4 \varphi(\mathbf{y}) g(\mathbf{x}, \mathbf{y} \cdot \mathbf{z})=2 x_{0} \psi_{0} \psi_{2}-3 x_{1} \psi_{1}^{2}+x_{2} v_{0}^{2}
$$

where $\psi^{\prime}=v(\mathbf{y}, \mathbf{z})$. Since this holds for any $\mathbf{x}$. we conclude that

$$
-4 \varphi(\mathbf{y}) P_{\mathbf{y z}}=2 \gamma_{0}^{\prime} \psi_{2}-3 T w_{1}^{2}+T^{3} \imath^{\prime}{ }_{0}^{2}
$$

Since $\rho(\mathbf{y})$ is a non-zelo integer, we have $|\varphi(y)| \geq 1$ and so the above formula learts to

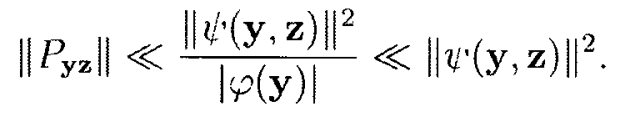

We also note that

$$
0=f(\mathbf{y} \cdot \mathbf{z})=g(\mathbf{y} \cdot \mathbf{y}, \mathbf{z})=\left\langle\mathbf{y}, P_{\mathbf{y z}}\right\rangle=\left\langle y_{0} \underline{\underline{\xi}}+\Delta \mathbf{y} \cdot P_{\mathbf{y z}}\right\rangle=y_{0} P_{\mathbf{y z}}(\xi)+\left\langle\Delta \mathbf{y} \cdot P_{\mathbf{y z}}\right\rangle
$$

and so

$$
\left|P_{\mathbf{y z}}(\xi)\right|=\frac{\left|\left\langle\Delta \mathbf{y}, P_{\mathbf{y z}}\right\rangle\right|}{\left|y_{0}\right|} \ll\left\|P_{\mathrm{yz}}\right\| \frac{L(\mathbf{y})}{\|\mathbf{y}\|} .
$$

This proves (8.3.1). For $\mathbf{x} \in \mathbb{Z}^{3}$. we deduce that

$$
\begin{aligned}
|g(\mathbf{x} . \mathbf{y}, \mathbf{z})| & =\left|\left\langle\mathbf{x}, P_{\mathbf{y} \mathbf{z}}\right\rangle\right|=\left|x_{0} P_{\mathbf{y z}}(\xi)+\left\langle\Delta \mathbf{x}, P_{\mathbf{y} \mathbf{z}}\right\rangle\right| \\
& \ll\|\mathbf{x}\|\left|P_{\mathbf{y} \mathbf{z}}(\xi)\right|+\left\|P_{\mathbf{y z}}\right\| L(\mathbf{x}) \\
& \ll\left(\|\mathbf{x}\|\|\mathbf{y}\|^{-1} L(\mathbf{y})+L(\mathbf{x})\right)\left\|P_{\mathbf{y z}}\right\| \\
& \ll\left(\|\mathbf{x}\|\|\mathbf{y}\|^{-1} L(\mathbf{y})+L(\mathbf{x})\right)\|\psi(\mathbf{y}, \mathbf{z})\|^{2}
\end{aligned}
$$

We will need the following technical lemma which refines Lemma 7.3. 
Lemma 8.3.3 Let $\xi \in \mathbb{R}$ be such that $1, \xi, \xi^{3}$ are linearly independent over $\mathbb{Q}$. Let $\left(\mathbf{x}_{\imath}\right)_{i \geq 1}$ be a sequence of minimal points associated to $\left(1, \xi, \xi^{3}\right)$. Denote by $\mathbf{I}$ the set of all indices $i \geq 2$ for which $\mathbf{x}_{i-1}, \mathbf{x}_{i}, \mathbf{x}_{1+1}$ are linearly independent. Suppose that

$$
1 \ll X_{i} X_{j}^{2} L_{i}^{4}
$$

for infinitely many pairs of consecutive elements $i<j$ of $\mathbf{I}$. If $\lambda$ is an exponent of approximation to $\left(1, \xi . \xi^{3}\right)$, then $\lambda \leq 5 / 7$.

Proof: Suppose by contradiction that $\lambda>5 / 7$. We then find:

$$
\begin{aligned}
1 & \ll\left(X_{i} X_{\jmath}^{2} L_{i}^{4}\right)^{\lambda} & & \text { (hypothesis) } \\
& \ll\left(X_{i} X_{j}^{2} X_{\imath+1}^{-4 \lambda}\right)^{\lambda} & & \text { (Lemma 2.3.1) } \\
& \ll X_{\imath+1}^{1-\lambda-4 \lambda^{2}} X_{\jmath}^{2 \lambda} & & \text { (Lemma 2.3.4) }
\end{aligned}
$$

However we also have:

$$
\begin{aligned}
1 & \ll\left(X_{\imath}^{2} X_{\imath+1} X_{j} L_{i} L_{j}\right)^{\lambda(1-\lambda)} \\
& \ll\left(X_{\imath}^{2} X_{1+1}\right)^{\lambda(1-\lambda)} X_{j}^{-\lambda(2 \lambda-1)} \\
& \ll X_{i+1}^{(2-\lambda)(1-\lambda)} X_{j}^{-\lambda(2 \lambda-1)}
\end{aligned}
$$

Combining these two results we obtain

$$
1 \ll X_{i+1}^{-\left(8 \lambda^{3}-4 \lambda^{2}+3 \lambda-3\right)}
$$

which is a contradiction if $i$ sufficiently large since $8 \lambda^{3}-4 \lambda^{2}+3 \lambda-3>0$ when $\lambda>\lambda_{0}$, where $\lambda_{0}$ is the real root of $8 x^{3}-4 x^{2}+3 x-3$ near 0.7124456 .

Lemma 8.3.4 Let $\xi \in \mathbb{R}$ be such that $1, \xi, \xi^{3}$ are linearly independent over $\mathbb{Q}$. Let $\left(\mathbf{x}_{i}\right)_{i \geq 1}$ be a sequence of minimal points associated to $\left(1 . \xi, \xi^{3}\right)$. Denote by $\mathbf{I}$ the set of all indices $i \geq 2$ for which $\mathbf{x}_{i-1}, \mathbf{x}_{i}, \mathbf{x}_{i+1}$ are linearly independent. Suppose that, for 
infinately many pairs of consecutive elements $\imath<\jmath$ of $\mathbf{I}$ we have $g\left(\mathbf{x}_{\imath-1}, \mathbf{x}_{\imath}, \mathbf{x}_{j}\right) \neq 0$. If $\lambda$ is an exponent of approxmation to $\left(1, \xi, \xi^{3}\right)$, then $\lambda \leq 5 / 7$.

Proof: Suppose by contradiction that $\lambda>5 / 7$. Choose a triple $i<\jmath<k$ of consecutive elements of $\mathbf{I}$ with $\iota$ arbitrarily large such that $g\left(\mathbf{x}_{\imath-1}, \mathbf{x}_{\imath}, \mathbf{x}_{\jmath}\right) \neq 0$. Then we find

$$
\begin{aligned}
1 & \leq\left|g\left(\mathbf{x}_{\imath-1} \cdot \mathbf{x}_{\imath}, \mathbf{x}_{\jmath}\right)\right| & & \text { (hypothesis) } \\
& \ll\left(X_{\imath-1} X_{\imath}^{-1} L_{\imath}+L_{\imath-1}\right)\left\|\psi\left(\mathbf{x}_{\imath}, \mathbf{x}_{\jmath}\right)\right\|^{2} & & \text { (Lemma 8.3.2) } \\
& \ll L_{\imath-1}\left\|\imath_{\imath}\left(\mathbf{x}_{\imath}, \mathbf{x}_{\jmath}\right)\right\|^{2} & & \left(X_{\imath-1} \leq X_{\imath}, L_{\imath} \leq L_{\imath-1}\right) \\
& \ll X_{\imath}^{2} X_{\imath}^{2} L_{l-1} L_{\imath}^{4} & & \text { (Lemma 5.3). }
\end{aligned}
$$

We first claim that $X_{\imath \nmid 1}^{3} \ll X_{j+1}$. To show this, we observe that

$$
\begin{aligned}
1 & \ll\left(X_{\imath}^{2} X_{\jmath}^{2} L_{\imath-1} L_{\imath}^{4}\right)^{\lambda} & & \text { (hypothesis) } \\
& \ll\left(X_{\imath}^{2-\lambda} X_{\jmath}^{2} X_{\imath+1}^{-4 \lambda}\right)^{\lambda} & & \text { (Lemma 2.3.1) } \\
& \ll X_{\imath+1}^{(2-\lambda)(1-\lambda)} X_{\jmath}^{2 \lambda} X_{\imath+1}^{-4 \lambda^{2}} & & \text { (Lemma 2.3.4) } \\
& \ll X_{\imath+1}^{(2-\lambda)(1-\lambda)}\left(X_{\imath+1} X_{l+1}\right)^{2 \lambda(1-\lambda)} X_{\imath+1}^{-4 \lambda^{2}} & & \text { (Corollary 4.3) } \\
& =X_{\imath+1}^{-\left(5 \lambda^{2}+\lambda-2\right)} X_{J+1}^{2 \lambda(1-\lambda)} & &
\end{aligned}
$$

Since $5 \lambda^{2}+\lambda-2$ and $2 \lambda(1-\lambda)$ are respectively increasing and decreasing functions of $\lambda$ for $\lambda>1 / 2$, the above estimate combined with our assumption that $\lambda>5 / 7$ leads to

$$
X_{\imath+1}^{62 / 49} \ll X_{j+1}^{20 / 49}
$$

and therefore

$$
X_{\imath+1}^{3} \leq X_{1+1}^{31 / 10} \ll X_{j+1}
$$

It then follows from Corollary 4.3 that

$$
X_{j}^{3} \ll X_{\jmath+1}^{4(1-\lambda)}
$$


By Corollary 7.5 , we also have $f\left(\mathbf{x}_{j}, \mathbf{x}_{k}\right)=0$ and Lemma 8.2 .3 now tells us that

$$
1 \ll X_{\jmath}^{4} X_{k} L_{\jmath} L_{k}+X_{\jmath}^{3} X_{\jmath+1} L_{\jmath}^{3}+X_{\jmath} X_{k}^{2} L_{\jmath}^{4}
$$

This means that at least one of the summands must be $\gg 1$. We now look at each summand separately and find that this is impossible.

We first suppose that $1 \ll X_{j}^{4} X_{k} L_{\jmath} L_{k}$. This gires

$$
\begin{aligned}
1 & \ll\left(X_{\jmath}^{4} X_{k} L_{\jmath} L_{k}\right)^{3(1-\lambda)} & & \text { (hypothesis) } \\
& \ll X_{\jmath}^{12(1-\lambda)} X_{k}^{-3(2 \lambda-1)} & & \text { (Corollary 4.3) } \\
& \ll X_{\jmath+1}^{16(1-\lambda)^{2}} X_{h}^{-3(2 \lambda-1)} & & \text { (equation }(8.3 .2) \text { ) }
\end{aligned}
$$

but we also have

$$
\begin{aligned}
1 & \ll\left(X_{\jmath} X_{k} L_{\jmath}^{2}\right)^{3} & & \text { (Corollary 5.5) } \\
& \ll\left(X_{\jmath} X_{k} X_{\jmath+1}^{-2 \lambda}\right)^{3} & & \text { (Lemma 2.3.1) } \\
& \ll X_{\jmath+1}^{-2(5 \lambda-2)} X_{h}^{3} & & \text { (equation (8.3.2)) } .
\end{aligned}
$$

Combining these two results we obtain

$$
1 \ll X_{J+1}^{-\left(2 \lambda^{2}+7 \lambda-6\right)}
$$

which is a contradiction for $y$ sufficiently large since $2 \lambda^{2}+7 \lambda-6>0$ when $\lambda>$ $(\sqrt{97}-7) / 4 \approx 0.712214$.

We now look at the second summand and suppose that $1 \ll X_{j}^{3} X_{J+1} L_{j}^{3}$. We then find:

$$
\begin{aligned}
1 & \ll X_{\jmath}^{3} X_{\jmath+1} L_{\jmath}^{3} & & \text { (hypothesis) } \\
& \ll X_{\jmath}^{3} X_{\jmath+1}^{1-3 \lambda} & & \text { (Lemma 2.3.1) } \\
& \ll X_{\jmath+1}^{-(7 \lambda-5)} & & \text { (equation (8.3 }
\end{aligned}
$$

which is a contradiction for $\jmath$ sufficiently large since $\lambda>5 / 7$. 
Therefore, we must have $1 \ll X_{j} X_{k}^{2} L_{j}^{4}$. However, this is in contradiction with Lemma 8.3.3.

Combining Corollary 7.5 and Lemma 8.3 .4 we can now suppose that if $\lambda>5 / 7$ is an exponent of approximation, then for all pairs of consecutive elements $i<j$ of $\mathbf{I}$ with $i$ sufficiently large, we have $f\left(\mathbf{x}_{i}, \mathbf{x}_{j}\right)=0$ and $g\left(\mathbf{x}_{i-1}, \mathbf{x}_{i}, \mathbf{x}_{j}\right)=0$. 


\section{Chapter 9}

\section{Finer estimates}

Let $\mathbf{T}=\left(1 . T . T^{3}\right)$ and $\underline{\underline{\xi}}=\left(1, \xi, \xi^{3}\right)$. As in the proof of Proposition 5.2, for a triple $\mathbf{x}=\left(x_{0}, x_{1}, x_{2}\right)$. we define

$$
\Delta \mathbf{x}=\mathbf{x}-x_{0} \underline{\underline{\xi}}=\left(0, \Delta x_{1}, \Delta x_{2}\right)
$$

We also define the polynomial

$$
P_{\mathbf{x}}(T):=\Phi(\mathbf{x}, \mathbf{T}, \mathbf{T})
$$

We then have

$$
P_{\mathbf{x}}(\xi)=\Phi(\mathbf{x}, \underline{\underline{\xi}}, \underline{\underline{\xi}})=\Phi\left(x_{0} \underline{\underline{\xi}}+\Delta \mathbf{x} . \underline{\underline{\xi}}, \underline{\underline{\xi}}\right)=\Phi(\Delta \mathbf{x}, \underline{\underline{\xi}}, \underline{\underline{\xi}})=P_{\Delta \mathbf{x}}(\xi)
$$

since $\Phi$ is multilinear and $\Phi(\mathbf{T}, \mathbf{T}, \mathbf{T})=0$. This gives the following upper bound:

$$
\left|P_{\mathbf{x}}(\xi)\right| \ll L(\mathbf{x})
$$

A short computation shows that

$$
P_{\mathbf{x}}(\xi)=\Delta x_{2}-3 \xi^{2} \Delta x_{1}
$$

and therefore

$$
L(\mathbf{x})=\max \left\{\left|\Delta x_{1}\right|,\left|\Delta x_{2}\right|\right\} \asymp \max \left\{\left|\Delta x_{1}\right|,\left|P_{\mathbf{x}}(\xi)\right|\right\}
$$


For that reason, and for simplicity, we replace the polynomial notation by the notation used in the proof of Proposition 5.2:

$$
\delta(\mathbf{x}):=\delta_{\xi}(\mathbf{x})=P_{\mathbf{x}}(\xi)
$$

and (9.0.2) then becomes

$$
L(\mathbf{x}) \asymp \max \left\{\left|\Delta x_{1}\right|,|\delta(\mathbf{x})|\right\} .
$$

In order to improve the upper bounds of the previous chapters, we will reexamine some results and refine them by taking expansions with respect to $\Delta x_{1}$ and $\delta(\mathbf{x})$ instead of $\Delta x_{1}$ and $\Delta x_{2}$.

\subsection{Finer estimates for $\varphi$}

For an arbitrary point $\mathbf{x} \in \mathbb{R}^{3}$, expanding $\varphi(\mathbf{x})$ as a polynomial in $x_{0}, \Delta x_{1}$ and $\delta(\mathbf{x})$, we find

$$
\varphi(\mathbf{x})=x_{0}^{2} \delta(\mathbf{x})+\mathcal{O}\left(\|\mathbf{x}\| L(\mathbf{x})^{2}\right)
$$

This leads to the following result which refines Lemma 3.10.

Lemma 9.1.1 Let $\xi \in \mathbb{R}$ be such that $1, \xi, \xi^{3}$ are linearly independent over $\mathbb{Q}$. Let $\left(\mathbf{x}_{i}\right)_{\imath \geq 1}$ be a sequence of minimal points associated to $\left(1, \xi, \xi^{3}\right)$. Suppose that $\lambda>2 / 3$ is an exponent of approximation to $\left(1, \xi, \xi^{3}\right)$. Then, for ench sufficiently large $i$, we have

$$
1 \leq\left|\varphi\left(\mathbf{x}_{i}\right)\right| \asymp X_{i}^{2}\left|\delta\left(\mathbf{x}_{i}\right)\right|
$$

Proof: The preceding estimate gives

$$
\varphi\left(\mathbf{x}_{i}\right)=x_{i, 0}^{2} \delta\left(\mathbf{x}_{i}\right)+\mathcal{O}\left(X_{i} L_{i}^{2}\right)
$$

Since $\lambda>2 / 3$, we have

$$
X_{1} L_{\imath}^{2} \ll X_{i}^{1-2 \lambda}<X_{,}^{-1 / 3}=o(1)
$$


The conclusion follows since by Lemma 3.9 we also have $\varphi\left(\mathbf{x}_{i}\right) \neq 0$ for $i$ sufficiently large which means that

$$
1 \leq\left|\varphi\left(\mathbf{x}_{i}\right)\right|
$$

The next lemma collects easy estimates that will be useful in the computations which follow.

Lemma 9.1.2 Let $\xi \in \mathbb{R}$ be such that $1, \xi . \xi^{3}$ are linearly independent over $\mathbb{Q}$. Let $\left(\mathbf{x}_{i}\right)_{\imath \geq 1}$ be a sequence of minimal points associated to $\left(1, \xi . \xi^{3}\right)$. Denote by $\mathbf{I}$ the set of all indices $i \geq 2$ for which $\mathbf{x}_{i-1}, \mathbf{x}_{i}, \mathbf{x}_{i+1}$ are linearly independent. Suppose that $\lambda>2 / 3$ is un exponent of approximation to $\left(1, \xi, \xi^{3}\right)$. Then for any pair of consecutive elements $i<j$ of $\mathbf{I}$ we have.

$$
\left|\delta\left(\mathbf{x}_{j}\right)\right| \ll\left|\delta\left(\mathbf{x}_{i}\right)\right|, \quad L_{\imath} L_{\jmath} \ll\left|\delta\left(\mathbf{x}_{\jmath}\right)\right| \quad \text { and } \quad L_{\imath} \ll\left|\delta\left(\mathbf{x}_{i}\right)\right| X_{i} .
$$

Proof: By Lemmas 2.3.6 and 9.1.1, we have

$$
\left|\delta\left(\mathbf{x}_{j}\right)\right| \ll L_{3} \ll X_{j}^{-4 / 3} \ll X_{\imath}^{-8 / 3} \ll\left|\delta\left(\mathbf{x}_{i}\right)\right|
$$

The second result follows from Corollary 4.3 and Lemma 9.1.1,

$$
L_{i} L_{j} \ll X_{j}^{-\lambda /(1-\lambda)} \leq X_{j}^{-2} \ll\left|\delta\left(\mathbf{x}_{j}\right)\right|
$$

Finally, by Lemmas 2.3.6 and 9.1.1. we have

$$
L_{i} \ll X_{i}^{-4 / 3} \leq Y_{i}^{-2} X_{i} \ll\left|\delta\left(\mathbf{x}_{i}\right)\right| X_{i}
$$




\subsection{Finer estimates for $g$}

We now turn our attention to $g$ and begin by establishing a general upper bound for $\left|g\left(\mathbf{x}, \mathbf{x}_{2}, \mathbf{x}_{j}\right)\right|$.

Lemma 9.2.1 Let $\xi \in \mathbb{R}$ be such that $1, \xi, \xi^{3}$ are linearly independent over $\mathbb{Q}$. Let $\left(\mathbf{x}_{i}\right)_{2 \geq 1}$ be a sequence of minimal points associated to $\left(1, \xi, \xi^{3}\right)$. Denote by $\mathbf{I}$ the set of all indices $i \geq 2$ for which $\mathbf{x}_{i-1} \cdot \mathbf{x}_{1}, \mathbf{x}_{i+1}$ are linearly independent. Suppose that $\lambda>2 / 3$ is an exponent of approximation to $\left(1, \xi, \xi^{3}\right)$. Then for any point $\mathbf{x}$ and any pair of consecutive elements $i<j$ of $\mathbf{I}$ we have

$$
\left|g\left(\mathbf{x}, \mathbf{x}_{i}, \mathbf{x}_{j}\right)\right| \ll\left|\delta ( \mathbf { x } _ { \imath } ) \left\|\delta\left(\mathbf{x}_{j}\right)\left|\|\mathbf{x}\| X_{i}^{2} X_{j}+\right| \delta(\mathbf{x})|| \delta\left(\mathbf{x}_{j}\right) \mid X_{\imath}^{-3} X_{j}+X_{j}^{2} L(\mathbf{x}) L_{i}^{3}\right.\right.
$$

Proof: Expanding $g(\mathbf{x}, \mathbf{y}, \mathbf{z})$ in terms of $x_{0}, \Delta x_{1}, \ldots, \delta(\mathbf{z})$, for arbitrary points $\mathbf{x}, \mathbf{y}, \mathbf{z} \in \mathbb{R}^{3}$, we find

$$
\begin{aligned}
g(\mathbf{x}, \mathbf{y}, \mathbf{z})= & -\delta(\mathbf{x}) \delta(\mathbf{z}) y_{0}^{3} z_{0}-3 \delta(\mathbf{y}) \delta(\mathbf{z}) x_{0} y_{0}^{2} z_{0}+\delta(\mathbf{z})^{2} x_{0} y_{0}^{3} \\
& +\mathcal{O}\left(\|\mathbf{z}\|^{2} L(\mathbf{x}) L(\mathbf{y})^{3}+\|\mathbf{y}\|^{2}\|\mathbf{z}\| L(\mathbf{x}) L(\mathbf{y}) L(\mathbf{z})+\|\mathbf{x}\|\|\mathbf{y}\|\|\mathbf{z}\| L(\mathbf{y})^{2} L(\mathbf{z})\right. \\
& \left.+\|\mathbf{y}\|^{3} L(\mathbf{x}) L(\mathbf{z})^{2}+\|\mathbf{x}\|\|\mathbf{y}\|^{2} L(\mathbf{y}) L(\mathbf{z})^{2}\right)
\end{aligned}
$$

In particular, for $i$ and $j$ as in the statement of the lemma, we get:

$$
\begin{aligned}
\left|g\left(\mathbf{x}, \mathbf{x}_{i}, \mathbf{x}_{j}\right)\right| \ll & \left|\delta(\mathbf{x})\left\|\delta\left(\mathbf{x}_{j}\right)\left|X_{i}^{3} X_{j}+\right| \delta\left(\mathbf{x}_{i}\right)\right\| \delta\left(\mathbf{x}_{j}\right)\right|\|\mathbf{x}\| X_{i}^{2} X_{j}+\left|\delta\left(\mathbf{x}_{j}\right)\right|^{2}\|\mathbf{x}\| X_{i}^{3} \\
& +X_{j}^{2} L(\mathbf{x}) L_{\imath}^{3}+X_{\imath}^{2} X_{j} L(\mathbf{x}) L_{i} L_{j}+\|\mathbf{x}\| X_{\imath} X_{j} L_{\imath}^{2} L_{j}
\end{aligned}
$$

The third term in this upper bound is superfluous because, by Lemma 9.1.2, we have:

$$
\left|\delta\left(\mathbf{x}_{\jmath}\right)\right|^{2}\|\mathbf{x}\| X_{i}^{3} \ll\left|\delta\left(\mathbf{x}_{i}\right)\left\|\delta\left(\mathbf{x}_{\jmath}\right) \mid\right\| \mathbf{x} \| X_{i}^{2} X_{j}\right.
$$

The fifth term can be dropped as well because, by Lemma 2.3.6, we have:

$$
X_{i}^{2} X_{J} L(\mathbf{x}) L_{i} L_{j} \ll X_{j}^{2} L(\mathbf{x}) L_{i}^{3}
$$


Finally, we observe that the last term can also be discarded because, using Lemma 9.1 .2 , we find

$$
\|\mathbf{x}\| X_{i} X_{j} L_{i}^{2} L_{\jmath} \ll\left|\delta\left(\mathbf{x}_{i}\right)\left\|\delta\left(\mathbf{x}_{j}\right) \mid\right\| \mathbf{x} \| X_{i}^{2} X_{\jmath}\right.
$$

Corollary 9.2.2 Let $\xi \in \mathbb{R}$ be such that $1, \xi, \xi^{3}$ are linearly independent over $\mathbb{Q}$. Let $\left(\mathbf{x}_{i}\right)_{2 \geq 1}$ be a sequcnce of minimal points associated to $\left(1, \xi, \xi^{3}\right)$. Denote by $\mathbf{I}$ the set of all indices $i \geq 2$ for which $\mathbf{x}_{\imath-1}, \mathbf{x}_{1}, \mathbf{x}_{1+1}$ are linearly independent. Suppose that $\lambda>2 / 3$ is an exponent of approximation to $\left(1 . \xi, \xi^{3}\right)$. Then for any pair of consecutive elements $i<j$ of $\mathbf{I}$ with $f\left(\mathbf{x}_{i}, \mathbf{x}_{\jmath}\right)=0$ we have

$$
1 \ll\left|\delta\left(\mathbf{x}_{\imath}\right)\right|\left|\delta\left(\mathbf{x}_{j}\right)\right| X_{\imath}^{2} X_{\imath+1} X_{j}+X_{j}^{2} L_{i}^{4}
$$

Proof: $\quad$ Since $f\left(\mathbf{x}_{i}, \mathbf{x}_{\jmath}\right)=0$. by Proposition 7.2 we have $g\left(\mathbf{x}_{i+1}, \mathbf{x}_{i}, \mathbf{x}_{\jmath}\right) \neq 0$. By Lemma 9.2.1, we then get:

$$
\begin{aligned}
1 & \ll\left|\delta\left(\mathbf{x}_{\imath}\right)\right|\left|\delta\left(\mathbf{x}_{j}\right)\right| X_{i}^{2} X_{i+1} X_{j}+\left|\delta\left(\mathbf{x}_{i+1}\right)\right|\left|\delta\left(\mathbf{x}_{j}\right)\right| X_{i}^{3} X_{j}+X_{j}^{2} L_{i}^{3} L_{i+1} \\
& \ll\left|\delta\left(\mathbf{x}_{i}\right)\right|\left|\delta\left(\mathbf{x}_{\jmath}\right)\right| X_{i}^{2} X_{i+1} X_{j}+\left|\delta\left(\mathbf{x}_{j}\right)\right| X_{i}^{3} X_{j} L_{i}+X_{j}^{2} L_{i}^{4}
\end{aligned}
$$

In the latter estimate, the second term can be dropped because using first Lemma 9.1.2 and then Lemma 2.3.6 we find

$$
X_{\imath} L_{i} \ll X_{i}^{2}\left|\delta\left(\mathbf{x}_{i}\right)\right| \ll X_{\imath+1}\left|\delta\left(\mathbf{x}_{i}\right)\right|
$$

which implies that $\left|\delta\left(\mathbf{x}_{j}\right)\right| X_{i}^{3} X_{J} L_{i} \ll\left|\delta\left(\mathbf{x}_{i}\right)\right|\left|\delta\left(\mathbf{x}_{j}\right)\right| X_{i}^{2} X_{i+1} X_{j}$.

This leads to the following refinement of Corollary 7.7 .

Corollary 9.2.3 Let $\xi \in \mathbb{R}$ be such that $1, \xi . \xi^{3}$ are linearly indcpendent over $\mathbb{Q}$. Let $\left(\mathbf{x}_{i}\right)_{\imath \geq 1}$ be a sequence of minimal points associated to $\left(1, \xi, \xi^{3}\right)$. Denote by $\mathbf{I}$ the 
set of all indices $i \geq 2$ for which $\mathbf{x}_{-1}, \mathbf{x}_{1}, \mathbf{x}_{i+1}$ are linearly independent. Suppose that $\lambda>5 / 7$ is an exponent of approximation to $\left(1, \xi, \xi^{3}\right)$. Then for any pair of consecutive elements $i<j$ of $\mathbf{I}$ we have

$$
1 \ll\left|\delta\left(\mathbf{x}_{i}\right)\right|\left|\delta\left(\mathbf{x}_{j}\right)\right| X_{1}^{2} X_{i+1} X_{\jmath}
$$

Proof: $\quad$ Since $\lambda>5 / 7$ we have $f\left(\mathbf{x}_{i}, \mathbf{x}_{j}\right)=0$ by Corollary 7.5. From Corollary 9.2 .2 we then get

$$
1 \ll\left|\delta\left(\mathbf{x}_{i}\right)\right|\left|\delta\left(\mathbf{x}_{j}\right)\right| X_{\imath}^{2} X_{i+1} X_{j}+X_{j}^{2} L_{i}^{4}
$$

The conclusion follows since by Lemma 7.3, we have $X_{j}^{2} L_{i}^{4}=o(1)$.

\subsection{A further consequence of the vanishing of $f$}

In the next lemma, we follow essentially the same steps as in the proof of Lemma 9.2.1 to expand $f\left(\mathbf{x}_{i}, \mathbf{x}_{j}\right)=g\left(\mathbf{x}_{i}, \mathbf{x}_{i}, \mathbf{x}_{\jmath}\right)$. When $f\left(\mathbf{x}_{i}, \mathbf{x}_{j}\right)=0$ the main term of this expansion is bounded above in absolute value by the sum of the other terms. This gives:

Lemma 9.3.1 Let $\xi \in \mathbb{R}$ be such that $1 . \xi, \xi^{3}$ are linearly independent over $\mathbb{Q}$. Let $\left(\mathbf{x}_{i}\right)_{\imath \geq 1}$ be a sequence of minimal points associated to $\left(1, \xi, \xi^{3}\right)$. Denote by $\mathbf{I}$ the set of all indices $i \geq 2$ for which $\mathbf{x}_{i-1}, \mathbf{x}_{i}, \mathbf{x}_{i+1}$ are linearly independent. Suppose that $\lambda>5 / 7$ is an exponent of approxumation to $\left(1, \xi, \xi^{3}\right)$. Then for any pair of consecutive elements $i<j$ of $\mathbf{I}$ we have

$$
\left|\delta\left(\mathbf{x}_{r}\right)\right|\left|\delta\left(\mathbf{x}_{j}\right)\right| X_{i}^{3} \ll X_{j} L_{i}^{4}
$$


Proof: Expanding $f(\mathbf{y}, \mathbf{z})$ in terms of $y_{0}, \Delta y_{1}, \ldots, \delta(\mathbf{z})$, for arbitrary points $\mathbf{x}, \mathbf{y}, \mathbf{z} \in$ $\mathbb{R}^{3}$, we find

$$
\begin{aligned}
f(\mathbf{y}, \mathbf{z})=g(\mathbf{y}, \mathbf{y}, \mathbf{z})= & -4 \delta(\mathbf{y}) \delta(\mathbf{z}) y_{0}^{3} z_{0}+\delta(\mathbf{z})^{2} y_{0}^{4} \\
& +\mathcal{O}\left(\|\mathbf{z}\|^{2} L(\mathbf{y})^{4}+\|\mathbf{y}\|^{2}\|\mathbf{z}\| L(\mathbf{y})^{2} L(\mathbf{z})+\|\mathbf{y}\|^{3} L(\mathbf{y}) L(\mathbf{z})^{2}\right)
\end{aligned}
$$

In particular for $i$ and $j$ as in the statement of the theorem, we have $f\left(\mathbf{x}_{i}, \mathbf{x}_{j}\right)=0$. This now gives

$$
\left|\delta\left(\mathbf{x}_{i}\right)\right|\left|\delta\left(\mathbf{x}_{j}\right)\right| X_{i}^{3} X_{j} \ll\left|\delta\left(\mathbf{x}_{j}\right)\right|^{2} X_{i}^{4}+X_{j}^{2} L_{i}^{4}+X_{i}^{2} X_{j} L_{i}^{2} L_{j}
$$

The conclusion follows because, by Lemma 2.3.6, we have

$$
\left|\delta\left(\mathbf{x}_{j}\right)\right|^{2} X_{i}^{4} \ll X_{i}^{4} L_{j}^{2} \ll X_{i}^{2} X_{j} L_{i}^{2} L_{j} \ll X_{j}^{2} L_{i}^{4}
$$

\subsection{Finer estimates for $S$}

Expanding $S(\mathbf{x}, \mathbf{y}, \mathbf{z})$ in terms of $x_{0}, \Delta x_{1}, \ldots, \delta(\mathbf{z})$ we obtain:

$$
\begin{aligned}
S(\mathbf{x}, \mathbf{y}, \mathbf{z})= & \left(\delta(\mathbf{y}) \delta(\mathbf{z}) x_{0}^{5}-4 \delta(\mathbf{x}) \delta(\mathbf{z}) x_{0}^{4} y_{0}\right) \underline{\underline{\xi}} \\
+ & \mathcal{O}\left(\|\mathbf{x}\|\|\mathbf{y}\|\|\mathbf{z}\| L(\mathbf{x})^{4}+\|\mathbf{x}\|^{3}\|\mathbf{y}\| L(\mathbf{x})^{2} L(\mathbf{z})\right. \\
& \left.+\|\mathbf{x}\|^{3}\|\mathbf{z}\| L(\mathbf{x})^{2} L(\mathbf{y})+\|\mathbf{x}\|^{4} L(\mathbf{x}) L(\mathbf{y}) L(\mathbf{z})\right)
\end{aligned}
$$

Lemma 9.4.1 Let $\xi \in \mathbb{R}$ be such that $1, \xi, \xi^{3}$ are linearly independent over $\mathbb{Q}$. Let $\left(\mathbf{x}_{i}\right)_{i \geq 1}$ be a sequence of minimal points associated to $\left(1, \xi, \xi^{3}\right)$. Denote by $\mathbf{I}$ the set of all indices $i \geq 2$ for which $\mathbf{x}_{i-1}, \mathbf{x}_{i}, \mathbf{x}_{i+1}$ are linearly independent. Suppose that $\lambda>5 / 7$ is an exponent of approximation to $\left(1, \xi, \xi^{3}\right)$. Then for any $i \in \mathbf{I}$ we have

$$
1 \ll X_{i}^{3} X_{i+1} L_{i}^{3}
$$


Proof: Let $\imath<j$ be a pair of consecutive elements of $\mathbf{I}$ with 7 arbitrarily large. By Corollary 7.5 we have $f\left(\mathbf{x}_{1}, \mathbf{x}_{\jmath}\right)=0$.

We first note that by Proposition 8.2 .2 we have $S\left(\mathbf{x}_{2}, \mathbf{x}_{1-1}, \mathbf{x}_{\jmath}\right) \neq 0$ since $\mathbf{x}_{\jmath-1}$ is a linear combination of $x_{1}$ and $x_{j}$, but not a multiple of $\mathbf{x}_{j}$. Using the expansion of $S$ given above. we then find

$$
\begin{aligned}
1 & \leq\left\|S\left(\mathbf{x}_{\imath}, \mathbf{x}_{\jmath-1} \cdot \mathbf{x}_{\jmath}\right)\right\| \\
& \ll\left|\delta\left(\mathbf{x}_{\jmath-1}\right)\right|\left|\delta\left(\mathbf{x}_{\jmath}\right)\right| X_{\imath}^{5}+\left|\delta\left(\mathbf{x}_{\imath}\right)\right|\left|\delta\left(\mathbf{x}_{\jmath}\right)\right| X_{\imath}^{4} X_{\jmath-1}+X_{\imath} X_{3-1} X_{\jmath} L_{\imath}^{4}+X_{\imath}^{3} X_{\jmath} L_{\imath}^{2} L_{\jmath-1} \\
& \ll\left|\delta\left(\mathbf{x}_{\jmath}\right)\right| X_{\imath}^{\jmath} L_{\jmath-1}+\left|\delta\left(\mathbf{x}_{\imath}\right)\right|\left|\delta\left(\mathbf{x}_{\jmath}\right)\right| X_{\imath}^{4} X_{\jmath}+X_{\imath} X_{\jmath}^{2} L_{\imath}^{4}+X_{\imath}^{3} X_{\jmath} L_{\imath}^{2} L_{\jmath-1} \\
& \simeq\left|\delta\left(\mathbf{x}_{\jmath}\right)\right| X_{\imath}^{5} L_{\jmath-1}+\left|\delta\left(\mathbf{x}_{\imath}\right)\right|\left|\delta\left(\mathbf{x}_{\jmath}\right)\right| X_{\imath}^{4} X_{\jmath}+X_{\imath} X_{\jmath}^{2} L_{\imath}^{4}+X_{\imath}^{3} X_{\imath+1} L_{\imath}^{3}
\end{aligned}
$$

where the last step is obtained by applying Corollary 4.3 to eliminate $L_{\jmath-1}$ from the last term in the RHS. We now show that the first and the second term in the RHS of this equation are superfluous. The first one is superfluous because

$$
\begin{aligned}
X_{\imath} L_{\jmath}-1 & \leq X_{\imath} L_{\imath} & & \left(L_{\jmath-1} \leq L_{\imath}\right) \\
& \ll X_{\imath}^{2}\left|\delta\left(\mathbf{x}_{\imath}\right)\right| & & (\text { Lemma } 9.1 .2) \\
& \ll X_{\imath+1}\left|\delta\left(\mathbf{x}_{\imath}\right)\right| & & (\text { Lemma 2.3.6) } \\
& \leq X_{\jmath}\left|\delta\left(\mathbf{x}_{\imath}\right)\right| & & \left(X_{\imath+1} \leq X_{\jmath}\right)
\end{aligned}
$$

and so we find that $\left|\delta\left(\mathbf{x}_{\jmath}\right)\right| X_{\imath}^{5} L_{\jmath-1} \ll\left|\delta\left(\mathbf{x}_{\imath}\right)\right|\left|\delta\left(\mathbf{x}_{j}\right)\right| X_{\imath}^{4} X_{\jmath}$. For the second term, Lemma 9.3.1 immediately gives us that $\left|\delta\left(\mathbf{x}_{2}\right)\right|\left|\delta\left(\mathbf{x}_{3}\right)\right| X_{1}^{4} X_{3} \ll X_{1} X_{3}^{2} L_{\imath}^{4}$. We are now left with

$$
1 \ll X_{1} X_{3}^{2} L_{\imath}^{4}+X_{2}^{3} X_{\imath+1} L_{7}^{3}
$$

The conclusion follows since by Lemma 8.3.3, we have $X_{1} X_{j}^{2} L_{\imath}^{4}=o(1)$.

Theorem 9.4.2 Let $\xi \in \mathbb{R}$ be such that $1 . \xi, \xi^{3}$ are lancarly independent over $\mathbb{Q}$. If $\lambda$ is an exponent of approximation to $\left(1, \xi, \xi^{3}\right)$, then $\lambda \leq(9+\sqrt{15}) / 18 \approx 0.7151657$ 
Proof: Suppose by contradiction that $\lambda>(9+\sqrt{15}) / 18$. Let $\left(\mathbf{x}_{\imath}\right)_{\imath \geq 1}$ be a sequence of minimal points associated to $\left(1, \xi, \xi^{3}\right)$. Denote by $\mathbf{I}$ the set of all indices $i \geq 2$ for which $\mathbf{x}_{\imath-1}, \mathbf{x}_{\imath}, \mathbf{x}_{\imath+1}$ are linearly independent. Let $i<j$ be a pair of consecutive elements of $\mathbf{I}$ with $\imath$ arbitrarily large.

Combining Corollary 9.2.3 and Lemma 9.3.1 we have

$$
X_{\imath} \ll X_{\imath+1} X_{\jmath}^{2} L_{\imath}^{4}
$$

We then find that:

$$
\begin{aligned}
1 & \ll X_{\imath}^{3} X_{\imath+1} L_{\imath}^{3} & & \text { (Lemma 9.4.1) } \\
& \ll X_{\imath+1}^{4} X_{\jmath}^{6} L_{\imath}^{15} & & \text { (equation (9.4.1)) } \\
& \ll X_{\imath+1}^{4-15 \lambda} X_{\jmath}^{6} & & \text { (Lemma 2.3.1) } \\
& \ll X_{\imath+1}^{4-15 \lambda}\left(X_{\imath+1} X_{\jmath+1}\right)^{6(1-\lambda)} & & \text { (Corollary 4.3) } \\
& =X_{\imath+1}^{10-21 \lambda} X_{\jmath+1}^{6(1-\lambda)} . & &
\end{aligned}
$$

But we also have:

$$
\begin{aligned}
1 & \ll X_{j}^{3} X_{j+1} L_{j}^{3} \\
& \ll X_{j}^{3} X_{j+1}^{1-3 \lambda} \\
& \ll\left(X_{\imath+1} X_{J+1}\right)^{3(1-\lambda)} X_{\jmath+1}^{1-3 \lambda} \\
& =X_{\imath+1}^{3(1-\lambda)} X_{J+1}^{2(2-3 \lambda)} .
\end{aligned}
$$

Combining these two results we obtain

$$
1 \ll X_{\imath+1}^{-2\left(54 \lambda^{2}-54 \lambda+11\right)}
$$

which would lead to a contradiction if $\lambda>(9+\sqrt{15}) / 18$ and $i$ is sufficiently large.

We end this chapter by noting that the upper bound provided by this theorem is not optimal. It can be slightly improved using the fact that $g\left(\mathbf{x}_{\imath-1}, \mathbf{x}_{i}, \mathbf{x}_{j}\right)=0$ 
when $\lambda>5 / 7$. In order to do this, one needs an analog of Lemma 9.3.1 where instead of decomposing $f\left(\mathbf{x}_{i}, \mathbf{x}_{j}\right)$, we decompose $g\left(\mathbf{x}_{i-1}, \mathbf{x}_{i}, \mathbf{x}_{j}\right)$ and observe that, since $g\left(\mathbf{x}_{i-1}, \mathbf{x}_{\imath}, \mathbf{x}_{j}\right)=0$, the "main term" is bounded above in absolute value by the sum of the other terms. 


\section{Chapter 10}

\section{The function $k$ and the last}

\section{estimate}

In this chapter we come to the main result of this thesis by showing that $5 / 7$ is an upper bound for any exponent of simultaneous approximation to $\left(1, \xi, \xi^{3}\right)$, when $1, \xi . \xi^{3}$ are linearly independent over $\mathbb{Q}$. This result relies on properties of the following function.

Definition 10.1 We set

$$
k(\mathbf{x}, \mathbf{y}, \mathbf{z})=g(\psi(\mathbf{x}, \mathbf{z}), \mathbf{x}, \mathbf{y})+\varphi(\mathbf{x})^{2} \Phi(\mathbf{y}, \mathbf{y}, \mathbf{z}) .
$$

Note that $k$ satisfies the following remarkable property:

Proposition 10.2

$$
k(\mathbf{x}, a \mathbf{x}+b \mathbf{y}, \mathbf{y})=b^{2}\left(\Phi(\mathbf{x}, \mathbf{x} \cdot \mathbf{y}) f(\mathbf{x}, \mathbf{y})+9 \varphi(\mathbf{x})^{2} \varphi(\mathbf{y})\right)
$$

As an immediate consequence we get the following corollary.

Corollary 10.3 Let $\xi \in \mathbb{R}$ be such that $1, \xi, \xi^{3}$ are linearly independent over $\mathbb{Q}$. Let $\left(\mathbf{x}_{i}\right)_{i \geq 1}$ be a sequence of minimal points associated to $\left(1, \xi, \xi^{3}\right)$. Denote by $\mathbf{I}$ the set 
of all indices $i \geq 2$ for which $\mathbf{x}_{i-1}, \mathbf{x}_{i}, \mathbf{x}_{i+1}$ are linearly independent. Suppose that $\lambda>2 / 3$ is an exponent of approximation to $\left(1, \xi, \xi^{3}\right)$. Let $i<j$ be a pair consecutive elcments of $\mathbf{I}$ and let $\mathbf{x}=a \mathbf{x}_{\imath}+b \mathbf{x}_{j}$ for some constants $a, b$ with $b \neq 0$. Then, if $i$ is sufficiently large we have $f\left(\mathbf{x}_{i}, \mathbf{x}_{\jmath}\right) \neq 0$ or $k\left(\mathbf{x}_{1}, \mathbf{x} . \mathbf{x}_{\jmath}\right) \neq 0$. In particular,

$$
\text { if } f\left(\mathbf{x}_{i}, \mathbf{x}_{j}\right)=0 \text { then } k\left(\mathbf{x}_{i}, \mathbf{x}_{i+1}, \mathbf{x}_{j}\right) \neq 0 \text {. }
$$

Proof: $\quad$ Suppose $f\left(\mathbf{x}_{\imath}, \mathbf{x}_{\jmath}\right)=0$. Then, by Proposition 10.2 we find:

$$
\begin{aligned}
k\left(\mathbf{x}_{i}, \mathbf{x}, \mathbf{x}_{\jmath}\right)=k\left(\mathbf{x}_{i}, a \mathbf{x}_{\imath}+b \mathbf{x}_{j}, \mathbf{x}_{j}\right) & =b^{2}\left(\Phi\left(\mathbf{x}_{i}, \mathbf{x}_{i}, \mathbf{x}_{\jmath}\right) f\left(\mathbf{x}_{i}, \mathbf{x}_{\jmath}\right)+9 \varphi\left(\mathbf{x}_{\imath}\right)^{2} \varphi\left(\mathbf{x}_{\jmath}\right)\right) \\
& =9 b^{2} \varphi\left(\mathbf{x}_{i}\right)^{2} \varphi\left(\mathbf{x}_{\jmath}\right) .
\end{aligned}
$$

By Lemma 3.9 this gives $k\left(\mathbf{x}_{i}, \mathbf{x}, \mathbf{x}_{j}\right) \neq 0$ if $i$ is sufficiently large.

The second assertion follows since $\mathbf{x}_{2+1}$ is a linear combination of $\mathbf{x}_{\imath}$ and $\mathbf{x}_{j}$ but not a multiple of $\mathbf{x}_{1}$.

Combining this result with estimates for $k\left(\mathbf{x}_{i}, \mathbf{x}_{i+1}, \mathbf{x}_{j}\right)$, we get:

Lemma 10.4 Let $\xi \in \mathbb{R}$ be such that $1, \xi, \xi^{3}$ are linearly independent over $\mathbb{Q}$. Let $\left(\mathbf{x}_{i}\right)_{1 \geq 1}$ be a sequence of minimal points associated to $\left(1, \xi, \xi^{3}\right)$. Denote by $\mathbf{I}$ the set of all indices $i \geq 2$ for which $\mathbf{x}_{-1}, \mathbf{x}_{i} . \mathbf{x}_{\imath+1}$ are linearly independent. Suppose that $\lambda>2 / 3$ is an exponent of approximation to $\left(1, \xi, \xi^{3}\right)$. Then for any pair of consecutive elements $i<j$ of $\mathbf{I}$ with $f\left(\mathbf{x}_{i}, \mathbf{x}_{j}\right)=0$ we have

$$
1 \ll\left|\delta\left(\mathbf{x}_{i}\right)\right|\left|\delta\left(\mathbf{x}_{j}\right)\right| X_{i}^{4} X_{i+1}^{2} L_{i}+X_{i} X_{i+1}^{2} X_{\jmath} L_{i}^{5}+X_{\imath}^{5} X_{i+1} L_{i}^{2} L_{\jmath}+X_{i}^{4} X_{j} L_{i}^{4} .
$$

Proof: Upon expanding $k(\mathbf{x}, \mathbf{y}, \mathbf{z})$ in terms of $x_{0} . \Delta x_{1}, \ldots, \delta(\mathbf{z})$, for arbitrary points $\mathbf{x}, \mathbf{y}, \mathbf{z} \in \mathbb{R}^{3}$, we get:

$$
\begin{aligned}
k(\mathbf{x}, \mathbf{y} \cdot \mathbf{z})= & \delta(\mathbf{x})^{2} \delta(\mathbf{z}) x_{0}^{4} y_{0}^{2} \\
+ & \mathcal{O}\left(\|\mathbf{x}\|\|\mathbf{y}\|^{2}\|\mathbf{z}\| L(\mathbf{x})^{5}+\|\mathbf{x}\|^{2}\|\mathbf{y}\|\|\mathbf{z}\| L(\mathbf{x})^{4} L(\mathbf{y})+\|\mathbf{x}\|^{3}\|\mathbf{y}\|^{2} L(\mathbf{x})^{3} L(\mathbf{z})\right. \\
& \left.+\|\mathbf{x}\|^{4}\|\mathbf{z}\| L(\mathbf{x})^{2} L(\mathbf{y})^{2}+\|\mathbf{x}\|^{5}\|\mathbf{y}\| L(\mathbf{x}) L(\mathbf{y}) L(\mathbf{z})+\|\mathbf{x}\|^{6} L(\mathbf{y})^{2} L(\mathbf{z})\right)
\end{aligned}
$$


If $\mathbf{x}, \mathbf{y}, \mathbf{z}$ are minimal points with $\|\mathbf{x}\| \leq\|\mathbf{y}\| \leq\|\mathbf{z}\|$ then we have $L(\mathbf{x}) \geq L(\mathbf{y}) \geq L(\mathbf{z})$. So. the second summand in the error term is bounded above by the first, while the sixth summand is bounded above by the fifth, and the estimate becomes

$$
\begin{aligned}
k(\mathbf{x}, \mathbf{y} \cdot \mathbf{z})=\delta(\mathbf{x})^{2} \delta(\mathbf{z}) x_{0}^{4} y_{0}^{2}+ & \mathcal{O}\left(\|\mathbf{x}\|\|\mathbf{y}\|^{2}\|\mathbf{z}\| L(\mathbf{x})^{j}+\|\mathbf{x}\|^{3}\|\mathbf{y}\|^{2} L(\mathbf{x})^{3} L(\mathbf{z})\right. \\
& \left.+\|\mathbf{x}\|^{4}\|\mathbf{z}\| L(\mathbf{x})^{2} L(\mathbf{y})^{2}+\|\mathbf{x}\|^{5}\|\mathbf{y}\| L(\mathbf{x}) L(\mathbf{y}) L(\mathbf{z})\right)
\end{aligned}
$$

In particular, for $\imath$ and $J$ as in the statement of the lemma, we get

$$
\begin{aligned}
\left|k\left(\mathbf{x}_{\imath}, \mathbf{x}_{\imath+1} \cdot \mathbf{x}_{l}\right)\right| \ll & \left|\delta\left(\mathbf{x}_{\imath}\right)\right|\left|\delta\left(\mathbf{x}_{\imath}\right)\right| X_{\imath}^{4} X_{\imath+1}^{2} L_{\imath}+X_{\imath} X_{\imath+1}^{2} X_{\jmath} L_{\imath}^{5} \\
& +X_{\imath}^{3} X_{\imath+1}^{2} L_{\imath}^{3} L_{J}+X_{\imath}^{4} X_{\jmath} L_{\imath}^{4}+X_{\imath}^{5} X_{\imath+1} L_{\imath}^{2} L_{\jmath}
\end{aligned}
$$

In this estimate, the third term is superfluous since Lemma 2.3 .6 gives

$$
X_{\imath}^{3} X_{1+1}^{2} L_{\imath}^{3} L_{\jmath} \ll X_{\imath} X_{\imath+1}^{2} X_{\jmath} L_{\imath}^{5}
$$

The conclusion follows since Corollary 10.3 shows that $k\left(\mathbf{x}_{\iota}, \mathbf{x}_{\imath+1}, \mathbf{x}_{J}\right)$ is a non-zero integer when $l$ is sufficiently large.

We can now prove our main result.

Theorem 10.5 Let $\xi \in \mathbb{R}$ be such that $1, \xi, \xi^{3}$ are linearly independent over $\mathbb{Q}$. If $\lambda$ is an exponcnt of approximation to $\left(1 . \xi . \xi^{3}\right)$, then $\lambda \leq 5 / 7$.

Proof: Suppose by contradiction that $\lambda>5 / 7$. Let $\left(\mathbf{x}_{i}\right)_{i \geq 1}$ be a sequence of minimal points associated to $\left(1, \xi, \xi^{3}\right)$. Denote by $\mathbf{I}$ the set of all indices $\iota \geq 2$ for which $\mathbf{x}_{\imath-1}, \mathbf{x}_{\imath}, \mathbf{x}_{\imath+1}$ are linearly independent. Let $\imath<\jmath<k$ be a triple of consecutive elements of $\mathbf{I}$ with $\imath$ arbitıarily large. By Corollary 7.5 we have that $f\left(\mathbf{x}_{\imath}, \mathbf{x}_{\mathfrak{\jmath}}\right)=$ $f\left(\mathbf{x}_{j}, \mathbf{x}_{k}\right)=0$. Then. Lemma 10.4 provides

$$
1 \ll\left|\delta\left(\mathbf{x}_{\jmath}\right)\right|\left|\delta\left(\mathbf{x}_{k}\right)\right| X_{\jmath}^{4} X_{\jmath+1}^{2} L_{\jmath}+X_{l} X_{\jmath+1}^{2} X_{k} L_{\jmath}^{5}+X_{\jmath}^{5} X_{\jmath+1} L_{\jmath}^{2} L_{k}+X_{\jmath}^{4} X_{k} L_{\jmath}^{4}
$$


We note that the last term in the RHS is superfluous siner it is related to the serond term by the inequality $X_{j}^{4} X_{h} L_{j}^{4} \ll X_{J} X_{j+1}^{2} X_{h} L_{j}^{5}$. This follows fiom:

$$
\begin{aligned}
X_{1}^{-3} & \ll X_{j}^{5} L_{\jmath} & & \text { (Lemma 3.10) } \\
& \ll X_{\jmath+1}^{5(1-\lambda) / \lambda} L_{\jmath} & & (\text { Lemma 2.3.4) } \\
& \leq X_{3+1}^{2} L_{\jmath} & & (\lambda>5 / \overline{7}) .
\end{aligned}
$$

Similarly. the third and the first tcrms satisfy $X_{j}^{5} X_{j+1} L_{j}^{2} L_{k} \ll X_{j}^{4} X_{\jmath+1}^{2} L_{\jmath}\left|\delta\left(\mathbf{x}_{\jmath}\right)\right|\left|\delta\left(\mathbf{x}_{k}\right)\right|$ because

$$
\begin{aligned}
X_{\jmath} L_{\jmath} L_{k} & \ll X_{\jmath} X_{k}^{-\lambda /(1-\lambda)} & & \text { (Corollary 4.3) } \\
& \ll X_{j}^{3} X_{k}^{-(3 \lambda-2) /(1-\lambda)}\left|\delta\left(\mathbf{x}_{\jmath}\right)\right|\left|\delta\left(\mathbf{x}_{k}\right)\right| & & \text { (Lemma 9.1. } \\
& \ll X_{j+1}^{3 / 2} X_{k}^{-(3 \lambda-2) /(1-\lambda)}\left|\delta\left(\mathbf{x}_{\jmath}\right)\right|\left|\delta\left(\mathbf{x}_{k}\right)\right| & & \text { (Lemma 2.3.6) } \\
& \ll X_{\jmath+1}^{-1-(7 \lambda-5) /(2-2 \lambda)}\left|\delta\left(\mathbf{x}_{\jmath}\right) \| \delta\left(\mathbf{x}_{k}\right)\right| & & \left(X_{J+1} \leq X_{h}\right) \\
& \leq X_{3+1}\left|\delta\left(\mathbf{x}_{\jmath}\right) \| \delta\left(\mathbf{x}_{k}\right)\right| & & (\lambda>5 / 7) .
\end{aligned}
$$

We also note that the first term is also superfluous since by Lemma 9.3 .1 we have:

$$
\left|\delta\left(\mathbf{x}_{\jmath}\right)\right|\left|\delta\left(\mathbf{x}_{h}\right)\right| X_{\jmath}^{4} X_{\jmath+1}^{2} L_{\jmath} \ll X_{\jmath} X_{\jmath+1}^{2} X_{k} L_{\jmath}^{5} .
$$

Therefore. we must have $1 \ll X_{\jmath} X_{j+1}^{2} X_{k} L_{j}^{5}$. In order to analyse this fact, we first show that

$$
X_{j}^{-2(7 \lambda-4)} \ll X_{j+1}^{(1-\lambda)(8 \lambda-3)}
$$

Indeed, we have

$$
\begin{aligned}
1 & \ll\left(X_{\imath}^{2} X_{\imath+1} X_{\jmath}\left|\delta\left(\mathbf{x}_{2}\right) \| \delta\left(\mathbf{x}_{l}\right)\right|\right)^{3} & & \text { (Corollary 9.2.3) } \\
& \ll X_{\imath+1}^{3} X_{\jmath}^{j} L_{\imath}^{8}\left|\delta\left(\mathbf{x}_{\imath}\right) \| \delta\left(\mathbf{x}_{\jmath}\right)\right| & & \text { (Lemma 9.3.1) } \\
& \ll X_{\imath+1}^{-(8 \lambda-3)} X_{\jmath}^{5} L_{\imath} L_{\jmath} & & \text { (equation }(9.0 .1)) \\
& \ll\left(X_{\jmath}^{-1 /(1-\lambda)} X_{\jmath+1}\right)^{8 \lambda-3} X_{\jmath}^{5-\lambda /(1-\lambda)} & & \text { (Corollary 4.3) } \\
& =X_{\jmath}^{-2(7 \lambda-4) /(1-\lambda)} X_{\jmath-1}^{8 \lambda-3} . & &
\end{aligned}
$$


This now gives:

$$
\begin{aligned}
1 & \ll\left(X_{\jmath} X_{j+1}^{2} X_{k} L_{j}^{5}\right)^{2(7 \lambda-4)} & & \text { (hypothesis) } \\
& \ll X_{j+1}^{(1-\lambda)(8 \lambda-3)+4(7 \lambda-4)}\left(X_{k} L_{j}^{5}\right)^{2(7 \lambda-4)} & & \text { (equation }(10.1)) \\
& \ll X_{\jmath+1}^{(1-\lambda)(8 \lambda-3)-2(5 \lambda-2)(7 \lambda-4)} X_{k}^{2(7 \lambda-4)} & & \text { (Lemma 2.3.1) }
\end{aligned}
$$

But we also have:

$$
\begin{aligned}
1 & \ll X_{j}^{2} X_{j+1} X_{k} L_{j} L_{k} & & \text { (Corollary 9.2.3) } \\
& \ll X_{j}^{2} X_{j+1} X_{k}^{1-\lambda /(1-\lambda)} & & \text { (Corollary 4.3) } \\
& \ll X_{j+1}^{(1-\lambda)(8 \lambda-3) /(7 \lambda-4)+1} X_{k}^{1-\lambda /(1-\lambda)} & & \text { (equation (10.1)) }
\end{aligned}
$$

Combining the two results we find

$$
1 \ll X_{J+1}^{-\frac{(2 \lambda-1)(10 \lambda-1)(7 \lambda-5)}{2(7 \lambda-4)(1-\lambda)}}
$$

which is a contradiction for $\lambda>5 / 7$ and $j$ sufficiently large. 


\section{Appendix A}

\section{Finding new relations}

The result presented in this appendix is the main tool that I used to find the various points and polynomials that appear throughout this thesis. It provides sufficient conditions for a polynomial to vanish when evaluated at a number of consecutive elements of a sequence of minimal points associated to a triple of the form $\left(1, \xi, \xi^{3}\right)$. The argument is adapted from Appendix $\mathrm{A}$ in [9] which deals with the case of $\left(1, \xi, \xi^{2}\right)$.

Let $\xi \in \mathbb{R}, \sigma_{1}=1 . \sigma_{2}=3$ and let $\left(\mathbf{x}_{i}\right)_{i \geq 1}$ be a sequence of integer points of strictly increasing norms. We fix integers $k \geq 0, p \geq 0$ and a $(k+1)$-tuple

$$
\mathbf{d}=\left(d_{0}, \ldots, d_{k}\right) \in \mathbb{Z}_{\geq 0}^{k+1} .
$$

For each $j=0, \ldots, k$, we also choose a triple of indeterminates $\mathbf{u}_{j}=\left(u_{j, 0}, u_{j, 1}, u_{j, 2}\right)$. We define the weight of a monomial

$$
\mathbf{u}_{0}^{\mathbf{e}_{0}} \cdots \mathbf{u}_{k}^{\mathbf{e}_{k}}=\prod_{j=0}^{k} \prod_{l=0}^{2} u_{j, l}^{e_{j, l}} \in \mathbb{Q}\left[\mathbf{u}_{0}, \ldots, \mathbf{u}_{k}\right]
$$

to be

$$
\sum_{j=0}^{k}\left(\sigma_{1} e_{j, 1}+\sigma_{2} e_{j, 2}\right)
$$

and its multi-degree to be

$$
\left(\left|\mathbf{e}_{0}\right|, \ldots,\left|\mathbf{e}_{k}\right|\right)=\left(e_{0,0}+e_{0,1}+e_{0,2} \ldots, e_{k, 0}+e_{k, 1}+e_{k, 2}\right) .
$$


Finally, we denote by $E(\mathbf{d}, p)$ the subspace of $\mathbb{Q}\left[\mathbf{u}_{0}, \ldots \mathbf{u}_{k}\right]$ generated by all monomials of multi-degree $\mathbf{d}$ and weight $p$. We also write

$$
\mathbf{1}=(1, \ldots, 1) \in \mathbb{Z}^{3 k+3}
$$

Proposition A.1 Suppose that there exist positive real numbers $\alpha_{0}, \ldots, \alpha_{k}, \beta_{0}, \ldots, \beta_{k}$ and an infinite set $I^{*}$ of positive integers such that, for any $i \in I^{*}$, we have

$$
\left\|\mathbf{x}_{t, j}\right\| \ll\left\|\mathbf{x}_{i}\right\|^{\alpha_{,}} \quad \text { and } \quad L\left(\mathbf{x}_{i+j}\right):=L_{\xi^{\sigma_{1}}, \xi^{\sigma_{2}}}\left(\mathbf{x}_{i+j}\right) \ll\left\|\mathbf{x}_{i}\right\|^{-\beta_{3}} \quad(j=0, \ldots, k)
$$

with implicd constants not depending on $i$. Suppose also that a polynomial $P \in E(\mathbf{d}, p)$ satisfies

$$
\left(\prod_{\jmath=0}^{k} \prod_{l=0}^{2}\left(\frac{\partial}{\partial u_{j, l}}\right)^{f_{, l \ell}} P\right)(\mathbf{1})=0
$$

for every choice of $f_{0,1}, f_{0,2}, \ldots, f_{k, 1}, f_{k, 2} \in \mathbb{Z}_{\geq 0}$ with

$$
\sum_{j=0}^{k}\left(\alpha_{j}+\beta_{j}\right)\left(f_{j, 1}+f_{j, 2}\right) \leq \sum_{j=0}^{k} \alpha_{j} d_{j} \quad \text { and } \quad f_{j .1}+f_{j, 2} \leq d_{j} \quad(j=0, \ldots, k) .
$$

Then we have

$$
P\left(\mathbf{x}_{i} \ldots, \mathbf{x}_{i+k}\right)=0
$$

for any sufficiently large $i \in I^{*}$.

Note that the conditions of the proposition provides a system of homogeneous linear equations with integer coefficients in the unknown coefficients of $P$. So, when this system has a non-zero solution (over $\mathbb{R}$ ), then it has a non-zero integer solution.

Proof: We note that

$$
\begin{aligned}
P\left(\mathbf{x}_{\imath}, \ldots, \mathbf{x}_{i+k}\right) & =\xi^{p} x_{\imath, 0}^{d_{0}} \cdots x_{i+k, 0}^{d_{k}} P\left(1, \frac{x_{i, 1}}{x_{i, 0} \xi^{\sigma_{l}}} \cdot \frac{x_{i, 2}}{x_{\imath, 0} \xi^{\sigma_{2}}}, \ldots, 1, \frac{x_{\imath+k, 1}}{x_{\imath+k, 0} \xi^{\sigma_{1}}} \cdot \frac{x_{i+k, 2}}{x_{\imath+k, 0} \xi^{\sigma_{2}}}\right) \\
& =\xi^{p} x_{i, 0}^{d_{0}} \cdots x_{i+k .0}^{d_{k}} \sum \prod_{j=0}^{k} \prod_{l=1}^{2} \frac{1}{f_{j, l}}\left(\left(\frac{x_{\imath+j, l}}{x_{i+, .0} \xi^{\sigma_{l}}}-1\right) \frac{\partial}{\partial u_{j, l}}\right)^{f_{3, l}} P(\mathbf{1})
\end{aligned}
$$


where the sum ranges over all choices of $f_{0.1}, f_{0,2}, \ldots, f_{k, 1}, f_{k 2} \in \mathbb{Z}_{\geq 0}$ with $f_{\jmath 1}+f_{J .2} \leq d_{\jmath}$ for $j=0, \ldots, k$. Since we have

$$
\begin{aligned}
& \left|x_{2,0}^{d_{0}} \cdots x_{\imath+k, 0}^{d_{k}} \prod_{\jmath=0}^{k} \prod_{l=1}^{2}\left(\frac{x_{l+\jmath . l}}{x_{l+\jmath, 0} \xi^{\sigma_{l}}}-1\right)^{f_{\jmath, l}}\right| \leq \prod_{\jmath=0}^{k}\left|x_{\imath+\jmath, 0}\right|^{d_{\jmath}-f_{\jmath, 1}-f_{3,2}} \prod_{l=1}^{2}\left(\frac{L\left(\mathbf{x}_{l+\jmath}\right)}{\left|\xi^{\sigma_{l}}\right|}\right)^{f_{j, l}} \\
& \ll \prod_{\jmath=0}^{k}\left\|\mathrm{x}_{\imath}\right\|^{\alpha_{j} d_{3}-\left(\alpha_{3}+\beta_{3}\right)\left(f_{j, 1}+f_{32}\right)} \\
& \ll\left\|\mathbf{x}_{l}\right\|^{\sum^{k=0}\left(\alpha_{1} d_{3}-\left(a_{3}+\beta_{3}\right)\left(f_{1,1}+f_{12}\right)\right)}
\end{aligned}
$$

the hypothesis implies

$$
\left|P\left(\mathbf{x}_{2} \ldots \mathbf{x}_{i+k}\right)\right| \ll\left\|\mathbf{x}_{\imath}\right\|^{-\varepsilon}
$$

for some $\varepsilon>0$ whenever $\imath \in I^{*}$. However, $P\left(\mathbf{x}_{\imath} \ldots, \mathbf{x}_{1+k-k}\right)$ is a rational number with bounded denominator. So, it must vanish for each sufficiently large $\imath \in I^{*}$. 


\section{Appendix B}

\section{Polynomials}

In this appendix. we state a result which, according to Jarnik's transference principle. is dual to Theorem 3.7, and we give a direct independent proof of it, using resultants.

Definition B.1 Let $\xi, \eta \in \mathbb{R}$. We say that a real number $\tau$ is a measure of linear approximation to $(1, \xi, \eta)$ if there exists a constant $c>0$, and arbitrarily large values of $X$. such that the inequalitics

$$
\left|x_{0}+x_{1} \xi+x_{2} \eta\right| \leq c X^{-\tau}, \quad\left|x_{1}\right| \leq X, \quad\left|x_{2}\right| \leq X
$$

have no non-zero solution $\left(x_{0}, x_{1}, x_{2}\right) \in \mathbb{Z}^{3}$. When this holds, we say that $c$ is a constant associated to the measure $\tau$.

Definition B.2 Let $\xi, \eta \in \mathbb{R}$. We say that a real number $\tau$ is a exponent of linear approximation to $(1, \xi, \eta)$ if there exists a constant $c>0$ such that the inequalitics

$$
\left|x_{0}+x_{1} \xi+x_{2} \eta\right| \leq c X^{-\tau}, \quad\left|x_{1}\right| \leq X, \quad\left|x_{2}\right| \leq X
$$

have a non-zero solution $\left(x_{0}, x_{1}, x_{2}\right) \in \mathbb{Z}^{3}$ for any sufficiently large $X$. In this case, we say that $c$ is a constant associated to the exponent $\tau$.

According to Jarník's Transference Theorem, a number $\tau$ is an exponent (respectively a measure) of linear approximation to $(1, \xi, \eta)$ if and only if $\lambda=1-1 / \tau$ is 
an exponent (respectively a measure) of simultaneous approximation to $(1, \xi, \eta)$ (see Theorem 1 of [5]).

By combining this result with our Theorem 3.7, we deduce:

Theorem B.3 Let $\xi \in \mathbb{R}$ be such that $1 . \xi, \xi^{3}$ are linearly independcnt over $\mathbb{Q}$. Then $\tau=\sqrt{3}+2$ is a measure of linear approximation to $\left(1, \xi, \xi^{3}\right)$.

Our proof below is largely inspired from [1]. We start with a definition and some preliminary results.

Definition B.4 The height $H(P)$ of a polynomual $P$ is the largest absolute value of its coefficients.

Fix real numbers $\xi$ and $\eta$ such that $1, \xi, \eta$ are linearly independent over $\mathbb{Q}$. For each real $X \geq 1$, consider the finite set of non-zero polynomials $P(T . U) \in \mathbb{Z} T+\mathbb{Z} U+\mathbb{Z}$ with $H(P) \leq X$. Since $1, \xi, \eta$ are linearly independent over $\mathbb{Q}$. there exists a non-zero polynomial $P$ from that set for which $|P(\xi, \eta)|$ is minimal. We call such a polynomial a minimal polynomial corresponding to $X$.

We note that minimal polynomials $P=a T+b U+c$ are primitive, in the sense that $\operatorname{gcd}(a, b, c)=1$. We also note that for any $X \geq 1$ there are exactly two minimal polynomials corresponding to $X$. If $P$ is one of them, then the other is $-P$.

If $P$ is a minimal polynomial for some real number $X \geq 1$, then it is minimal for $H(P)$. We can recursively construct a sequence of minimal polynomials $\left(P_{\imath}\right)_{i \geq 1} \subseteq$ $\mathbb{Z} T+\mathbb{Z} U+\mathbb{Z}$ with the following property:

if a minimal polynomial $P$ satisfies $H\left(P_{\imath}\right) \leq H(P)<H\left(P_{i+1}\right)$, then $P= \pm P_{1}$.

We say that such a sequence is a sequence of minimal polynomials associated to $(1, \xi, \eta)$.

We note that equivalently we could define such a sequence using the following property: 
if a minimal polynomial $P$ satisfies $\left|P_{2+1}(\xi, \eta)\right|<|P(\xi, \eta)| \leq\left|P_{\imath}(\xi, \eta)\right|$, then $P= \pm P_{i}$.

If $\left(P_{i}\right)_{i \geq 1}$ is a sequence of minimal polynomials associated to $(1, \xi, \eta)$, we usually write $Y_{i}:=H\left(P_{i}\right)$ and $M_{i}:=\left|P_{\imath}(\xi, \eta)\right|$. We note that the $Y_{\text {, form a strictly increasing }}$ sequence of positive integers and that the $M_{i}$ form a strictly decreasing sequence of positive real numbers. Moreover, for $i \neq j$, the polynomials $P_{\imath} . P_{j}$ are linearly independent.

Lemma B.5 Let $\xi, \eta \in \mathbb{R}$ such that $1, \xi, \eta$ are linearly independent over $\mathbb{Q}$. Let $\left(P_{i}\right)_{i \geq 1}$ be a sequence of minimal polynomials associated to $(1, \xi, \eta)$. Suppose that $\tau>1$ is an exponent of approximation and that $c>0$ is a constant associated to this exponent. Then, for each i sufficiently large, we have

$$
M_{2} \leq c\left(2 c_{0}\right)^{\tau} Y_{i+1}^{-\tau}
$$

where $c_{0}=2 \max \{1,|\xi|,|\eta|\}$

Proof: Fix some $i \geq 1$ and put $X=\left(Y_{i+1}-1\right) / c_{0}$. If $i$ sufficiently large, the hypotheses imply the existence of a non-zero polynomial $P(T, U)=x_{0}+x_{1} T+x_{2} U \in$ $\mathbb{Z} T+\mathbb{Z} U+\mathbb{Z}$ such that

$$
\max \left\{\left|x_{1}\right|,\left|x_{2}\right|\right\} \leq X \text { and }\left|x_{0}+x_{1} \xi+x_{2} \eta\right| \leq c X^{-\tau}
$$

and therefore:

$$
\begin{gathered}
\left|x_{0}\right| \leq\left|x_{1} \xi+x_{2} \eta\right|+c X^{-\tau}<c_{0} X+1=Y_{i+1} \\
\left|x_{1}\right| \leq X=\left(Y_{i+1}-1\right) / c_{0}<Y_{2+1} \\
\left|x_{2}\right| \leq X=\left(Y_{i+1}-1\right) / c_{0}<Y_{i+1}
\end{gathered}
$$

This means that $H(P)<Y_{i+1}=H\left(P_{i+1}\right)$ and so we obtain

$$
M_{i} \leq|P(\xi, \eta)| \leq c X^{-\tau}=c\left(2 c_{0}\right)^{\tau}\left(2\left(Y_{\imath+1}-1\right)\right)^{-\tau} \leq c\left(2 c_{0}\right)^{\tau} Y_{\imath+1}^{-\tau}
$$


The next two lemmas are very well known results (see [2]). In the second lemma, the notation $\operatorname{Res}(P, Q)$ stands for the Sylvester resultant of polynomials $\mathrm{P}$ and $\mathrm{Q}$ (in one variable).

Lemma B.6 Let $P . Q \in \mathbb{C}[T]$ be polynomials of degree at most 3. Then

$$
H(P Q) \asymp H(P) H(Q)
$$

Lemma B.7 Let $P, Q \in \mathbb{Z}[T]$ be non-zero polynomials with $(\operatorname{leg}(P) \leq m$ and $\operatorname{deg}(Q) \leq$ $n$. Then, for any complex number $\xi$. we have

$$
|\operatorname{Res}(P, Q)| \ll H(P)^{n-1} H(Q)^{m-1}(|P(\xi)| H(Q)+|Q(\xi)| H(P)) .
$$

From the latter results, we deduce:

Lemma B.8 Let P. $Q \in \mathbb{Z}[T]$ be non-zero polynomials of degrec at most 3 with greatest common divsor $L \in \mathbb{Z}[T]$ of degree 1. Then, for any complex number $\xi$, we have

$$
H(L)^{3}|L(\xi)| \ll H(P) H(Q)(|P(\xi)| H(Q)+|Q(\xi)| H(P)) .
$$

Proof: If $L(\xi)=0$ we are done. Suppose $L(\xi) \neq 0$. Since $P / L$ and $Q / L$ are relatively prime their resultant is a non-zero integer. From the previous lemmas, it follows that

$$
\begin{aligned}
1 \leq|\operatorname{Res}(P / L, Q / L)| & \ll H(P / L) H(Q / L)(|(P / L)(\xi)| H(Q / L)+|(Q / L)(\xi)| H(P / L)) \\
& \asymp \frac{H(P)}{H(L)} \frac{H(Q)}{H(L)}\left(\frac{|P(\xi)|}{|L(\xi)|} \frac{H(Q)}{H(L)}+\frac{|Q(\xi)|}{|L(\xi)|} \frac{H(P)}{H(L)}\right) .
\end{aligned}
$$

Identifying polynomials in $\mathbb{Z} T^{3}+\mathbb{Z} T+\mathbb{Z}$ with the triples of their coeflicients in $\mathbb{Z}^{3}$. we also note that: 
Lemma B.9 Let $P, Q, R \in \mathbb{Z} T^{3}+\mathbb{Z} T+\mathbb{Z}$. Then, for any $\xi \in \mathbb{C}$, we have

$$
|\operatorname{det}(P, Q, R)| \leq 2(|P(\xi)| H(Q) H(R)+|Q(\xi)| H(P) H(R)+|R(\xi)| H(P) H(Q))
$$

This follows by observing as in the proof of Lemma 4 of [3] that the determinant of the matrix $(P . Q, R)$ does not change if, in this matrix. we replace the constant coefficients of the polynomials by their values at $\xi$.

Lemma B.10 Let $\xi, \eta \in \mathbb{R}$ be such that $1, \xi, \eta$ are linearly independent, over $\mathbb{Q}$. Let $\left(P_{i}\right)_{i \geq 1}$ be a sequence of minimal polynomials associated to $(1 . \xi, \eta)$. Suppose that $\tau>1$ is an cxponent of approximation to $(1, \xi, \eta)$. Then there are infinitely many indices $i \geq 2$ for which $P_{i-1}, P_{\imath}, P_{i+1}$ are linearly independent over $\mathbb{Q}$.

Proof: $\quad$ For $i \geq 2$. Denote by $W_{i}$ the subspace of $\mathbb{Q}[T]$ of dimension 2 generated by $P_{i-1}$ and $P_{i}$. Suppose on the contrary that $P_{i-1}, P_{i}, P_{i+1}$ are linearly dependent over $\mathbb{Q}$ for every $i \geq i_{0}$. Then $W_{i}=W_{i+1}$ for all $i>i_{0}$ and so there exists a subspace $W$ of $\mathbb{Q}[T]$ such that $W=W_{i}$ for all $i \geq i_{0}$. Let $\{P, Q\}$ be a basis of $W \cap \mathbb{Z}[T]$. Then. for each $i \geq i_{0}$ we can write

$$
P_{i}=a_{i} P+b_{i} Q
$$

for some integers $a_{i}$ and $b_{i}$ with $\left|a_{i}\right| \ll Y_{i}$. Since $P_{\imath}$ and $P_{i+1}$ are linearly independent, we get

$$
1 \leq\left\|\begin{array}{cc}
a_{i} & b_{\imath} \\
a_{i+1} & b_{\imath+1}
\end{array}\right\|=\frac{\left|a_{i} P_{\imath+1}(\xi)-a_{i+1} P_{i}(\xi)\right|}{|Q(\xi)|} \ll Y_{i+1}\left|P_{i}(\xi)\right| \ll Y_{i+1}^{1-\tau}
$$

which is impossible for $i$ sufficiently large.

We now look at polynomials of a very specific form:

Proposition B.11 Let $P \in \mathbb{Z} T^{3}+\mathbb{Z} T+\mathbb{Z}$ be a non-zero primitive polynomial whose coefficient of $X^{2}$ is zero. Suppose that $P=\left(a T^{2}+b T+c\right)(d T+e)$ for some $a, b, c, d, e \in$ $\mathbb{Z}$. Then $a= \pm d s$ and $b=$ Fes for some $s \in \mathbb{Z}$. 
Proof: If $a=b=0$ then set $s=0$. Otherwise, set $s=\operatorname{gcd}(a, b)$. We find

$$
P=\left(a T^{2}+b \Gamma+c\right)(d T+\rho)=a d T^{3}+(a e+b d) T^{2}+(b e+c d) T+c e
$$

but $P \in \mathbb{Z} T^{3}+\mathbb{Z} T+\mathbb{Z}$ so we must have ae $+b d=0$ which implies $(a / s) e+(b / s) d=0$. Note that $\operatorname{gcd}(a / s, b / s)=1$ and that. since $P$ is primitive, we also have $\operatorname{gcd}(d, e)=1$. We conclude that $a= \pm d s$ and $b=\mp e s$.

Corollary B.12 Let $P, Q \in \mathbb{Z} T^{3}+\mathbb{Z} T+\mathbb{Z}$ be non-zero primitue polynomaals and let $L=\operatorname{gcd}(P, Q)$. Then $P= \pm Q$ or $\operatorname{deg}(L) \leq 1$.

Corollary B.13 Lct P.Q. $R \in \mathbb{Z} T^{3}+\mathbb{Z} T+\mathbb{Z}$ be non-zero premitive polynomials and let $L=\operatorname{gcd}(P . Q, R)$. If $\operatorname{deg}(L)=1$, then $P . Q, R$ are linearly dependent.

We conclude with:

Proof of Theorem B.3: Let $\left(P_{l}\right)_{1 \geq 1}$ be a sequence of minimal polynomials associated to $\left(1, \xi, \xi^{3}\right)$. Suppose on the contrary that $\tau=\sqrt{3}+2$ is not a measure of approximation. Then for every $r>0, \tau$ is an exponent of approximation.

By the previous lemmas, for each $\imath$ such that $P_{\imath-1}, P_{\imath} . P_{\imath+1}$ are linearly independent, we have

$$
\begin{aligned}
1 \leq\left|\operatorname{det}\left(P_{\imath-1}, P_{\imath}, P_{\imath+1}\right)\right| & \ll\left|P_{\imath-1}(\xi)\right| H\left(P_{\imath}\right) H\left(P_{\imath+1}\right) \\
& \ll M_{\imath-1} Y_{\imath} Y_{\imath+1} \ll c Y_{\imath}^{1-\tau} Y_{\imath+1}
\end{aligned}
$$

We also note that since $\tau>3$ this gives

$$
Y_{2}^{2} \ll Y_{i+1}
$$

Let $L=\operatorname{gcd}\left(P_{1}, P_{\imath+1}\right)$. Since $P_{\imath}$ and $P_{z+1}$ are linearly independent, by Corollary B.12 we have that $\operatorname{deg}(L) \in\{0,1\}$. Suppose $\operatorname{deg}(L)=1$. Then by Lemma B.8 we 
have

$$
\begin{aligned}
H(L)^{3}|L(\xi)| & \ll H\left(P_{\imath}\right) H\left(P_{\imath+1}\right)\left(\left|P_{\imath}(\xi)\right| H\left(P_{\imath+1}\right)+\left|P_{\imath+1}(\xi)\right| H\left(P_{\imath}\right)\right) \\
& \ll Y_{\imath} Y_{\imath+1}\left(Y_{\imath} M_{\imath+1}+Y_{\imath+1} M_{\imath}\right) \\
& \ll Y_{\imath} Y_{\imath+1}^{2-\tau} .
\end{aligned}
$$

We note that by Corollary B.13 we have $\operatorname{Res}\left(L, P_{\imath-1}\right) \neq 0$ since $P_{\imath-1}, P_{\imath}, P_{r+1}$ are linearly independent. By Lemma B.7 we also have

$$
\begin{aligned}
1 \leq\left|\operatorname{Res}\left(P_{\imath-1}, L\right)\right| & \ll H(L)^{2}\left(\left|P_{\imath-1}(\xi)\right| H(L)+|L(\xi)| H\left(P_{\imath-1}\right)\right) & & \\
& \ll M_{\imath-1} H(L)^{3}+Y_{\imath-1} H(L)^{2}|L(\xi)| & & \\
& \ll Y_{\imath}^{3-\tau}+Y_{\imath-1} H(L)^{3}|L(\xi)| & & \left(H(L) \ll H\left(P_{\imath}\right)=Y_{\imath}\right) \\
& \ll Y_{\imath}^{-3-\tau}+Y_{\imath-1} Y_{\imath} Y_{\imath+1}^{2-\tau} & & \text { (equation (B.3)) } \\
& \leq Y_{\imath}^{-3-\tau}+Y_{\imath}^{\gamma_{2}^{2} Y_{\imath+1}^{2-\tau}} & & \left(Y_{\imath-1} \leq Y_{\imath}\right) \\
& \ll Y_{\imath}^{3-\tau}+Y_{\imath+1}^{3-\tau} & & \text { (equation (B.2)) }
\end{aligned}
$$

which is a contradiction for $i$ sufficiently large since $\tau>3$.

Therefore. we may assume that $\operatorname{deg}(L)=0$, and so, applying Lemma B.7. we get

$$
\begin{aligned}
1 & \leq\left|\operatorname{Res}\left(P_{\imath}, P_{\imath+1}\right)\right| \\
& \ll H\left(P_{\imath}\right)^{2} H\left(P_{\imath+1}\right)^{2}\left(H\left(P_{\imath+1}\left|P_{\imath}(\xi)\right|+H\left(P_{\imath}\right)\left|P_{\imath+1}(\xi)\right|\right)\right. \\
& \ll Y_{\imath}^{2} Y_{\imath+1}^{2}\left(Y_{\imath+1} M_{\imath}+Y_{\imath} M_{\imath+1}\right) \\
& \ll c Y_{\imath}^{2} Y_{\imath+1}^{3-\tau} .
\end{aligned}
$$

Combining the estimates (B.1) and (B.4). we conclude that, for each $\imath$ such that $P_{\imath-1}, P_{\imath} . P_{\imath+1}$ are linearly independent, we have

$$
1 \ll c^{\tau-1} Y_{\imath}^{-2(\tau-1)} Y_{\imath+1}^{-\tau^{2}+4 \tau-3} \ll c^{1+\tau} Y_{\imath+1}^{-\tau^{2}+4 \tau-1}
$$

This occurs for infinitely many $i$ (by Lemma B.10), but $\tau^{2}-4 \tau+1=0$ and so, for a c sufficiently small. this is impossible. 


\section{Bibliography}

[1] B. Arbour, D. Roy, A Gel'fond type criterion in degree two. Acta Arith. 111 (2004). 97-103.

[2] W. D. Brownawell, Sequences of diophantine approximations. J. Number Theory $6(1974), 11-21$.

[3] H. Davenport, II. M. Schmidt, Approximation to real numbers by quadratic irrationals, Acta Arth. 13 (1967), 169-176.

[4] H. Davenport, W. M. Schmidt. Approximation to real numbers by algebraic integers. Acta Arth. 15 (1969). 393-416.

[5] V. Jarník. Zum Khintchineschen Übertragungssatz, Trudy Tbllzsskogo mathematıcheskogo instıtuta 2 m. A. M. Razmadze = Travaux de l'Instıtut mathématique de Tbrlisst 3 (1938), 193-212.

[6] W. Nef, Linear Algebru, Dover, 1988.

[7] D. Rov, $\Lambda$ pproximation to real numbers by cubic algebraic integers I, Proc. London Math. Soc. 88 (2004). 42-62.

[8] D. Roy: Approximation to real numbers by cubic algebraic integers II, Annals of Mathematics 158 (2003). 10811087. 
[9] D. Roy, Diophantine approximation in small degree, Number Theory, CRM Proceedings and Leciure Notes 36 (2004), 269-285.

[10] W. M. Schmidt, Diophantine approximation, Lecture Notes in Math., vol. 785, Springer-Verlag, 1980.

[11] D. Zelo. Simultaneous Approximation to Real and p-adic Numbers, Ph.D. Thesis. University of Ottawa, 2008. 